Peter Gerlinger

Multi-dimensional limiting for high-order schemes including turbulence and combustion

Journal of Computational Physics 231 (5), 2199-2228, 2012.

The original publication is available at www.elsevier.com

http://dx.doi.org/10.1016/j.jcp.2011.10.024 


\title{
Multi-dimensional limiting for high-order schemes including turbulence and combustion
}

\author{
Peter Gerlinger \\ Institute of Combustion Technology of Aerospace Engineering, University of Stuttgart, \\ Pfaffenwaldring 38-40, 70569 Stuttgart, Germany
}

\begin{abstract}
In the present paper a fourth/fifth order upwind biased limiting strategy is presented for the simulation of turbulent flows and combustion. Because high order numerical schemes usually suffer from stability problems and TVD approaches often prevent convergence to machine accuracy the multi-dimensional limiting process (MLP) [1] is employed. MLP uses information from diagonal volumes of a discretization stencil. It interacts with the TVD limiter in such a way, that local extrema at the corner points of the volume are avoided. This stabilizes the numerical scheme and enables convergence in cases, where standard limiters fail to converge. Up to now MLP has been used for inviscid and laminar flows only. In the present paper this technique is applied to fully turbulent sub- and supersonic flows simulated with a low Reynolds-number turbulence closure. Additionally, combustion based on finite-rate chemistry is investigated. An improved MLP version (MLP ${ }^{l d}$, low diffusion) as well as an analysis of its capabilities and limitations are given. It is demonstrated, that the scheme offers high accuracy and robustness while keeping the computational cost low. Both steady and unsteady test cases are investigated.
\end{abstract}

Keywords: higher order spatial discretization, multi-dimensional limiting process, shock resolution, TVD condition, compressible flow, combustion, turbulence model

Email address: peter.gerlinger@dlr.de ()

Preprint submitted to Journal of Computational Physics

November 9, 2011 


\section{Introduction}

The discretization of inviscid fluxes is still a challenging part of numerical simulation. Especially in supersonic flow there is a demand for high accuracy discretizations which suppress oscillations at shock waves and maintain monotonicity. In classical high order MUSCL (Monotonic Upstream-centered Scheme for Conservation Laws) [2] approaches, TVD (Total Variation Diminishing) limiters [3] are used to avoid oscillations at discontinuities. Besides classical TVD limiters like minmod, superbee, van Albada, or the van Leer limiter, there is a number of newly developed limiter functions $[4,5]$ which are constructed to achieve a sharp and accurate shock capturing while at the same time avoid clipping and squaring effects of classical second order limiters [5]. Some limiter functions are even able to maintain their formal accuracy at local extrema [5]. Another topic of research is the handling of interface value reconstruction on highly stretched irregular grids. Moreover, there are activities to use multi-dimensional information in the limiter design. Conventional flux vector or flux difference splittings treat any coordinate direction separately from the remaining ones. It is easy to show, that such one-dimensional limiters fail to achieve a good shock resolution if the shock is located in direction diagonal to the computational grid. This may cause an oscillatory behavior and a stall of convergence. During the last two decades there was some activity in developing multi-dimensional limiting techniques $[6,7,8]$ without meeting a wide acceptance. However, the newly developed MLP approach of Kim and coworkers $[9,1,10,11]$ seems to have a high potential to achieve significant improvements in this field.

An alternative to these discretization techniques are ENO/WENO [12, 13, 14] schemes, which use a number of stencils from which the smoothest ones are chosen. The great advantage of WENO schemes in supersonic flow is the ability to achieve high order accuracies at discontinuities. However, the associated numerical effort is relatively high, especially if the interface values are calculated from characteristic variables. Some authors report this to be necessary to avoid 
numerical oscillations [15]. Moreover, convergence problems may appear in case of steady state problems.

In the present paper the multi-dimensional limiting process (MLP) of Kim et al. $[9,1]$ is combined with the $\mathrm{AUSM}^{+}$-up flux vector splitting of Liou [16]. The combination of both approaches promises a good monotone shock capturing and a good convergence behavior at relatively low computational cost. The disadvantage of the present scheme is, that due to the limiter function which avoids oscillations at shock waves, the discretization order may be reduced to first order locally. MLP shares this property with conventional TVD limiters. On the other hand, there is a low computational effort and a high numerical stability. In this paper the new discretization technique is extended to nonequal grid spacing for cell interface interpolation functions up to fifth order. Some improvements in comparison to the standard MLP [1] will be given and a modified MLP version, MLP ${ }^{l d}$ (low diffusion), will be presented. The new approach is used for simulations including turbulence and combustion. This is an extension to the work of Kim et al. [9, 1] who treated laminar and inviscid flows only.

\section{Governing equations and numerical scheme}

For the investigation of high speed turbulent combustion the averaged expanded Navier-Stokes, turbulence, and species transport equations are solved, which are given by

$$
\frac{\partial \mathbf{Q}}{\partial t}+\frac{\partial\left(\mathbf{F}-\mathbf{F}_{\nu}\right)}{\partial x}+\frac{\partial\left(\mathbf{G}-\mathbf{G}_{\nu}\right)}{\partial y}+\frac{\partial\left(\mathbf{H}-\mathbf{H}_{\nu}\right)}{\partial z}=\mathbf{S} .
$$

The vector of conservative variables is

$$
\mathbf{Q}=\left[\bar{\rho}, \bar{\rho} \widetilde{u}, \bar{\rho} \widetilde{v}, \bar{\rho} \widetilde{w}, \bar{\rho} \widetilde{E}, \bar{\rho} q, \bar{\rho} \omega, \bar{\rho} \widetilde{Y}_{\alpha}\right]^{T}, \quad \alpha=1,2, \ldots, N_{k}-1,
$$

where $\mathbf{F}, \mathbf{G}$, and $\mathbf{H}$ are inviscid, and $\mathbf{F}_{\nu}, \mathbf{G}_{\nu}$, and $\mathbf{H}_{\nu}$ are viscous fluxes in $x$-, $y$ - and $z$-direction, respectively. The variables in Eq. (2) are the averaged

density $\bar{\rho}$, the velocity components $\widetilde{u}, \widetilde{v}$, and $\widetilde{w}$, the total specific energy $\widetilde{E}$, 
the turbulence variables $q=\sqrt{k}$ and $\omega$ ( $k$ is the turbulent kinetic energy and $\omega=\epsilon / k, \epsilon$ is the dissipation rate of $k$ ), and the species mass fractions $\widetilde{Y}_{\alpha} . N_{k}$ denotes the number of different species. For turbulence closure a two-equation low-Reynolds-number $q-\omega$ turbulence model is employed [17]. Contributions to the source vector

$$
\mathbf{S}=\left[0,0,0,0,0, S_{q}, S_{\omega}, S_{\alpha}\right]^{T}, \quad \alpha=1,2, \ldots, N_{k}-1
$$

arise from the $q$ and $\omega$ and the species transport equations. The species source terms are given by

$$
S_{\alpha}=M_{\alpha} \sum_{r=1}^{N_{r}}\left[\left(\nu_{\alpha, r}^{\prime \prime}-\nu_{\alpha, r}^{\prime}\right)\left(k_{f_{r}} \prod_{l=1}^{N_{k}} c_{l}^{\nu_{l, r}^{\prime}}-k_{b_{r}} \prod_{l=1}^{N_{k}} c_{l}^{\nu_{l, r}^{\prime \prime}}\right)\right],
$$

where $k_{f_{r}}$ and $k_{b_{r}}$ are the forward and backward reaction rates of reaction $r$, $N_{r}$ is the number of reactions, and $c_{l}$ the concentration of species $l$.

The unsteady governing equations are integrated in time using an implicit finite-volume LU-SGS (Lower-Upper Symmetric Gauß-Seidel) algorithm [18, 19]. Beside the source term and the inviscid flux Jacobians, simplified viscous flux Jacobians based on the thin-layer Navier-Stokes equations are included in the implicit part of the numerical solver. The following set of equations has to be solved at any time step

$$
\mathcal{L}(\mathbf{Q}) \Delta \mathbf{Q}=\mathbf{R}(\mathbf{Q})
$$

where $\mathcal{L}$ is an implicit operator including the Jacobians, $\mathbf{R}$ is the residual, and $\Delta \mathbf{Q}$ is the correction of the variable vector. The linearized set of equations is factorized and solved in two subsequent steps by a lower and upper sweep through the computational domain $[19,20]$. While a first order temporal discretization is used for all steady state problems, a second or third order BDF (backward differentiation formula) scheme with subiterations is chosen for time-accurate calculations. Varying time-step sizes are taken into account in the BDF coefficients. The non-linear set of equations is solved at any time-step by a number of inner Newton iterations (dual time stepping) until the residual is dropped up to a predefined limit. 


\section{Multidimensional limiting process}

In a number of papers Kim et al. [9, 1, 10, 21, 11] developed the multidimensional limiting process (MLP) for different applications. In contrast to conventional TVD schemes, MLP uses multi-dimensional information for slope limitation. This enables an efficient and monotonic numerical scheme and prevents oscillations across discontinuities which are oblique to the computational grid. MLP may be seen as an extension to conventional second order limiters such as minmod, van Leer, or superbee by considering information from diagonal volumes. Besides an improvement of the numerical solution it additionally stabilizes the numerical scheme as shown by Kim et al. $[9,1]$. However, the great potential of MLP is its easy application to higher order spatial interpolation (fourth, fifth order or higher). Such high order schemes may be used for compressible supersonic flows while maintaining numerical stability and convergence.

From the above cited papers concerning MLP there is only one [1] which treats three-dimensional flows. This version differs from the other ones in that geometrical considerations, by using gradient angles for flow variables, are avoided. Such a treatment seems to be not practical in 3D. Because the present analysis and the proposed extensions of MLP are intended to work in both 2D and 3D, the 3D version of MLP is taken as a basis. While presenting MLP, a different approach is used than in the original paper of Kim et al. [1]. This offers another view, from which some corrections and improvements are derived.

\subsection{One-dimensional reconstruction of unlimited interface values}

In the framework of cell centered finite-volume discretization, inviscid fluxes have to be calculated at the cell interfaces. This requires interface values, which in the present case, are the primitive variables $\rho, u, v, w, H, q, \omega, Y_{\alpha}$, and $\bar{\gamma} . H$ is the total enthalpy and $\bar{\gamma}$ the integral specific heat ratio which is required to calculate the critical speed of sound $a^{*}$ in the $\mathrm{AUSM}^{+}$-up flux vector splitting. The interface values are reconstructed from cell centered values with a spatial accuracy of up to fifth order. 


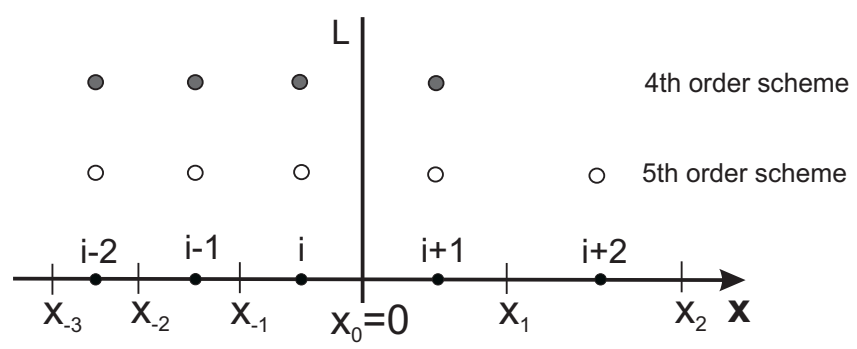

Figure 1: Sketch of the discretization stencils for the one-dimensional fourth and fifth order upwind biased calculation of the left $(L)$ interface state at $i+1 / 2(x=0)$. The circles indicate the cell averages used.

Piecewise second or third order left $(L)$ and right $(R)$ interface states are obtained from the MUSCL approach [2] by

$$
\begin{aligned}
& q_{i+1 / 2}^{L}=q_{i}+\frac{1}{4}\left[(1-\kappa) \Delta q_{i-1 / 2}+(1+\kappa) \Delta q_{i+1 / 2}\right], \\
& q_{i+1 / 2}^{R}=q_{i+1}-\frac{1}{4}\left[(1-\kappa) \Delta q_{i+3 / 2}+(1+\kappa) \Delta q_{i+1 / 2}\right],
\end{aligned}
$$

where $q_{i}$ is an averaged value for volume $i$ and $\kappa$ is a free parameter to obtain different second order and a third order $(\kappa=1 / 3)$ scheme. The cell interface values of Eq. (6) may be interpreted as a first order upwind representation plus an additional anti-diffusive term. The higher order corrections depend on local gradients (e.g. $\Delta q_{i-1 / 2}=q_{i}-q_{i-1}$ ), one across the cell face in question and the second immediately upwind [22].

Fourth and fifth order schemes are derived by a polynomial reconstruction $[12,1]$. If $x=0$ is located at the interface $i+1 / 2$ (see Fig. 1 ), the polynomials

$$
\begin{aligned}
& \hat{q}(x)=A x^{3}+B x^{2}+C x+D, \\
& \hat{q}(x)=A x^{4}+B x^{3}+C x^{2}+D x+E
\end{aligned}
$$

are used to calculate the left interface state $q_{i+1 / 2}^{L}$ with fourth or fifth order accuracy, respectively. During discretization the difference between the $i+1 / 2$ and $i-1 / 2$ fluxes is taken, and a fourth or fifth order scheme is obtained, respectively. The coefficients $(A, B, \ldots, E)$ follow from a number of cell- 
averages

$$
q_{i+m}=\frac{1}{\Delta x_{i}} \int_{x_{i+m-1 / 2}}^{x_{i+m+1 / 2}} \hat{q}(x) d x
$$

with $m=-2,-1, \ldots, 1$ for the fourth order scheme, and $m=-2,-1, \ldots, 2$ for the fifth order scheme, respectively. The chosen discretization stencils for the fourth and fifth order upwind biased determination of $q_{i+1 / 2}^{L}$ (used in this paper) are shown in Fig. 1. Right interface values are calculated correspondingly. Based on cell averages, the unlimited left and right interface states at $i+1 / 2$ follow from

$$
\begin{aligned}
& q_{i+1 / 2}^{L}=a_{1}^{L} q_{i-2}+a_{2}^{L} q_{i-1}+a_{3}^{L} q_{i}+a_{4}^{L} q_{i+1}, \\
& q_{i+1 / 2}^{R}=a_{1}^{R} q_{i}+a_{2}^{R} q_{i+1}+a_{3}^{R} q_{i+2}+a_{4}^{R} q_{i+3},
\end{aligned}
$$
4 th order 0$)$

and

$$
\begin{aligned}
& q_{i+1 / 2}^{L}=b_{1}^{L} q_{i-2}+b_{2}^{L} q_{i-1}+b_{3}^{L} q_{i}+b_{4}^{L} q_{i+1}+b_{5}^{L} q_{i+2} \\
& q_{i+1 / 2}^{R}=b_{1}^{R} q_{i-1}+b_{2}^{R} q_{i}+b_{3}^{R} q_{i+1}+b_{4}^{R} q_{i+2}+b_{5}^{R} q_{i+3}
\end{aligned}
$$

The chosen stencils use three upwind and one downwind point for the fourth order scheme and three upwind and two downwind points in case of the fifth order scheme, respectively. Thus, the fourth order scheme has a stronger upwind character which, at least for one supersonic test case, was found to be advantageous. Both schemes require three values at each side of the interface. With respect to parallelization by domain decomposition, three rows of ghost volumes have to be exchanged between adjacent blocks, located on different CPUs. For every cell interface left and right coefficients $\left(a_{i}^{L, R}\right.$ or $\left.b_{i}^{L, R}\right)$ have to be calculated. Non-equal grid spacing is taken into account. TVD and MLP limitations (which follow in the next section) are usually based on slope ratios

$$
r_{i}^{L}=\Delta q_{i+1 / 2} / \Delta q_{i-1 / 2}, \quad r_{i}^{R}=\Delta q_{i-1 / 2} / \Delta q_{i+1 / 2}
$$

which are connected by $r_{i}^{R}=1 / r_{i}^{L}$. Note that these ratios are formed by the gradient across the cell interface in question $(i+1 / 2$ in case of $L$ and $i-1 / 2$ in case of $R$ ) and the gradient immediately upwind $(i-1 / 2$ in case of $L$ and 
Table 1: Dependency of $\beta_{i}^{L}$ and $\beta_{i+1}^{R}$ from slope ratios $r$ for different orders of accuracy

\begin{tabular}{ccc}
\hline$\beta_{i}^{L}$ & $\beta_{i+1}^{R}$ & order of accuracy \\
\hline$\beta_{i}^{L}\left(r_{i}^{L}\right)$ & $\beta_{i+1}^{R}\left(r_{i+1}^{R}\right)$ & 2nd or 3rd \\
$\beta_{i}^{L}\left(r_{i-1}^{L}, r_{i}^{L}\right)$ & $\beta_{i+1}^{R}\left(r_{i+1}^{R}, r_{i+2}^{R}\right)$ & 4 th \\
$\beta_{i}^{L}\left(r_{i-1}^{L}, r_{i}^{L}, r_{i+1}^{L}\right)$ & $\beta_{i+1}^{R}\left(r_{i}^{R}, r_{i+1}^{R}, r_{i+2}^{R}\right)$ & 5 th \\
\hline
\end{tabular}

$i+1 / 2$ in case of $R$ ). Equations (10) and (11) can be rewritten as functions of $r_{i}$

$q_{i+1 / 2}^{L}=q_{i}+0.5\left(c_{1}^{L} / r_{i-1}^{L}+c_{2}^{L}+c_{3}^{L} r_{i}^{L}\right) \Delta q_{i-1 / 2}$,

$q_{i+1 / 2}^{R}=q_{i+1}-0.5\left(c_{1}^{R} r_{i+1}^{R}+c_{2}^{R}+c_{3}^{R} / r_{i+2}^{R}\right) \Delta q_{i+3 / 2}$

4 th or(de3)

and

$q_{i+1 / 2}^{L}=q_{i}+0.5\left(d_{1}^{L} / r_{i-1}^{L}+d_{2}^{L}+d_{3}^{L} r_{i}^{L}+d_{4}^{L} r_{i}^{L} r_{i+1}^{L}\right) \Delta q_{i-1 / 2} ，$

$q_{i+1 / 2}^{R}=q_{i+1}-0.5\left(d_{1}^{R} r_{i}^{R} r_{i+1}^{R}+d_{2}^{R} r_{i+1}^{R}+d_{3}^{R}+d_{4}^{R} r_{i+2}^{R}\right) \Delta q_{i+3 / 2}$

5 th or(dles)

As before, the coefficients of the polynomial functions $c_{i}^{L, R}$ or $d_{i}^{L, R}$ depend on the cell metrics only. The second and third order MUSCL approach from Eq. (6) may be expressed as a function of the corresponding upwind gradient too, and

$$
\begin{aligned}
& q_{i+1 / 2}^{L}=q_{i}+\frac{1}{4}\left[(1-\kappa)+(1+\kappa) r_{i}^{L}\right] \Delta q_{i-1 / 2} \\
& q_{i+1 / 2}^{R}=q_{i+1}-\frac{1}{4}\left[(1-\kappa)+(1+\kappa) r_{i+1}^{R}\right] \Delta q_{i+3 / 2}
\end{aligned}
$$

is obtained. By introduction of the parameters $\beta_{i}^{L}$ and $\beta_{i+1}^{R}$ the MUSCL approach and the higher order reconstructions from Eqs. (13) and (14) are combined in one equation [1]

$$
\begin{aligned}
& q_{i+1 / 2}^{L}=q_{i}+0.5 \beta_{i}^{L} \Delta q_{i-1 / 2}, \\
& q_{i+1 / 2}^{R}=q_{i+1}-0.5 \beta_{i+1}^{R} \Delta q_{i+3 / 2} .
\end{aligned}
$$


Table 2: Functions $\beta_{i}^{L}$ and $\beta_{i+1}^{R}$ for different orders of accuracy in case of an equal grid spacing. The various schemes are the 2nd order linear-upwind ( $\mathrm{U}, \kappa=-1)$, Fromm (Fr, $\kappa=0)$, central difference $(\mathrm{CD}, \kappa=1)$, the 3 rd order cubic $(\kappa=1 / 3)$, and a 4 th and a 5 th order upwind biased (Ub) scheme.

\begin{tabular}{ccc}
\hline$\beta_{i}^{L}$ & $\beta_{i+1}^{R}$ & order of accuracy \\
\hline 1 & 1 & 2 nd $\mathrm{U}$ \\
$\left(1+r_{i}^{L}\right) / 2$ & $\left(1+r_{i+1}^{R}\right) / 2$ & $2 \mathrm{nd} \mathrm{Fr}$ \\
$r_{i}^{L}$ & $r_{i+1}^{R}$ & $2 \mathrm{nd} \mathrm{CD}$ \\
$\left(1+2 r_{i}^{L}\right) / 3$ & $\left(1+2 r_{i+1}^{R}\right) / 3$ & $3 \mathrm{rd} \mathrm{Ub}$ \\
$\left(-1 / r_{i-1}^{L}+4+3 r_{i}^{L}\right) / 6$ & $\left(3 r_{i+1}^{R}+4-1 / r_{i+2}^{R}\right) / 6$ & 4 th Ub \\
$\left(-2 / r_{i-1}^{L}+11+24 r_{i}^{L}-3 r_{i}^{L} r_{i+1}^{L}\right) / 30$ & $\left(-3 r_{i}^{R} r_{i+1}^{R}+24 r_{i+1}^{R}+11-2 / r_{i+2}^{R}\right) / 30$ & 5 th Ub \\
\hline
\end{tabular}

Table 1 shows which and how many slope ratios $r$ are required for a $\beta_{i}^{L}$ and $\beta_{i+1}^{R}$ of a certain spatial accuracy. The coefficients $c_{i}^{L, R}$ or $d_{i}^{L, R}$ have to be determined in advance for any interface and coordinate direction, if non-equal grid spacing is taken into account. This is done in the present paper for the fourth and fifth order schemes. In case of an equally spaced grid (or if a non-equal grid spacing is neglected) these locally varying coefficient become constants and are identical for any cell interface. Table 2 summarizes $\beta_{i}^{L}$ and $\beta_{i+1}^{R}$ functions for different orders of accuracy in case of an equally spaced grid. The first four interpolation schemes result from the MUSCL approach while using different values of $\kappa$. For $\kappa=-1$ a second order fully upwind scheme is obtained. Any other value introduces more or less downwind influence. The Fromm scheme $(\kappa=0)$ and the central difference scheme $(\kappa=1)$ are of second order accuracy. The first one has been designed to minimize dispersion errors [22]. The only third order MUSCL scheme (cubic-upwind interpolation) requires $\kappa=1 / 3$. Finally, interface value reconstructions for the upwind biased fourth and fifth order schemes described before are listed in the last two lines of Table 2. 


\subsection{MLP reconstruction of interface values}

It is easy to show for scalar problems, that the unlimited interface value reconstructions given in Table 2 are not able to achieve a well resolved, nonoscillatory discontinuity. Thus the basic idea of TVD methods is to increase the amount of numerical dissipation at discontinuities, while maintaining high order accuracy in smooth regions. To do this, a TVD limiter $\Phi$ is introduced and Eq. (16) is replaced by

$$
\begin{aligned}
& q_{i+1 / 2}^{L}=q_{i}+0.5 \Phi\left(r^{L}\right) \Delta q_{i-1 / 2}, \\
& q_{i+1 / 2}^{R}=q_{i+1}-0.5 \Phi\left(r^{R}\right) \Delta q_{i+3 / 2} .
\end{aligned}
$$

The TVD limiter depends on the ratio of up- and downstream gradients $r_{i}$, which are a measure for the local smoothness of $q$. If the limiter is symmetric $(\Phi(r)=r \Phi(1 / r))$, than Eq. (17) directly follows from the MUSCL approach which becomes independent of $\kappa$. According to Sweby [23] the one-dimensional scalar TVD constraint is given by $0 \leq \Phi(r) \leq \min (2 r, 2)$. Due to $\Phi(r)=0$ for $r<0$ the limiter switches to first order accuracy if $r$ becomes negative. This is the case for any extreme point. For the third and higher order reconstructions, Kim et al. [9] use a filtering of the unlimited values by the TVD constraint of Sweby [23]

$$
\begin{aligned}
& q_{i+1 / 2}^{L}=q_{i}+0.5 \max \left[0, \min \left(2,2 r_{i}^{L}, \beta_{i}^{L}\right)\right] \Delta q_{i-1 / 2}, \\
& q_{i+1 / 2}^{R}=q_{i+1}-0.5 \max \left[0, \min \left(2,2 r_{i+1}^{R}, \beta_{i+1}^{R}\right)\right] \Delta q_{i+3 / 2} .
\end{aligned}
$$

The interface values of the second order schemes given in Table 2 (upper three lines) usually are not limited in this way. Instead a large number of limiter functions have been developed which automatically are in the second order TVD region. Corresponding values for the minmod, van Leer, and superbee limiter are

$$
\begin{array}{lr}
\Phi\left(r_{i}^{L}\right)=\beta_{i}^{L}=\min \left(1, r_{i}^{L}\right) & \text { 2nd order minmo(19) } \\
\Phi\left(r_{i}^{L}\right)=\beta_{i}^{L}=\max \left[\min \left(1,2 r_{i}^{L}\right), \min \left(2, r_{i}^{L}\right)\right] & \text { 2nd order super(2e\&) } \\
\Phi\left(r_{i}^{L}\right)=\beta_{i}^{L}=2 r_{i}^{L} /\left(1+r_{i}^{L}\right) & \text { 2rd order van Leф®,1) }
\end{array}
$$


which all have $\Phi(r)=0$ for $r<0$ in common. The TVD constraint of Sweby is the starting point for the development of MLP by Kim et al. $[9,1]$. The MPL region $\Phi_{M P L}(r)$ is defined by

$$
\begin{array}{rlrl}
\Phi_{M P L}(r) & =0 & & : r<0, \\
r \leq \Phi_{M P L}(r) \leq 2 r & & : 0 \leq r \leq 1, \\
1 \leq \Phi_{M P L}(r) \leq 2 & & : 1 \leq r,
\end{array}
$$

to be a subset of the TVD region. This definition enables the introduction a single parameter $1 \leq \alpha \leq 2$ which, in dependence of $r$, performs a linear scaling from the the upper limit $(\alpha=2)$ to the more viscous lower limit $(\alpha=1)$. TVD and MLP regions are shown in Fig. 2. The upper limit is exactly two times the minmod limiter and corresponds to the TVD region defined by Sweby [23], while at the lower limit the minmod limiter is obtained. Instead of Eq. (18) MLP uses

$$
\begin{aligned}
& q_{i+1 / 2}^{L}=q_{i}+0.5 \max \left[0, \min \left(\alpha^{L}, \alpha^{L} r_{i}^{L}, \beta_{i}^{L}\right)\right] \Delta q_{i-1 / 2}, \\
& q_{i+1 / 2}^{R}=q_{i+1}-0.5 \max \left[0, \min \left(\alpha^{R}, \alpha^{R} r_{i+1}^{R}, \beta_{i+1}^{R}\right)\right] \Delta q_{i+3 / 2}
\end{aligned}
$$

to reconstruct the interface values. As will be discussed later, values of $\alpha<2$ are used only, if the variable interpolation based on the upper MLP limit $(\alpha=2)$ causes a local extremum at one of the corners of the volume. Moreover, a limitation of $\alpha$ to $\alpha \in[1,2]$ while fulfilling the MLP condition (as demanded by

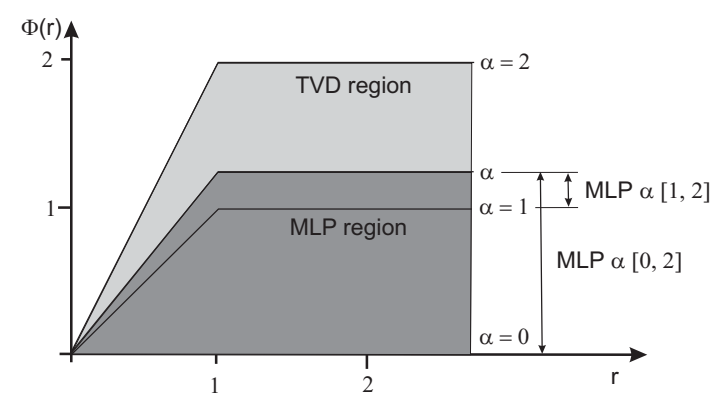

Figure 2: TVD region of Sweby [23] and MLP region of Kim et al. [9] ( $\alpha=2$ corresponds to the TVD region and $\alpha=1$ is the minmod limiter). Depending on the definition of $\alpha(\alpha \in[1,2]$ or $\alpha \in[0,2]$ different MLP regions are obtained. 
Kim et al. [9]) is possible in two-dimensional flows only. As will be shown later at least $\alpha \in[2 / 3,2]$ is required for a three-dimensional flow, while for practical reasons even $\alpha \in[0,2]$ may be used (see Fig. 2). A reduction of $\alpha$ to $\alpha=0$ is a blend to first order spatial accuracy, while $\alpha=1$ still maintains second order accuracy. Equation (23) may be used in combination with any of the second order limiters from Eqs. (19) to (21) or the third to fifth order scheme $\beta$ values from Table 2.

\section{Two-dimensional MLP}

As shown in detail in the original papers of Kim et al. [9, 1] MLP satisfies the discrete maximum principle for multi-dimensional scalar conservation laws

$$
q_{n b}^{\min } \leq q_{i, j}^{n+1} \leq q_{n b}^{\max }
$$

where $q_{n b}^{\min }$ and $q_{n b}^{\max }$ are minimum and maximum values out of some neighbouring cells. One main difference to the earlier approaches of Spekreijse [24] and Barth [25] is the choice of the neighbouring volumes. While on structured two-dimensional grids Spekreijse and Barth use the volume in question and its four neighbours (two in both coordinate directions), Kim et al. use the volume in question and all eight surrounding cells, including the four diagonal ones. This is advantageous for oscillation control in case of multi-dimensional oblique discontinuities.

The basic point of the MLP approach is to determine the parameters $\alpha$ of Eq. (23) in such a way, that the interface value reconstruction does not cause a local extremum at any of the four corners of the two-dimensional volume. The limiting condition (24) is applied to the four vertex points $\left(i+\kappa_{x} / 2, j+\kappa_{y} / 2\right)$, $\kappa_{x}, \kappa_{y}= \pm 1$ of volume $i, j$ by

$$
Q_{i+\kappa_{x} / 2, j+\kappa_{y} / 2}^{\min } \leq q_{i+\kappa_{x} / 2, j+\kappa_{y} / 2} \leq Q_{i+\kappa_{x} / 2, j+\kappa_{y} / 2}^{\max }
$$

where

$$
\begin{aligned}
& Q_{i+\kappa_{x} / 2, j+\kappa_{y} / 2}^{\min }=\min \left(q_{i, j}, q_{i+\kappa_{x}, j}, q_{i, j+\kappa_{y}}, q_{i+\kappa_{x}, j+\kappa_{y}}\right), \\
& Q_{i+\kappa_{x} / 2, j+\kappa_{y} / 2}^{\max }=\max \left(q_{i, j}, q_{i+\kappa_{x}, j}, q_{i, j+\kappa_{y}}, q_{i+\kappa_{x}, j+\kappa_{y}}\right)
\end{aligned}
$$


are taken to be the minimum and maximum cell center values surrounding a cell corner, respectively [1]. As will be shown later, the corner values $q_{i+\kappa_{x} / 2, j+\kappa_{y} / 2}$ are calculated from the interface values scaled by $\alpha$. Thus the parameter $\alpha$ can be used to satisfy condition (25). If the interface values are calculated on basis of MLP only $\left(\beta^{L}=\beta^{R}=2\right)$, Eq. (23) simplifies to

$$
\begin{aligned}
q_{i+1 / 2}^{L} & =q_{i}+0.5 \alpha^{L} \max \left[0, \min \left(1, r_{i}^{L}\right)\right] \Delta q_{i-1 / 2}, \\
q_{i+1 / 2}^{R} & =q_{i+1}-0.5 \alpha^{R} \max \left[0, \min \left(1, r_{i+1}^{R}\right)\right] \Delta q_{i+3 / 2} .
\end{aligned}
$$

Because MLP is a linear multiple of the minmod limiter, it is symmetric $(\Phi(r) / r=$ $\Phi(1 / r))$. This allows the definition of a single $\alpha=\alpha_{i}=\alpha_{i+1 / 2}^{L}=\alpha_{i-1 / 2}^{R}$ for any volume $i$ (in one flow direction). With $r_{i}=r_{i}^{L}=1 / r_{i}^{R}$ it follows that

$$
\begin{aligned}
& q_{i+1 / 2}^{L}=q_{i}+0.5 \alpha \max \left[0, \min \left(1, r_{i}\right)\right] \Delta q_{i-1 / 2}=q_{i}+0.5 \alpha \Delta q^{m m}=q_{i}+0.5 \Delta q^{M L P}, \\
& q_{i-1 / 2}^{R}=q_{i}-0.5 \alpha \max \left[0, \min \left(1, r_{i}\right)\right] \Delta q_{i-1 / 2}=q_{i}-0.5 \alpha \Delta q^{m m}=q_{i}-0.5 \Delta q^{M L P},
\end{aligned}
$$

which simply shows the symmetry of the limiter. The newly defined term $\Delta q^{m m}$ is the interface correction obtained by the minmod limiter (see also Eq. (19)), and $\Delta q^{M L P}$ is the MLP correction which has to fulfill condition (25). Assuming a linear distribution, the four cell corner MLP values are calculated from the MLP reconstructed interface values by

$$
q_{i+\kappa_{x} / 2, j+\kappa_{y} / 2}^{M L P}=q_{i, j}+0.5 \kappa_{x} \alpha_{x} \Delta q_{x}^{m m}+0.5 \kappa_{y} \alpha_{y} \Delta q_{y}^{m m},
$$

with $\kappa_{x}, \kappa_{y}= \pm 1$. In contrast to Kim et al. [1], different values $\alpha_{x}$ and $\alpha_{y}$ are introduced here for both coordinate directions. Kim et al. use a single value in their derivation of MLP, and later on approximate one term instead of doing an accurate simulation. As will be shown later, this approximation has the same effect causing different $\alpha$ values for the different coordinate directions. Because the present formulation seems to be more straight forward, it is preferred in this work. This point will be discussed later in more detail. The four newly defined and MLP limited cell corner values $q_{i+\kappa_{x} / 2, j+\kappa_{y} / 2}^{M L P}$ have to fulfill condition (25)

$$
Q_{i+\kappa_{x} / 2, j+\kappa_{y} / 2}^{\min } \leq q_{i+\kappa_{x} / 2, j+\kappa_{y} / 2}^{M L P} \leq Q_{i+\kappa_{x} / 2, j+\kappa_{y} / 2}^{\max }
$$


from which $\alpha_{x}$ and $\alpha_{y}$ will be derived. In principle, all four corners of a volume must be checked whether for $\alpha_{x}=\alpha_{y}=2$ (the upper limit of the MLP region) the MLP reconstruction $q_{i+\kappa_{x} / 2, j+\kappa_{y} / 2}^{M L P}$ is a local extremum or not. Kim et al. [9] found an elegant way to reduce the corresponding computational effort. They have shown for the two dimensional case, that a local maximum or minimum is only possible at one of the four corners of a volume, respectively. Thus only two corner value have to be checked, $q_{\text {imax,jmax }}^{M L P}$ to be a local maximum and $q_{i m i n, j m i n}^{M L P}$ to be a minimum. Depending on $\Delta q_{x}^{m m}$ and $\Delta q_{y}^{m m}$ the locations of the vertex points (imax, jmax) and (imin,jmin) to be checked are

\begin{tabular}{|c|c|c|c|c|c|}
\hline & $i \max$ & imin & & $j \max$ & jmin \\
\hline$\Delta q_{x}^{m m}>0$ & $i+\frac{1}{2}$ & $i-\frac{1}{2}$ & $\Delta q_{y}^{m m}>0$ & $j+\frac{1}{2}$ & $j-\frac{1}{2}$ \\
\hline$\Delta q_{x}^{m m}<0$ & $i-\frac{1}{2}$ & $i+\frac{1}{2}$ & $\Delta q_{y}^{m m}<0$ & $j-\frac{1}{2}$ & $j+\frac{1}{2}$ \\
\hline
\end{tabular}

For $\Delta q^{m m}=0$ in one or both coordinate directions, a local extremum at one of the corners of the volume becomes impossible [1] (the cell center $i, j$ already is an extremum). In this case $\alpha_{x}=\alpha_{y}=2$ can be used and there is no limitation due to MLP in the remaining coordinate direction. Due to the fact that only two vertex points have to be checked with one condition each, Eq. (30) is replaced by

$$
Q_{i m i n, j \min }^{\min } \leq q_{i m i n, j m i n}^{M L P}, \quad \quad q_{i m a x, j m a x}^{M L P} \leq Q_{\text {imax }, \text { maxax }}^{\max }
$$

Inserting Eq. (29) and taking positive gradients to check for a maximum and negative ones to check for a minimum

$$
\begin{aligned}
\alpha_{x}\left|\Delta q_{x}^{m m}\right|+\alpha_{y}\left|\Delta q_{y}^{m m}\right| & \leq 2\left(Q_{i m a x, j m a x}^{\max }-q_{i, j}\right), \\
-\alpha_{x}\left|\Delta q_{x}^{m m}\right|-\alpha_{y}\left|\Delta q_{y}^{m m}\right| & \geq 2\left(Q_{i m i n, j \min }^{\min }-q_{i, j}\right)
\end{aligned}
$$

are obtained. Finally both criteria are combined to

$$
\begin{aligned}
\left|\Delta q^{M L P}\right| & =\alpha_{x}\left|\Delta q_{x}^{m m}\right|+\alpha_{y}\left|\Delta q_{y}^{m m}\right| \\
& \leq 2 \min \left(Q_{\text {imax }, j \max }^{\max }-q_{i, j}, q_{i, j}-Q_{i m i n, j m i n}^{\min }\right)=\Delta q^{c} .(35)
\end{aligned}
$$


This criterion has to be fulfilled to satisfy the MLP condition. The newly defined term $\Delta q^{c}$ is a limiting value for the interface state reconstruction. The added reconstructed interface differences from both coordinate directions (linear assumption) must be below this value as to avoid a local extremum. It is easy to show, that for $\alpha_{x}=\alpha_{y}=1$ condition (35) is always satisfied, independently from the values of the diagonal volumes. This means that in $2 \mathrm{D}$ the minmod limiter always satisfies the MLP condition.

Proof: If there is no local extremum $\left(r_{i} \geq 0\right)$ it follows from Eqs. (28) and (12) that

$$
\begin{aligned}
\left|\Delta q_{x}^{m m}\right| & =\max \left[0, \min \left(1, r_{i}\right)\right]\left|\Delta q_{i-1 / 2, j}\right| \\
& =\min \left(\left|\Delta q_{i-1 / 2, j}\right|,\left|\Delta q_{i+1 / 2, j}\right|\right)
\end{aligned}
$$

and with this result and the index definition

\begin{tabular}{lcc} 
& Imax & Imin \\
\hline$\Delta q_{x}^{m m}>0$ & $i+1$ & $i-1$ \\
$\Delta q_{x}^{m m}<0$ & $i-1$ & $i+1$ \\
\hline
\end{tabular}

\begin{tabular}{llll} 
& Jmax & Jmin \\
\hline$\Delta q_{y}^{m m}>0$ & $j+1$ & $j-1$ \\
$\Delta q_{y}^{m m}<0$ & $j-1$ & $j+1$
\end{tabular}

it follows from Eq. (26) that

$$
\begin{aligned}
2\left(Q_{i \max , j \max }^{\max }-q_{i, j}\right) & =2\left[\max \left(q_{i, j}, q_{\text {Imax }, j}, q_{i, J \max }, q_{\text {Imax }, J \max }\right)-q_{i, j}\right] \\
& \geq 2 \max \left[0, q_{I \max , j}-q_{i, j}, q_{i, J \max }-q_{i, j}\right] \\
& \geq 2 \max \left[\min \left(\left|\Delta q_{i-1 / 2, j}\right|,\left|\Delta q_{i+1 / 2, j}\right|\right), \min \left(\left|\Delta q_{i, j-1 / 2}\right|,\left|\Delta q_{i, j+1 / 2}\right|\right)\right](38)
\end{aligned}
$$

By inserting the last result, Eq. (36), and $\alpha_{x}=\alpha_{y}=1$ in Eq. (33)

$$
\begin{aligned}
& \min \left(\left|\Delta q_{i-1 / 2, j}\right|,\left|\Delta q_{i+1 / 2, j}\right|\right)+\min \left(\left|\Delta q_{i, j-1 / 2}\right|,\left|\Delta q_{i, j+1 / 2}\right|\right) \\
\leq & 2 \max \left[\min \left(\left|\Delta q_{i-1 / 2, j}\right|,\left|\Delta q_{i+1 / 2, j}\right|\right), \min \left(\left|\Delta q_{i, j-1 / 2}\right|,\left|\Delta q_{i, j+1 / 2}\right|\right)(39)\right.
\end{aligned}
$$

is obtained. This condition corresponds to $|a|+|b| \leq 2 \max (|a|,|b|)$, which is always satisfied. The proof for the minimum is identical and thus Eq. (35) is proved for $\alpha_{x}=\alpha_{y}=1$. 
In two-dimensional flow there is another property of MLP, which simplifies the procedure: If there is a local extremum $\left(r_{i}<0\right)$ in one direction of the flow, $\alpha=2$ (the upper MLP limit) may be used in the other direction.

Proof: With $r_{x}<0$ there is a maximum or minimum in the $x$-direction and from the minmod limiter defined by Eq. (19) $\Delta q_{x}^{m m}=0$ is obtained. With $r_{y}>0$ in the other direction (otherwise the proof is trivial) it follows from Eq. (36) that

$$
\left|\Delta q_{y}^{m m}\right|=\min \left(\left|\Delta q_{i, j-1 / 2}\right|,\left|\Delta q_{i, j+1 / 2}\right|\right)
$$

and from Eq. (38)

$$
2\left(Q_{i m a x, j \max }^{\max }-q_{i, j}\right) \geq 2 \min \left(\left|\Delta q_{i, j-1 / 2}\right|,\left|\Delta q_{i, j+1 / 2}\right|\right) .
$$

Inserting both results into Eq. (35) achieves

$$
\alpha_{y} \min \left(\left|\Delta q_{i, j-1 / 2}\right|,\left|\Delta q_{i, j+1 / 2}\right|\right) \leq 2 \min \left(\left|\Delta q_{i, j-1 / 2}\right|,\left|\Delta q_{i, j+1 / 2}\right|\right)
$$

which is valid for $\alpha_{y}=2$.

\subsection{A modified MLP version - MLP ${ }^{l d}$}

The previous section has shown, that by an appropriate choice of the MLP parameters $\alpha_{x}$ and $\alpha_{y}$, local extrema at the corner points of a volume can be avoided. The question is now, how $\alpha_{x}$ and $\alpha_{y}$ are determined. With Eq. (35) there is only one condition for two unknown parameters which not necessarily have to have the same value. In Fig. 3 the situation is shown for $r_{x}, r_{y} \geq 0$. With $\alpha_{x}, \alpha_{y} \in[1,2]$ it follows from Eq. (28) that the possible absolute changes in $x$ - and $y$-direction, allowed by MLP, are between one and two times the changes, caused by the minmod limiter. This corresponds to the thick drawn rectangle plotted in Fig. 3. If the $\leq$ sign in Eq. (35) is replaced by the equality sign, the upper limit for $\alpha_{x}, \alpha_{y}$ is taken to avoid excessive numerical dissipation and

$$
\left|\Delta q^{M L P}\right|=\alpha_{x}\left|\Delta q_{x}^{m m}\right|+\alpha_{y}\left|\Delta q_{y}^{m m}\right|=\left|\Delta q_{x}^{M L P}\right|+\left|\Delta q_{y}^{M L P}\right|=\Delta q^{c}
$$




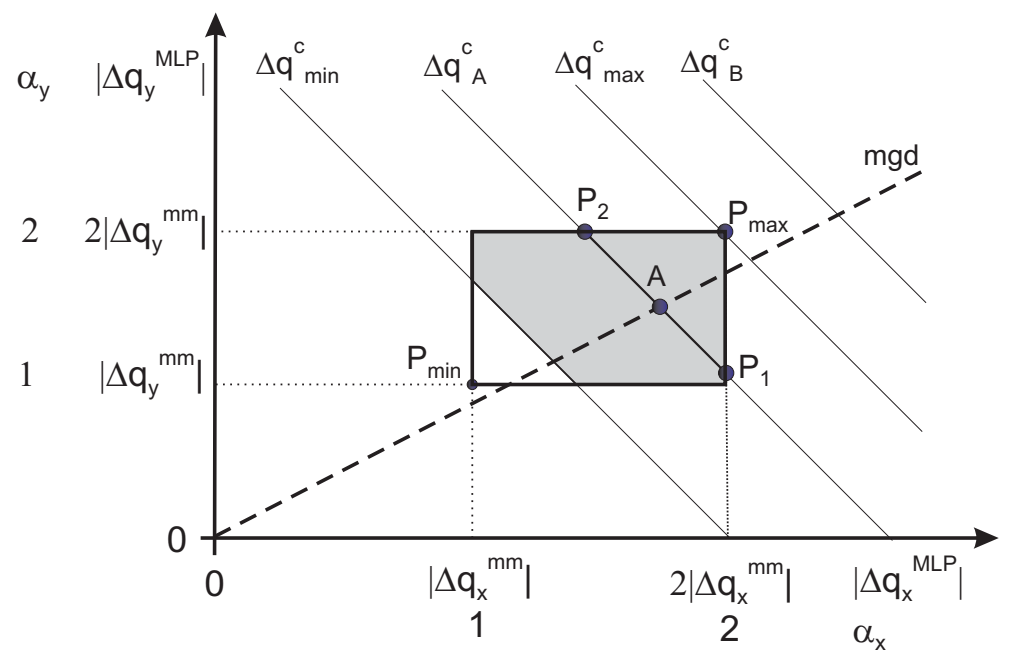

Figure 3: Possible two-dimensional MLP region (shaded section) and limiting lines $\Delta q^{c}$ due to the MLP criteria. The dashed line is the mean gradient direction $(m g d)$ and point $\mathbf{A}$ is the solutions in case of $\Delta q_{\mathbf{A}}^{c}$.

is obtained. According to this equation, a given point $\left|\Delta q_{x}^{M L P}\right|,\left|\Delta q_{y}^{M L P}\right|$ in the diagram of Fig. 3 directly corresponds to a set of $\alpha_{x}=\left|\Delta q_{x}^{M L P}\right| /\left|\Delta q_{x}^{m m}\right|$ and $\alpha_{y}=\left|\Delta q_{y}^{M L P}\right| /\left|\Delta q_{y}^{m m}\right|$ values. Using Eqs. (35), (31) and (26) the limiting factor $\Delta q^{c}$ can be calculated easily for any volume. For a given $\Delta q^{c}$ the linear relationship (43) defines a line with slope minus one in $\left|\Delta q_{x}^{M L P}\right|-\left|\Delta q_{y}^{M L P}\right|$-space. In Fig. 3 examples of such limiting lines are plotted. As proved before, $\Delta q^{c} \geq$ $\left|\Delta q_{x}^{m m}\right|+\left|\Delta q_{y}^{m m}\right|$. However,

$$
\Delta q^{c} \geq \Delta q_{\min }^{c}=2 \max \left(\left|\Delta q_{x}^{m m}\right|,\left|\Delta q_{y}^{m m}\right|\right)
$$

is a more important limit which generally defines the lowest possible value for $\Delta q^{c}$. The corresponding proof has already been given by Eq. (38). It follows that a simultaneous MLP limitation to $\alpha_{x}=\alpha_{y}=1$ (minmod limiter) in both coordinate directions is possible for $\left|\Delta q_{x}^{m m}\right|=\left|\Delta q_{y}^{m m}\right|$ only. For $\left|\Delta q_{x}^{m m}\right| \neq$ $\left|\Delta q_{y}^{m m}\right|$ the line $\Delta q_{\min }^{c}$ narrows the MLP region $\left(\alpha_{x}, \alpha_{y} \in[1,2]\right)$ to the dark shaded area plotted in Fig. 3, which later on is called realizable or possible MLP region. For $\left|\Delta q_{x}^{m m}\right|=\left|\Delta q_{y}^{m m}\right|=\left|\Delta q^{m m}\right|$ a square MLP region is obtained. In 
this case the lower limiting line $\Delta q^{c}=\Delta q_{\text {min }}^{c}$ collapses into a single possible solution in the MLP region, given by $\mathbf{P}_{\min }=\mathbf{P}\left(\left|\Delta q^{m m}\right|,\left|\Delta q^{m m}\right|\right)$. If, on the other hand, $\Delta q^{c} \geq 2\left(\left|\Delta q_{x}^{m m}\right|+\left|\Delta q_{y}^{m m}\right|\right)=\Delta q_{\max }^{c}$ (see lines $\Delta q_{\max }^{c}$ and $\left.\Delta q_{B}^{c}\right)$, the upper MLP limit is reached or exceeded and with $\mathbf{P}_{\max }$ and $\alpha_{x}=\alpha_{y}=2$ there again is a single solution only. The question is what happens, if $\Delta q^{c}$ is located between the lower limit $\Delta q_{\min }^{c}$ and the upper limit $\Delta q_{\max }^{c}$ ? In this case any point on the line of constant $\Delta q^{c}$ crossing the shaded area fulfills the MLP condition. In case of $\Delta q_{\mathbf{A}}^{c}$ (see Fig. 3), any point between $\mathbf{P}_{1}$ and $\mathbf{P}_{2}$ could be chosen. Obviously this choice has a strong influence on the gradient of the reconstructed interface values. Hence the MLP condition can be satisfied $\left(\left|\Delta q^{M L P}\right|=\Delta q^{c}\right)$ and at the same time a certain gradient $\left|\Delta q_{y}^{M L P}\right| /\left|\Delta q_{x}^{M L P}\right|$ of the interface values can be created within some given limits. The most simple choice is $\alpha_{x}=\alpha_{y}$. In this case the changes in both coordinate directions are reduced by MLP (if necessary) with the same factor. However, in practical tests this measure caused a stall of convergence. Thus the present choice is to create a gradient for the interface values, which corresponds as far as possible to the mean gradient of the flow variable. The absolute mean gradient of a variable $q$

$$
\bar{\gamma}_{y x}=\left|\frac{\Delta \bar{q}_{y}}{\Delta \bar{q}_{x}}\right|=\left|\frac{q_{i, j+1}-q_{i, j-1}}{q_{i+1, j}-q_{i-1, j}}\right|
$$

is calculated by second order central differences [1]. Even for the fourth and fifth order schemes this spatially second order approximation was found to be sufficient and a fourth order central discretization achieved no improvements. The line of absolute mean gradient direction (mgd) $\left|\Delta q_{y}^{M L P}\right|=\bar{\gamma}_{y x}\left|\Delta q_{x}^{M L P}\right|$ is used as a second condition to define the ratio between $\alpha_{x}$ and $\alpha_{y}$. In Fig. 3 a possible line for the mean gradient direction (the dashed line indicated with mgd) is plotted. The point of intersection $\mathbf{A}=\mathbf{A}\left(A_{x}, A_{y}\right)$ between the line of absolute mean gradient direction with the limiting line $\left|\Delta q^{M L P}\right|=\Delta q^{c}=$ const. can be easily calculated by

$$
A_{x}=\frac{\Delta q^{c}}{1+\bar{\gamma}_{y x}}, \quad A_{y}=\bar{\gamma}_{y x} A_{x} .
$$

This point (A in Fig. 3 for the limiting line $\Delta q_{\mathbf{A}}^{c}$ ) fulfills the following conditions: 

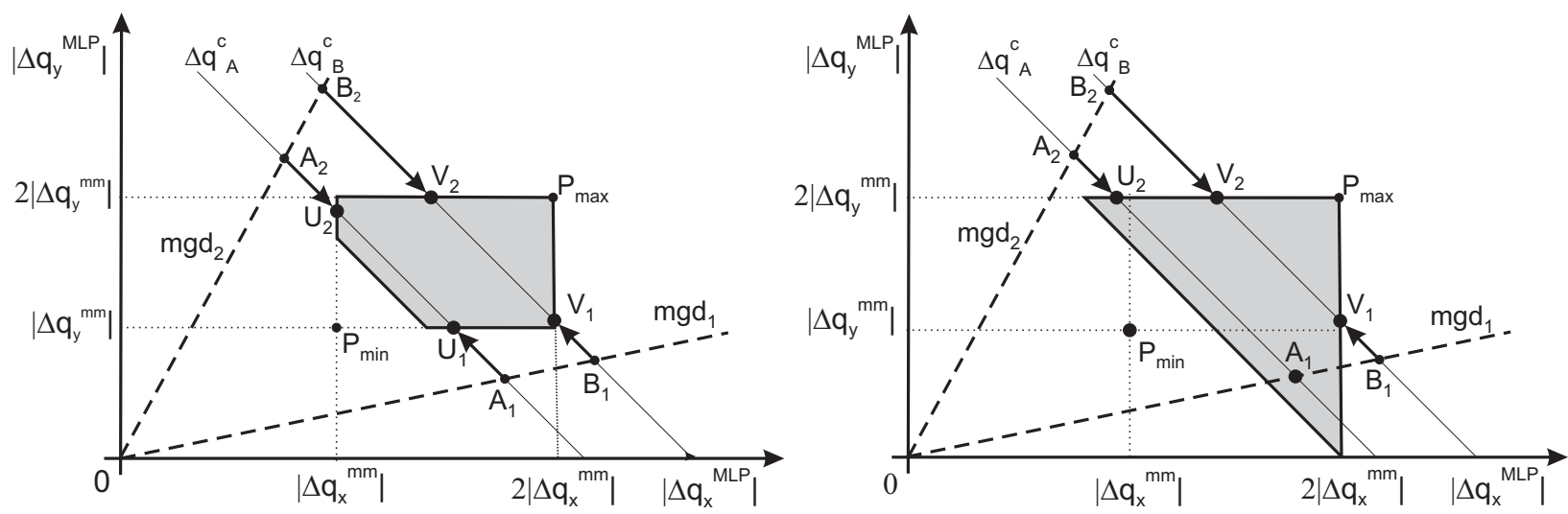

Figure 4: Realizable MLP regions (dark shaded sections) in two-dimensional flow in case of $\alpha_{x}, \alpha_{y} \in[1,2]$ (left side) and $\alpha_{x}, \alpha_{y} \in[0,2]$ (right side). Lines of constant $\Delta q^{c}$ are possible limits due to the MLP condition and the dashed lines $\left(m g d_{1}\right.$ or $\left.m g d_{2}\right)$ indicate possible mean gradient directions. If the points of intersection $\mathbf{A}_{i}$ or $\mathbf{B}_{i}$ are outside the MLP region, they are shifted back to the outer MLP boundary (to the points $\mathbf{U}_{i}$ or $\mathbf{V}_{i}$ ) while keeping $\left|\Delta q^{M L P}\right|=\Delta q_{\mathbf{A}, \mathbf{B}}^{c}$ constant.

1. The MLP criteria is satisfied and the interface value reconstruction does not cause a local extremum at any of the corners of the volume.

2. Maximum values for $\alpha_{x}$ and $\alpha_{y}$ are obtained by using $\left|\Delta q^{M L P}\right|=\Delta q^{c}$.

3. The reconstructed interface values have a gradient which corresponds to the mean gradient direction.

The compliance of all conditions can be achieved only, if the point of intersection is in the possible MLP region, given by the shaded area in Fig. 3. As long as the flowfield is relatively smooth this usually is the case. Close to a discontinuity however, large deviations may occur between the gradients caused by mean and interface values. It even becomes possible, that the line of the mean gradient direction does not cross the MLP region at all. Corresponding situations are plotted on the left side of Fig. 4. Both lines of mean gradient directions ( $m g d_{1}$ and $m g d_{2}$ ) do not cross the MLP region and one of the last two above given requirements has to be skipped. In our newly proposed version, which is called $\mathrm{MLP}^{l d}$ (low diffusion), the highest possible value for $\left|\Delta q^{M L P}\right|$ (defined by Eq. 
(43)) is required $\left(\left|\Delta q^{M L P}\right|=\Delta q^{c}\right)$. This is an important feature because in this way the highest possible values for $\alpha_{x}$ and $\alpha_{y}$ are obtained, independently from the mean gradient direction. Thus the third condition has to be skipped. As to fulfill the first two conditions, the point of intersection of the line $\left|\Delta q^{M L P}\right|=$ $\Delta q^{c}=$ const. with the outer MLP boundary is sought. In case of the limiting condition $\Delta q_{\mathbf{A}}^{c}$ and the mean gradient direction $m g d_{1}$ (see Fig. 4 left side) point $\mathbf{A}_{1}$ is shifted to $\mathbf{U}_{1}$ which is the final solution. While in this way the first two conditions given above are fulfilled, the direction of the limited interface values (see line $\mathbf{O}-\mathbf{U}_{1}$ in Fig. 4, left side) deviates from the mean gradient direction $m g d_{1}$.

Even if the third condition is skipped, a stabilizing effect was observed if the gradient of the interface values is close to the mean gradient direction. A better agreement between both directions can be achieved, if the requirement $\alpha_{x}, \alpha_{y} \in$ $[1,2]$ is released to $\alpha_{x}, \alpha_{y} \in[0,2]$. This causes more numerical dissipation in the vicinity of discontinuities. However, practical investigations have shown, that this measure improves the level of convergence significantly, while differences in the variable profiles where hardly visible. Thus, if not stated otherwise, in this paper $\alpha_{x}, \alpha_{y} \in[0,2]$ is used for all simulations. Mathematically the shift (if necessary) back to the outer MLP boundary is easy to perform. First the coordinates of $\mathbf{A}=\mathbf{A}\left(A_{x}, A_{y}\right)$ (or $\mathbf{B}$ ) are calculated according to Eq. (46) from which the coordinates of $\mathbf{U}=\mathbf{U}\left(U_{x}, U_{y}\right)$ (or $\mathbf{V}$ ) are obtained by

$$
\begin{aligned}
\left|\Delta q_{x y}\right| & =-\max \left(A_{x}-2\left|\Delta q_{x}^{m m}\right|, 0\right)+\max \left(A_{y}-2\left|\Delta q_{y}^{m m}\right|, 0\right) \\
U_{x} & =A_{x}+\left|\Delta q_{x y}\right| \\
U_{y} & =A_{y}-\left|\Delta q_{x y}\right| .
\end{aligned}
$$

If $\mathbf{A}$ already is inside the realizable MLP boundary no shift is performed by this measure.

The effect of such a shift on the gradient of the interface values is illustrated on the right side of Fig. 4. The dark shaded area is the possible MLP region in case of $\alpha_{x}, \alpha_{y} \in[0,2]$. For the points $\mathbf{B}_{1}$ and $\mathbf{B}_{2}$ the situation remains the same as in case of $\alpha_{x}, \alpha_{y} \in[1,2]$ and points $\mathbf{V}_{1}$ and $\mathbf{V}_{2}$ deliver the final $\alpha_{x}$ and $\alpha_{y}$ 
values. For the points $\mathbf{A}_{1}$ and $\mathbf{A}_{2}$ however, the situation differs. While on the left side of Fig. 4 point $\mathbf{A}_{1}$ is located outside the realizable MLP region, it is inside in case of the extended MLP region (right figure). In the latter case $\mathbf{A}_{1}$ directly delivers final $\alpha_{x}$ and $\alpha_{y}$ values. The gradients of the mean and interface values are identical at the price, that $\alpha_{y}$ takes a value below one. In case of $\mathbf{A}_{2}$ a shift to $\mathbf{U}_{2}$ is performed. The corresponding $\alpha_{x}$ value of $\mathbf{U}_{2}$ is below one, but again, the difference to the mean gradient direction is smaller than in case of $\alpha_{x}, \alpha_{y} \in[1,2]$. Due to the lower limiting line $\Delta q_{\min }^{c}$ the differences in the possible MLP areas between both approaches (left and right side of Fig. 4) are much smaller, than it could expected by a change from $\alpha_{x}, \alpha_{y} \in[1,2]$ to $\alpha_{x}, \alpha_{y} \in[0,2]$. The MLP condition $\left|\Delta q^{M L P}\right|=\Delta q^{c}$ is always satisfied independently from the definition of the $\alpha_{i}$-range. Because $\Delta q^{c}$ is kept constant in $\mathrm{MLP}^{l d}$, a reduction of $\alpha_{i}$ to values below one in one coordinate direction (by the shift to the outer MLP boundary) causes an identical increase of $\alpha_{j}, j \neq i$ in the remaining direction. This is in contrast to the standard MLP version, where the $\alpha_{j}$ values are not increased. Moreover, it has to be taken into account, that these limitations are performed only, if there is local extremum at a vertex point of the volume.

\section{$\operatorname{MLP}^{l d}$ procedure for the calculation of $\alpha_{x}$ and $\alpha_{y}$ in two-dimensional \\ flows}

Calculate $\left|\Delta q_{x}^{m m}\right|$ and $\left|\Delta q_{y}^{m m}\right|$ from Eq. (28), $\Delta q^{c}$ from the right part of Eq. (35), $\left|\Delta \bar{q}_{x}\right|,\left|\Delta \bar{q}_{y}\right|$ and $\bar{\gamma}_{y x}$ from Eq. (45), and set $\alpha_{x}=\alpha_{y}=2$.

$$
\alpha_{x}, \alpha_{y} \in[0,2]\left\{\begin{aligned}
& \text { if }\left(2\left(\left|\Delta q_{x}^{m m}\right|+\left|\Delta q_{y}^{m m}\right|\right)>\Delta q^{c}\right) \text { then } \\
&\left|\Delta q_{x}\right|=\Delta q^{c} /\left(1+\bar{\gamma}_{y x}\right), \quad\left|\Delta q_{y}\right|=\left|\Delta q_{x}\right| \bar{\gamma}_{y x}, \\
&\left|\Delta q_{x y}\right|=-\max \left(\left|\Delta q_{x}\right|-2\left|\Delta q_{x}^{m m}\right|, 0\right)+\max \left(\left|\Delta q_{y}\right|-2\left|\Delta q_{y}^{m m}\right|, 0\right), \\
&\left|\Delta q_{x}\right|=\left|\Delta q_{x}\right|+\left|\Delta q_{x y}\right|, \quad\left|\Delta q_{y}\right|=\left|\Delta q_{y}\right|-\left|\Delta q_{x y}\right|, \\
& \alpha_{x}=\left|\Delta q_{x}\right| /\left|\Delta q_{x}^{m m}\right|, \quad \alpha_{y}=\left|\Delta q_{y}\right| /\left|\Delta q_{y}^{m m}\right| \\
& \text { end if }
\end{aligned}\right.
$$


As mentioned before, in 2D the MLP condition can already be satisfied with $\alpha_{x}, \alpha_{y} \in[1,2]$. The corresponding procedure requires additional checks at the lower MLP boundaries

$$
\alpha_{x}, \alpha_{y} \in[1,2]\left\{\begin{aligned}
\text { if }\left(2 \left(\left|\Delta q_{x}^{m m}\right|\right.\right. & \left.\left.+\left|\Delta q_{y}^{m m}\right|\right)>\Delta q^{c}\right) \text { then } \\
\bar{\gamma}_{y x} & =\min \left[\max \left(\bar{\gamma}_{y x}, 0.5\left|\Delta q_{y}^{m m}\right| / \Delta q_{x}^{m m} \mid\right), 2\left|\Delta q_{y}^{m m}\right| /\left|\Delta q_{x}^{m m}\right|\right], \\
\left|\Delta q_{x}\right| & =\Delta q^{c} /\left(1+\bar{\gamma}_{y x}\right),\left|\Delta q_{y}\right|=\left|\Delta q_{x}\right| \bar{\gamma}_{y x}, \\
\left|\Delta q_{x y}\right| & =\max \left(\left|\Delta q_{x}^{m m}\right|-\left|\Delta q_{x}\right|, 0\right)-\max \left(\left|\Delta q_{x}\right|-2\left|\Delta q_{x}^{m m}\right|, 0\right) \\
& -\max \left(\left|\Delta q_{y}^{m m}\right|-\left|\Delta q_{y}\right|, 0\right)+\max \left(\left|\Delta q_{y}\right|-2\left|\Delta q_{y}^{m m}\right|, 0\right), \\
\left|\Delta q_{x}\right| & =\left|\Delta q_{x}\right|+\left|\Delta q_{x y}\right|,\left|\Delta q_{y}\right|=\left|\Delta q_{y}\right|-\left|\Delta q_{x y}\right|, \\
\alpha_{x} & =\left|\Delta q_{x}\right| /\left|\Delta q_{x}^{m m}\right|, \quad \alpha_{y}=\left|\Delta q_{y}\right| /\left|\Delta q_{y}^{m m}\right|
\end{aligned}\right.
$$

In practice however, the more simple approach from Eq. (48) was found to be sufficient and thus is recommended. The calculated $\alpha_{x}$ and $\alpha_{y}$ values may be used in Eq. (23) in combination with any higher order approach for $\beta$.

\subsection{The MLP version of Kim et al. [1]}

The derivation of the newly proposed version $\mathrm{MLP}^{l d}$ basically follows the work of Kim et al. [1]. Different however, is the adaption of the interface values to the direction of the mean gradient outside the defined MLP region. Kim et al. start from Eq. (43) using a single parameter $\alpha$

$$
\alpha\left|\Delta q_{x}^{m m}\right|+\alpha\left|\Delta q_{y}^{m m}\right|=\Delta q^{c}
$$

By introducing $r_{y x}=\left|\Delta q_{y}^{m m}\right| /\left|\Delta q_{x}^{m m}\right|$ and $r_{x y}=1 / r_{y x}$ two identical equations

$$
\begin{aligned}
& \alpha\left(1+r_{y x}\right)\left|\Delta q_{x}^{m m}\right|=\Delta q^{c}, \quad x-\text { direction }, \\
& \alpha\left(r_{x y}+1\right)\left|\Delta q_{y}^{m m}\right|=\Delta q^{c}, \quad y-\text { direction }
\end{aligned}
$$

are formulated. Later on $r_{y x}$ is approximated by $r_{y x} \approx \bar{\gamma}_{y x}$ using $\bar{\gamma}_{y x}$ from Eq. (45) and $r_{x y}$ is approximated by $r_{x y} \approx \bar{\gamma}_{x y}=1 / \bar{\gamma}_{y x}$. Thus different values in the $x$ - and $y$-direction

$$
\alpha_{x}=\Delta q^{c} /\left[\left(1+\bar{\gamma}_{y x}\right)\left|\Delta q_{x}^{m m}\right|\right],
$$




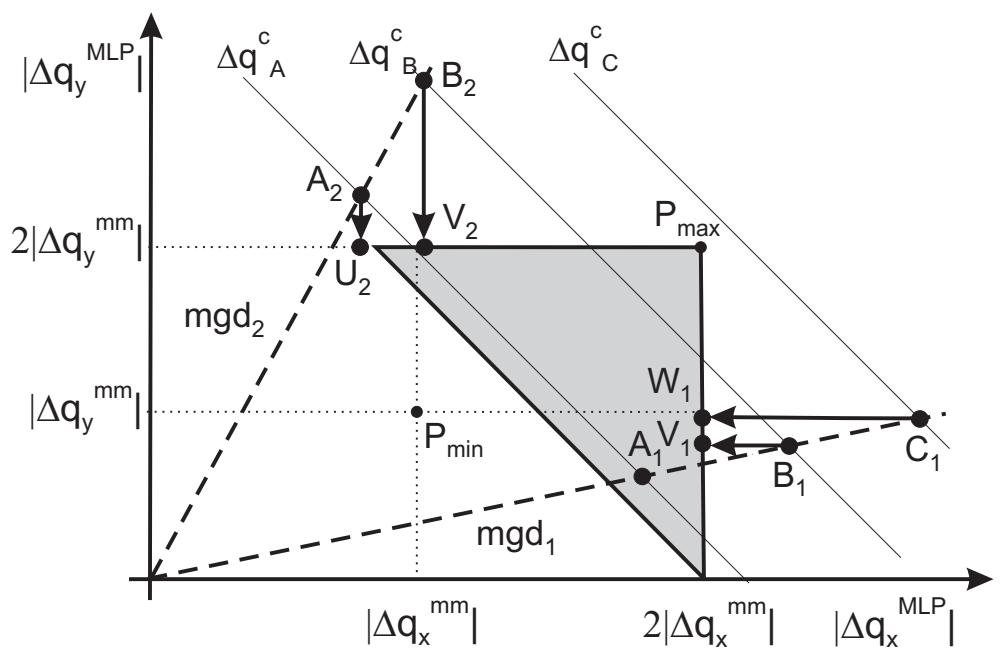

Figure 5: Possible MLP region (dark shaded area) in two-dimensional flow for the MLP version of Kim et al. [1] and the assumption $\alpha_{x}, \alpha_{y} \in[0,2]$. Shown are situations where the intersections between limiting lines $\Delta q^{c}$ and mean gradient directions $(m g d)$ are inside $\left(\mathbf{A}_{1}\right)$ or outside (the remaining points) the possible MLP region. Final solutions for outside points are obtained by horizontal and vertical shifts back to the outer MLP boundary.

$$
\alpha_{y}=\Delta q^{c} /\left[\left(\bar{\gamma}_{x y}+1\right)\left|\Delta q_{y}^{m m}\right|\right]
$$

are obtained. As before in case of $\mathrm{MLP}^{l d}$, the ratio between $\alpha_{x}$ and $\alpha_{y}$ and therefore the gradient of the reconstructed interface values is defined by the mean gradient direction $\bar{\gamma}_{y x}$.

Proof: Based on different values for $\alpha_{x}$ and $\alpha_{y}$, Eq. (43) is reformulated to

$$
\begin{aligned}
& \alpha_{x}=\Delta q^{c} /\left(\left|\Delta q_{x}^{m m}\right|+\left|\Delta q_{y}^{m m}\right| \alpha_{y} / \alpha_{x}\right), \\
& \alpha_{y}=\Delta q^{c} /\left(\left|\Delta q_{x}^{m m}\right| \alpha_{x} / \alpha_{y}+\left|\Delta q_{y}^{m m}\right|\right) .
\end{aligned}
$$

Equations (52) are identical to Eqs. (53) for

$$
\frac{\alpha_{y}}{\alpha_{x}} \frac{\left|\Delta q_{y}^{m m}\right|}{\left|\Delta q_{x}^{m m}\right|}=\bar{\gamma}_{y x} \text {. }
$$

Hence the ratio between the MLP limited interface values $\alpha_{y}\left|\Delta q_{y}^{m m}\right|$ and $\alpha_{x}\left|\Delta q_{x}^{m m}\right|$ corresponds to the direction of the mean gradient.

The proof is valid only, if no additional limitations are performed. In practice, $\alpha_{x}$ and $\alpha_{y}$ at least have to be limited to $\alpha_{x}, \alpha_{y} \leq 2$. In addition $\alpha_{x}$ and $\alpha_{y}$ 
can be limited to $\alpha_{x}, \alpha_{y} \geq 1$. However, the last limitation causes a discontinuous behaviour of $\alpha_{x}$ and $\alpha_{y}$. The realizable MLP region without any lower limitation is plotted in Fig. 5 by the dark shaded area. The newly proposed versions $\mathrm{MLP}^{l d}$ and the approach of Kim et al. [1] are identical, as long as the point of intersection between the mean gradient and the limiting line $\Delta q^{c}=$ const. is located in the realizable MLP region. If this is not the case, significant differences are possible. In the approach of Kim et al. [1] points located outside are shifted horizontally or vertically back to the boundary of the MLP region. This has the disadvantage, that $\Delta q^{c}$ is reduced instead of kept constant. Thus, $\alpha_{x}$ and $\alpha_{y}$ take values, which are smaller than required by the MLP condition. Therefore close to discontinuities, this version is expected to be more dissipative than necessary. Two examples are shown in Fig. 5 (using $\alpha_{x}, \alpha_{y} \in[0,2]$ ) where the points $\mathbf{B}_{1}$ and $\mathbf{B}_{2}$ are shifted to $\mathbf{V}_{1}$ and $\mathbf{V}_{2}$, respectively. In case of $m g d_{1}$ and $\Delta q_{\mathbf{A}}^{c}$ point $\mathbf{A}_{1}$ is the point of intersection which already is in the MLP region. In the MLP version of Kim et al. [1], $\alpha$ values below two are possible even in cases, where actually no limitation is needed due to the MLP constraint. In Fig. 5 such a situation is shown for $m g d_{1}$ and $\Delta q_{\mathbf{C}}^{c}$. The point of intersection $\mathbf{C}_{1}$ is shifted to the border of the MLP region (point $\mathbf{W}_{1}$ ) with final values of $\alpha_{x}=2$ and $\alpha_{y}<1$. However, $\Delta q_{\mathbf{C}}^{c}$ is above the MLP limit and no limitation is needed. This problem arises because $\Delta q_{\mathbf{C}}^{c}$ is not kept constant. As long as $\alpha_{x}>2$ and $\alpha_{y}<2$ or reverse, limitation takes place independently from $\Delta q^{c}$. Moreover it is possible, that the final $\alpha_{x}$ and $\alpha_{y}$ values are outside the shaded MLP region, as shown in another example in Fig. 5. Point $\mathbf{A}_{2}$ is shifted to the boundary point $\mathbf{U}_{2}$ and $\Delta q_{\mathbf{U}_{2}}^{c}<\Delta q_{\min }^{c}$. If the mean gradient direction approaches the $x$ - or the $y$-axis, these problems increase. Of course some of these effects may be corrected by additional limitations. On the other hand, the MLP scheme of Kim et al. [1] is more simple to program. The savings in CPU time however, are negligible. 


\section{Three-dimensional MLP}

In the three-dimensional case the basics of MLP remain the same. Again, local extrema at the corners of a volume are avoided by choosing appropriate $\alpha$ values. Now eight corners have to be checked for any volume $i, j, k$ and with $\kappa_{x}, \kappa_{y}, \kappa_{z}= \pm 1$ it follows from Eqs. (25) and (26)

$$
Q_{i+\kappa_{x} / 2, j+\kappa_{y} / 2, k+\kappa_{z} / 2}^{\min } \leq q_{i+\kappa_{x} / 2, j+\kappa_{y} / 2, k+\kappa_{z} / 2} \leq Q_{i+\kappa_{x} / 2, j+\kappa_{y} / 2, k+\kappa_{z} / 2}^{\max }
$$

where

$$
\begin{aligned}
& Q_{i+\kappa_{x} / 2, j+\kappa_{y} / 2, k+\kappa_{z} / 2}^{\min }= \min \left(q_{i, j, k}, q_{i+\kappa_{x}, j, k}, q_{i, j+\kappa_{y}, k}, q_{i+\kappa_{x}, j+\kappa_{y}, k}, q_{i, j, k+\kappa_{z}}, q_{i+\kappa_{x}, j, k+\kappa_{z}},\right. \\
&\left.q_{i, j+\kappa_{y}, k+\kappa_{z}}, q_{i+\kappa_{x}, j+\kappa_{y}, k+\kappa_{z}}\right), \\
& Q_{i+\kappa_{x} / 2, j+\kappa_{y} / 2, k+\kappa_{z} / 2}^{\max }=\max \left(q_{i, j, k}, q_{i+\kappa_{x}, j, k}, q_{i, j+\kappa_{y}, k}, q_{i+\kappa_{x}, j+\kappa_{y}, k}, q_{i, j, k+\kappa_{z}}, q_{i+\kappa_{x}, j, k+\kappa_{z}},\right. \\
&\left.q_{i, j+\kappa_{y}, k+\kappa_{z}}, q_{i+\kappa_{x}, j+\kappa_{y}, k+\kappa_{z}}\right) .
\end{aligned}
$$

The upper and lower limits $Q^{\max }$ and $Q^{\min }$ are obtained for any corner of a volume by taking the minimum or maximum out of the eight surrounding cell center values. To check for a local extremum, the interface values are linearly combined by

$q_{i+\kappa_{x} / 2, j+\kappa_{y} / 2, k+\kappa_{z} / 2}^{M L P}=q_{i, j, k}+0.5 \kappa_{x} \alpha_{x} \Delta q_{x}^{m m}+0.5 \kappa_{y} \alpha_{y} \Delta q_{y}^{m m}+0.5 \kappa_{z} \alpha_{z} \Delta q_{z}^{m m}$,

to a corner MLP value. As in the two dimensional case (see Eq. (28)) $\Delta q_{x}^{m m}$, $\Delta q_{y}^{m m}, \Delta q_{z}^{m m}$ are the differences caused by the minmod limiter in the corresponding coordinate direction, respectively. The MLP corner values have to fulfill the following condition

$$
Q_{i+\kappa_{x} / 2, j+\kappa_{y} / 2, k+\kappa_{z} / 2}^{\min } \leq q_{i+\kappa_{x} / 2, j+\kappa_{y} / 2, k+\kappa_{z} / 2}^{M L P} \leq Q_{i+\kappa_{x} / 2, j+\kappa_{y} / 2, k+\kappa_{z} / 2}^{\max }
$$

from which $\alpha_{x}, \alpha_{y}$, and $\alpha_{z}$ are derived. Again not all corners of a volume have to be checked for a local extremum. However, in contrast to the two-dimensional case and what is stated by Kim et al. [1], in 3D two corner points may cause 
a local maximum and two a local minimum. The first corner to be checked for a maximum is $\mathbf{C}_{1}^{\max }$ located at imax, jmax, $k \max$ and for a minimum $\mathbf{C}_{1}^{\min }$ located at imin, jmin, kmin. In the same way as in 2D the corner indices

\begin{tabular}{|c|c|c|c|c|c|c|c|c|}
\hline & $i \max$ & imin & & $j \max$ & jmin & & $k \max$ & $k m i n$ \\
\hline$\Delta q_{x}^{m m}>0$ & $i+\frac{1}{2}$ & $i-\frac{1}{2}$ & $\Delta q_{y}^{m m}>0$ & $j+\frac{1}{2}$ & $j-\frac{1}{2}$ & $\Delta q_{z}^{m m}>0$ & $k+\frac{1}{2}$ & $k-\frac{1}{2}$ \\
\hline$\Delta q_{x}^{m m}<0$ & $i-\frac{1}{2}$ & $i+\frac{1}{2}$ & $\Delta q_{y}^{m m}<0$ & $j-\frac{1}{2}$ & $j+\frac{1}{2}$ & $\Delta q_{z}^{m m}<0$ & $k-\frac{1}{2}$ & $k+\frac{1}{2}$ \\
\hline
\end{tabular}

are obtained from the minmod differences in the corresponding coordinate direction. The indices of the second point to be checked for a maximum $\left(\mathbf{C}_{2}^{\max }\right)$ and for a minimum $\left(\mathbf{C}_{2}^{\text {min }}\right)$, respectively, follow from

\begin{tabular}{ccc} 
& $\mathbf{C}_{2}^{\max }$ & $\mathbf{C}_{2}^{\min }$ \\
\hline$\left|\Delta q_{x}\right|<\min \left(\left|\Delta q_{y}\right|,\left|\Delta q_{z}\right|\right)$ & imin,jmax, kmax & imax,jmin, kmin \\
$\left|\Delta q_{y}\right|<\min \left(\left|\Delta q_{x}\right|,\left|\Delta q_{z}\right|\right)$ & imax,jmin, kmax & imin, jmax, kmin \\
$\left|\Delta q_{z}\right|<\min \left(\left|\Delta q_{x}\right|,\left|\Delta q_{y}\right|\right)$ & imax,jmax,kmin & imin,jmin, kmax
\end{tabular}.

It can be summarized, that from the eight corners of a $3 \mathrm{D}$ volume two corners $\left(\mathbf{C}_{1}^{\max }\right.$ and $\left.\mathbf{C}_{2}^{\max }\right)$ have to be checked for a local maximum and two corners $\left(\mathbf{C}_{1}^{\min }\right.$ and $\left.\mathbf{C}_{2}^{\min }\right)$ for a local minimum.

To illustrate the required check an example for a local maximum is given in Fig. 6. We assume $\Delta q_{x}^{m m}=10, \Delta q_{y}^{m m}=8$, and $\Delta q_{z}^{m m}=2$ and it follows from Eqs. (59) and (60) that the corners $\mathbf{C}_{1}^{\max }$ located at $i+1 / 2, j+1 / 2, k+1 / 2$ and $\mathbf{C}_{2}^{\max }$ located at $i+1 / 2, j+1 / 2, k-1 / 2$ have to be checked for a local maximum. With $\alpha_{x}=\alpha_{y}=\alpha_{z}=2$

$$
q_{i \max , j \max , k \max }^{M L P}-q_{i, j, k}=20, \quad q_{i \max , j \max , k \min }^{M L P}-q_{i, j, k}=16
$$

is obtained from Eq. (57) for the corners $\mathbf{C}_{1}^{\max }$ and $\mathbf{C}_{2}^{\max }$, respectively. Both corners have to be checked to be a local maximum. Without additional information from neighbouring volumes it follows from Eq. (56) that

$$
Q_{i \max , j \max , k \max }^{\max }-q_{i, j, k} \geq 10, \quad Q_{i \max , j \max , k \min }^{\max }-q_{i, j, k} \geq 10 .
$$

These values are smaller than the MLP values at both corners and limitation 


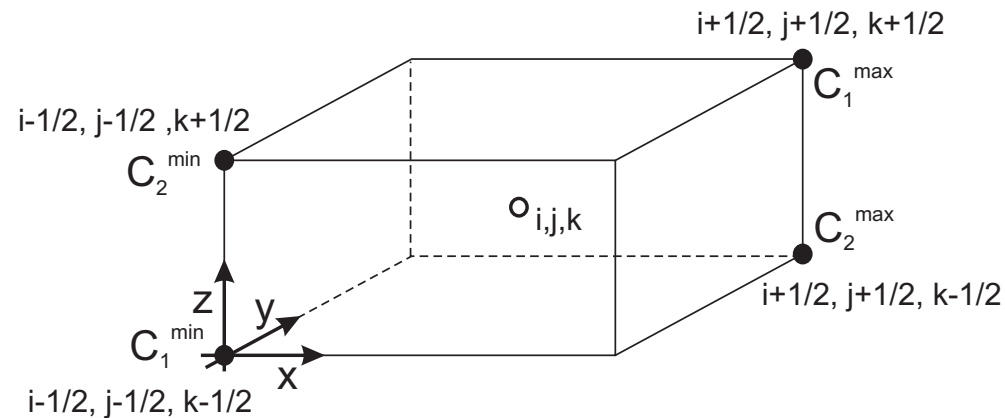

Figure 6: Volume $i, j, k$ with corners (indicated by $\bullet$ ) to be checked for a local maximum $\left(\mathbf{C}_{1}^{\max }\right.$ and $\left.\mathbf{C}_{2}^{\max }\right)$ or minimum $\left(\mathbf{C}_{1}^{\min }\right.$ and $\left.\mathbf{C}_{2}^{\min }\right)$ in case of $\Delta q_{x}^{m m}=10, \Delta q_{y}^{m m}=8$, and $\Delta q_{z}^{m m}=2$.

may be required. Due to the neglected diagonal values the inequality sign appears in Eq. (62) and the actual values may be higher. If, for example, there is a high value at the diagonal volume $i+1, j+1, k+1\left(q_{i+1, j+1, k+1}-q_{i, j, k}>20\right)$ it follows that $Q_{\text {imax } \max a x, k \max }-q_{i, j, k}>20$ and no MLP limitation is required at the corner $\mathbf{C}_{1}^{\max }$. At the same time corner $\mathbf{C}_{2}^{\max }$ still may require limiting.

It can be summarized, that two corners of a volume have to be checked for a maximum and two for a minimum. The remaining corners do not need to be checked.

Proof: Given $\left|\Delta q_{x}^{m m}\right| \geq\left|\Delta q_{y}^{m m}\right| \geq\left|\Delta q_{z}^{m m}\right|$ and using cell center values in the coordinate directions only $(i, j, k ; i \pm 1, j, k ; i, j \pm 1, k ; i, j, k \pm 1)$, the following limits for the eight maximum corner values are obtained from Eq. (56)

$$
\begin{aligned}
& Q_{i m a x, j \max , k+\kappa_{z} / 2}^{\max }-q_{i, j, k} \geq\left|\Delta q_{x}^{m m}\right|, \\
& Q_{i m a x}^{\max , j \min , k+\kappa_{z} / 2}-q_{i, j, k} \geq\left|\Delta q_{x}^{m m}\right|, \\
& Q_{i m i n}^{\max , j \max , k+\kappa_{z} / 2}-q_{i, j, k} \geq\left|\Delta q_{y}^{m m}\right|, \\
& Q_{i \min , j \min , k+\kappa_{z} / 2}^{\max }-q_{i, j, k} \geq\left|\Delta q_{z}^{m m}\right|
\end{aligned}
$$

with $\kappa_{z}= \pm 1$. The right hand sides follow from the definition of the minmod limiter where, e.g. in $x$-direction, $\left|q_{i+1, j, k}-q_{i, j, k}\right| \geq\left|\Delta q_{x}^{m m}\right|$ and $\mid q_{i, j, k}-$ $q_{i-1, j, k}|\geq| \Delta q_{x}^{m m} \mid$ are valid. To neglect a corner in the search for a local extremum requires, that the MLP criterium is satisfied even for maximum values 
of $\alpha$. Using Eq. (57) and $\alpha_{x}=\alpha_{y}=\alpha_{z}=2$

$$
\begin{aligned}
& q_{i m a x, j m a x, k+\kappa_{z} / 2}^{M L P}-q_{i, j, k}=\left|\Delta q_{x}^{m m}\right|+\left|\Delta q_{y}^{m m}\right| \pm\left|\Delta q_{z}^{m m}\right| \geq\left|\Delta q_{x}^{m m}\right|, \\
& q_{i m a x, j m i n, k+\kappa_{z} / 2}^{M L P}-q_{i, j, k}=\left|\Delta q_{x}^{m m}\right|-\left|\Delta q_{y}^{m m}\right| \pm\left|\Delta q_{z}^{m m}\right| \leq\left|\Delta q_{x}^{m m}\right|, \\
& q_{i m i n, j \max , k+\kappa_{z} / 2}^{M L P}-q_{i, j, k}=-\left|\Delta q_{x}^{m m}\right|+\left|\Delta q_{y}^{m m}\right| \pm\left|\Delta q_{z}^{m m}\right| \leq\left|\Delta q_{y}^{m m}\right|, \\
& q_{\text {imin }, j \min , k+\kappa_{z} / 2}^{M L P}-q_{i, j, k}=-\left|\Delta q_{x}^{m m}\right|-\left|\Delta q_{y}^{m m}\right| \pm\left|\Delta q_{z}^{m m}\right| \leq \mid \Delta q_{z}^{m m}(64)
\end{aligned}
$$

is obtained. Equations (63) and (64) are used to check if the MLP condition (58) is satisfied. It follows, that the first conditions from Eqs. (63) and (64) are not automatically fulfilled and have to be checked. This corresponds to the check of points $\mathbf{C}_{1}^{\max }$ and $\mathbf{C}_{2}^{\max }$. At the remaining corners the Eqs. (63) and (64) (lower three lines) show, that the MLP condition is always satisfied. The proof may be performed in the same way for a local minimum or different magnitudes of $\left|\Delta q_{x}^{m m}\right|,\left|\Delta q_{y}^{m m}\right|,\left|\Delta q_{z}^{m m}\right|$.

The fact that two corners have to be checked in 3D for a maximum/minimum complicates MLP. On one hand two independent checks have to be performed from which some minimum/maximum has to be taken. On the other hand the minus sign for one of the contributions to $q^{M L P}$ at corner $\mathbf{C}_{2}^{\max }$ (or the plus sign for one of the contributions to $q^{M L P}$ at corner $\mathbf{C}_{2}^{\min }$ ) causes problems. For example, if $-\alpha_{z}\left|\Delta q_{z}^{m m}\right|$ is a contribution to check corner $\mathbf{C}_{2}^{\max }$ to be a local maximum, then a reduction in $\alpha_{z}$ does not decrease this contribution to the maximum, but increases it. Therefore a simplified check is proposed

$$
\begin{aligned}
\alpha_{x}\left|\Delta q_{x}^{m m}\right|+\alpha_{y}\left|\Delta q_{y}^{m m}\right|+\alpha_{z}\left|\Delta q_{z}^{m m}\right| & \leq 2 \min \left(Q_{1}^{\max }-q_{i, j, k}, Q_{2}^{\max }-q_{i, j, k}+2 \mid \Delta q_{\min }^{m m}(665)\right. \\
-\alpha_{x}\left|\Delta q_{x}^{m m}\right|-\alpha_{y}\left|\Delta q_{y}^{m m}\right|-\alpha_{z}\left|\Delta q_{z}^{m m}\right| & \geq 2 \max \left(Q_{1}^{\min }-q_{i, j, k}, Q_{2}^{\min }-q_{i, j, k}-2 \mid \Delta q_{\min }^{m m}(6) 6\right)
\end{aligned}
$$

to keep the computational effort low. These equations follow from Eq. (58) by inserting Eq. (57) and changing the right hand sides to take the second corners $\mathbf{C}_{2}^{\max }$ and $\mathbf{C}_{2}^{\min }$ into account. Here $Q_{1}^{\max }$ and $Q_{2}^{\max }$ are maximum corner values at points $\mathbf{C}_{1}^{\max }$ and $\mathbf{C}_{2}^{\max }$, respectively, and $\left|\Delta q_{\min }^{m m}\right|=$ $\min \left(\left|\Delta q_{x}^{m m}\right|,\left|\Delta q_{y}^{m m}\right|,\left|\Delta q_{z}^{m m}\right|\right)$ is the smallest absolute change from the three coordinate directions. As in 2D, the checks for a maximum and minimum may 
be combined into a single MLP condition where

$$
\begin{aligned}
\alpha_{x}\left|\Delta q_{x}^{m m}\right|+\alpha_{y}\left|\Delta q_{y}^{m m}\right|+\alpha_{z}\left|\Delta q_{z}^{m m}\right| \leq & 2 \min \left(Q_{1}^{\max }-q_{i, j, k}, Q_{2}^{\max }-q_{i, j, k}+2\left|\Delta q_{\min }^{m m}\right|,\right. \\
& \left.q_{i, j, k}-Q_{1}^{\min }, q_{i, j, k}-Q_{2}^{\min }+2\left|\Delta q_{\min }^{m m}\right|\right) \\
= & \Delta q^{c}
\end{aligned}
$$

has to be satisfied. Again $\Delta q^{c}$ defines the limit (obtained from surrounding cell center values) for the reconstructed interface states.

There is another important difference between MLP in 2D and 3D which does not become clear from previous publications. In 2D the minmod limiter $\left(\alpha_{x}=\alpha_{y}=1\right)$ is a possible lower limit for MLP and thus prevents local extrema at corner points. Moreover, the minmod limiter still is in the second order TVD region. In 3D the MLP criteria may require $\alpha_{x}, \alpha_{y}, \alpha_{z}<1$.

Proof: Given is $\left|\Delta q_{x}^{m m}\right| \geq\left|\Delta q_{y}^{m m}\right| \geq\left|\Delta q_{z}^{m m}\right|$. If $\alpha_{x}=\alpha_{y}=\alpha_{z}=1$ is used for interface value reconstruction and if the corner with highest value $\mathbf{C}_{1}^{\max }$ is checked, it follows from the first line of Eq. (63)

$$
Q_{\text {imax }, j \max , k \max }^{\max }-q_{i, j, k}=\left|\Delta q_{x}^{m m}\right|
$$

as a lower limit, and from the first line of (64)

$$
q_{\text {imax }, \text { jmax }, \text { max }}^{M L P}-q_{i, j, k}=0.5\left|\Delta q_{x}^{m m}\right|+0.5\left|\Delta q_{y}^{m m}\right|+0.5\left|\Delta q_{z}^{m m}\right| .
$$

Thus with $\alpha_{x}=\alpha_{y}=\alpha_{z}=1$ the MLP condition

$$
0.5\left|\Delta q_{x}^{m m}\right|+0.5\left|\Delta q_{y}^{m m}\right|+0.5\left|\Delta q_{z}^{m m}\right| \leq\left|\Delta q_{x}^{m m}\right|
$$

is not satisfied if $\left|\Delta q_{y}^{m m}\right|+\left|\Delta q_{z}^{m m}\right|>\left|\Delta q_{x}^{m m}\right|$. In case of $\left|\Delta q_{x}^{m m}\right|=\left|\Delta q_{y}^{m m}\right|=$ $\left|\Delta q_{z}^{m m}\right|$ this condition is fulfilled for $\alpha_{x}=\alpha_{y}=\alpha_{z}=2 / 3$.

\subsection{MLP ${ }^{l d}$ for three-dimensional flow}

With the MLP condition (67) there is only one equation for three unknown parameters $\alpha_{x}, \alpha_{y}$, and $\alpha_{z}$. As to avoid excessive numerical dissipation, the upper limit is chosen in Eq. (67), corresponding to the equality sign

$$
\left|\Delta q^{M L P}\right|=\alpha_{x}\left|\Delta q_{x}^{m m}\right|+\alpha_{y}\left|\Delta q_{y}^{m m}\right|+\alpha_{z}\left|\Delta q_{z}^{m m}\right|=\Delta q^{c} .
$$




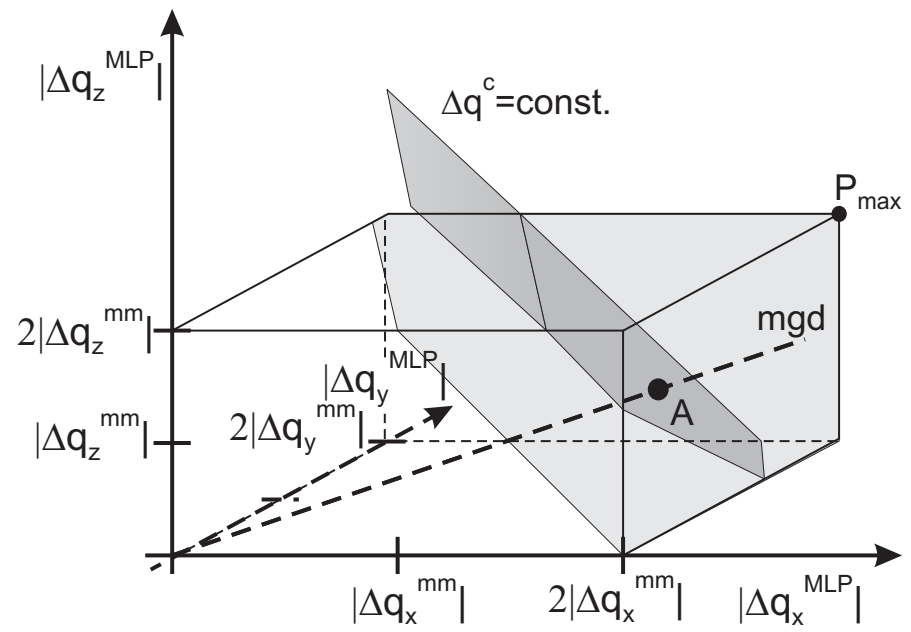

Figure 7: Realizable $\mathrm{MLP}^{l d}$ region (lightly shaded volume) in 3D. The dark shaded plane corresponds to $\Delta q^{c}=$ constant. The point of intersection of this plane with the mean gradient direction $(m g d)$, indicated with $\mathbf{A}$, defines the solution and thus $\alpha_{x}, \alpha_{y}$, and $\alpha_{z}$.

As proofed before, this condition may require $\alpha_{x}, \alpha_{y}, \alpha_{z}<1$. In principle $\alpha_{x}, \alpha_{y}, \alpha_{z} \in[2 / 3,2]$ could be used. However, as to achieve a better agreement between the mean flow direction and the gradient of the interface values, the requirements on $\alpha$ are released and $\alpha_{x}, \alpha_{y}, \alpha_{z} \in[0,2]$ is allowed in the present approach. This improves the convergence behaviour while the impact on the accuracy of the solution is very small. Again there is a lower limit for $\Delta q^{c}$ which does not allow $\alpha_{x}, \alpha_{y}$, and $\alpha_{z}$ to simultaneously take small values. In the same way Eq. (44) is derived in two-dimensional flow

$$
\Delta q_{\min }^{c}=2 \max \left(\left|\Delta q_{x}^{m m}\right|,\left|\Delta q_{y}^{m m}\right|,\left|\Delta q_{z}^{m m}\right|\right)
$$

follows in 3D, using direct neighbours of volume $i, j, k$ only. Figure 7 shows the possible MLP region for $r_{x}, r_{y}, r_{z}>0$ in $\left|\Delta q_{x}\right|-\left|\Delta q_{y}\right|-\left|\Delta q_{z}\right|$-space by the lightly shaded volume. As may be seen, the cuboid defined by $\alpha_{x}, \alpha_{y}, \alpha_{z} \in[0,2]$ is narrowed by the plane $\Delta q_{\min }^{c}=2\left|\Delta q_{x}^{m m}\right|$ (this is the lower limit in the present case) to a significantly smaller volume.

The MLP condition (71) defines a plane of constant $\Delta q^{c}$. If the change in one coordinate direction is frozen, the slope of this plane is minus one. While 
$\Delta q_{\min }^{c}$ is the lower limit, the upper one is given by $\Delta q_{\max }^{c}=2\left(\left|\Delta q_{x}^{m m}\right|+\right.$ $\left.\left|\Delta q_{y}^{m m}\right|+\left|\Delta q_{z}^{m m}\right|\right)$. The corresponding plane would cross the point $\mathbf{P}_{\max }=$ $\mathbf{P}\left(2\left|\Delta q_{x}^{m m}\right|, 2\left|\Delta q_{y}^{m m}\right|, 2\left|\Delta q_{z}^{m m}\right|\right)$ plotted in Fig. 7. Any $\Delta q^{c}$ higher than $\Delta q_{\max }^{c}$ results in $\alpha_{x}=\alpha_{y}=\alpha_{z}=2$. An example for a plane of constant $\Delta q^{c}$ is shown in Fig. 7 by the dark shaded area.

The determination of $\alpha_{x}, \alpha_{y}$ and $\alpha_{z}$ is based on the same criteria as in the two-dimensional case:

1. The MLP condition (67) has to be satisfied to achieve, that the interface values do not cause a local extremum at any of the corners of the volume.

2. Maximum values for $\alpha_{x}, \alpha_{y}$, and $\alpha_{z}$ are required by using $\left|\Delta q^{M L P}\right|=\Delta q^{c}$ (low diffusion).

3. The MLP interface values should have a gradient which corresponds to the mean gradient direction.

First step in the determination of $\alpha_{x}, \alpha_{y}$, and $\alpha_{z}$ is to calculate the coordinates of the point of intersection $\mathbf{A}$ between the line of the absolute mean gradient direction and the limiting plane $\left|\Delta q^{M L P}\right|=\Delta q^{c}=$ const. (see Fig. 7). The required mean gradient direction is obtained from second order central differences

$\left|\Delta \bar{q}_{x}\right|=\left|q_{i+1, j, k}-q_{i-1, j, k}\right|, \quad\left|\Delta \bar{q}_{y}\right|=\left|q_{i, j+1, k}-q_{i, j-1, k}\right|, \quad\left|\Delta \bar{q}_{z}\right|=\left|q_{i, j, k+1}-q_{i, j, k-1}\right|$

which are used to calculate the scaling factor

$$
f=\Delta q^{c} /\left(\left|\Delta \bar{q}_{x}\right|+\left|\Delta \bar{q}_{y}\right|+\left|\Delta \bar{q}_{z}\right|\right)
$$

from which the coordinates of the point of intersection $\mathbf{A}=\mathbf{A}\left(A_{x}, A_{y}, A_{z}\right)$

$$
A_{x}=f\left|\Delta \bar{q}_{x}\right|, \quad A_{y}=f\left|\Delta \bar{q}_{y}\right|, \quad A_{z}=f\left|\Delta \bar{q}_{z}\right|
$$

are obtained. The coordinates of $\mathbf{A}$ have to be checked to be below the upper boundary of the realizable MLP region

$$
A_{x} \leq 2\left|\Delta q_{x}^{m m}\right|, \quad A_{y} \leq 2\left|\Delta q_{y}^{m m}\right|, \quad A_{z} \leq 2\left|\Delta q_{z}^{m m}\right|
$$




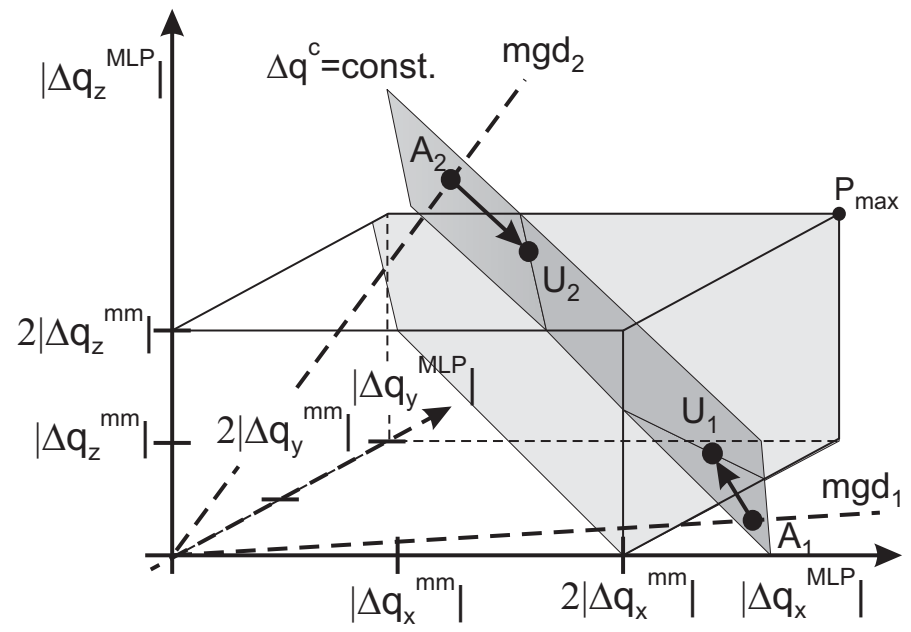

Figure 8: Realizable $\mathrm{MLP}^{l d}$ region (lightly shaded volume) in 3D. The dark shaded plane corresponds to $\Delta q^{c}=$ constant. The point of intersection $\left(\mathbf{A}_{1}\right.$ or $\left.\mathbf{A}_{2}\right)$ of this plane with the mean gradient direction $\left(m g d_{1}\right.$ or $\left.m g d_{2}\right)$ is located outside the MLP region and has to be shifted back to the MLP boundary $\left(\mathbf{U}_{1}\right.$ or $\left.\mathbf{U}_{2}\right)$.

(the lower limit is met automatically). In the following three cases are possible which are treated separately.

A: Point $\mathbf{A}$ is in the realizable MLP region and satisfies Eq. (76). In this case the point of intersection $\mathbf{A}=\mathbf{A}\left(A_{x}, A_{y}, A_{z}\right)=\mathbf{A}\left(\left|\Delta q_{x}^{M L P}\right|,\left|\Delta q_{y}^{M L P}\right|,\left|\Delta q_{z}^{M L P}\right|\right)$ is the final solution and the required $\alpha_{x}, \alpha_{y}$ and $\alpha_{z}$ values follow from

$\alpha_{x}=\left|\Delta q_{x}^{M L P}\right| /\left|\Delta q_{x}^{m m}\right|, \quad \alpha_{y}=\left|\Delta q_{y}^{M L P}\right| /\left|\Delta q_{y}^{m m}\right|, \quad \alpha_{z}=\left|\Delta q_{z}^{M L P}\right| /\left|\Delta q_{z}^{m m}\right|$.

A corresponding example is given by point $\mathbf{A}$ plotted in Fig. 7.

B: Point $\mathbf{A}$ is outside the realizable MLP region according to Eq. (76) in one coordinate direction only. Corresponding situations are shown for the points $\mathbf{A}_{1}$ and $\mathbf{A}_{2}$ in Fig. 8. In this case, one of the three conditions given above has to be skipped. As in two-dimensional flow, $\mathrm{MLP}^{l d}$ requires the first two conditions to be fulfilled. Because $\left|\Delta q^{M L P}\right|=\Delta q^{c}$ is maintained, the final solution has to be 
located on the plane $\Delta q^{c}=$ constant. The first condition requires a shift back to the outer MLP boundary. The third condition is violated and the gradient of the interface values differs from the mean gradient direction. However, in contrast to two-dimensional simulations, there is not a single solution for the shift back to the outer MLP boundary. Due to the intersection of the plane $\Delta q^{c}=$ const. with the outer MLP boundary, a line with slope minus one is created (see e.g. the line crossing point $\mathbf{U}_{1}$ in Fig. 8). Any point on this line is a valid solution with respect to the first two conditions, given above. The question is now, which point on the line should be taken? The most obvious choice would be the point, which causes the smallest change with respect to the mean gradient direction. Because this solution is relatively costly, a more simple approach is taken, which comes close to it: The solution point $\mathbf{U}$ is defined by the smallest distance from $\mathbf{A}$ to $\mathbf{U}$ ( $\mathbf{U}$ is located on the line of intersection on the outer MLP boundary). In practice such a shift from $\mathbf{A}$ to $\mathbf{U}$ may be realized by simple measures:

1. The distance $h_{i}=\max \left(A_{i}-2\left|\Delta q_{i}^{m m}\right|, 0\right)$ to the outer boundary is calculated for all coordinate directions $i=x, y, z$. It is checked, if $A_{i}$ is outside the outer MLP boundary $\left(h_{i}>0\right)$.

2. If $h_{i}>0$ in one coordinate direction only:

(a) The $i$-coordinate of $\mathbf{U}=\mathbf{U}\left(U_{x}, U_{y}, U_{z}\right)$ is calculated by $U_{i}=A_{i}-$ $h_{i}$ to lie exactly on the plane of the outer MLP boundary with $\left|\Delta q_{i}^{M L P}\right|=2\left|\Delta q_{i}^{m m}\right|$.

(b) The remaining two coordinates $U_{j}, j=x, y, z$ and $j \neq i$ are obtained from $U_{j}=A_{j}+h_{i} / 2$.

In this way $\Delta q^{c}$ is kept constant $\left(\Delta q_{\mathbf{U}}^{c}=\Delta q_{\mathbf{A}}^{c}\right)$. It is simple to show, that a move on a plane with $x+y+z=$ const. by $-h_{i}$ in one coordinate direction and by $h_{i} / 2$ in the remaining two directions minimizes the distance between both points on the plane (the proof is not given but easy to obtain). Next it has to be checked, if the coordinates of $\mathbf{U}$ are inside the outer MLP boundary. Two cases have to be distinguished. 
B1: Point $\mathbf{U}$ is within the realizable $M L P$ region according to Eq. (76). In this case $\mathbf{U}$ is the final solution and defines the required $\alpha_{i}$ values (see e.g. $\mathbf{U}_{1}$ or $\mathbf{U}_{2}$ in Fig. 8).

B2: Point $\mathbf{U}$ is outside the realizable MLP region according to Eq. (76). The shift from $\mathbf{A}$ to $\mathbf{U}$ has caused a violation of the outer MLP limit in one of the $U_{j}, j=x, y, z$ and $j \neq i$ coordinates and another shift is required. Because $U_{i}$ already is on the outer MLP $i$-boundary and because only one $U_{j}$ may be located outside, the second shift is a simple move from $\mathbf{U}=\mathbf{U}\left(U_{x}, U_{y}, U_{z}\right)$ along a line with slope minus one to $\mathbf{S}=\mathbf{S}\left(S_{x}, S_{y}, S_{z}\right)$ as shown on the left side of Fig. 9. It is achieved by:

1. The distances $l_{j}=\max \left(U_{j}-2\left|\Delta q_{j}^{m m}\right|, 0\right)$ to the outer $j$-boundaries are calculated for the coordinate directions $j=x, y, z$ and $j \neq i$. It is checked, which $U_{j}$ is outside the outer MLP boundary $\left(l_{j}>0\right)$.

2. If $l_{j}>0$ :

(a) The $i$-coordinate remains constant because it already is located on the outer boundary $\left(S_{i}=U_{i}\right)$. The $j$-coordinate of $\mathbf{S}$ is calculated by $S_{j}=U_{j}-l_{j}$ to lie exactly on the plane of the outer MLP $j$-boundary. Thus $\mathbf{S}$ is located on one of the three outer edges of the MLP region.

(b) The remaining coordinate $U_{k}, k=x, y, z$ and $k \neq i, k \neq j$ is calculated by $S_{k}=U_{k}+l_{j}$.

$\mathbf{S}$ is the final solution and defines the required $\alpha_{i}$ values. An example for this procedure is given on the left side of Fig. 9.

C: Point $\mathbf{A}$ is outside the realizable MLP region according to Eq. (76) in two coordinate directions. In this case two shifts (from $\mathbf{A}$ to $\mathbf{U}$ and from $\mathbf{U}$ to $\mathbf{S}$ ) have to be performed, because after the first shift, a final solution is impossible.

Proof: Given is a point A with $A_{i}>2\left|\Delta q_{i}^{m m}\right|$ in two coordinate directions $i=x, y, z$. If $A_{i}>2\left|\Delta q_{i}^{m m}\right|$ point $\mathbf{U}$ is calculated by $U_{i}=A_{i}-h_{i}=2\left|\Delta q_{i}^{m m}\right|$. 

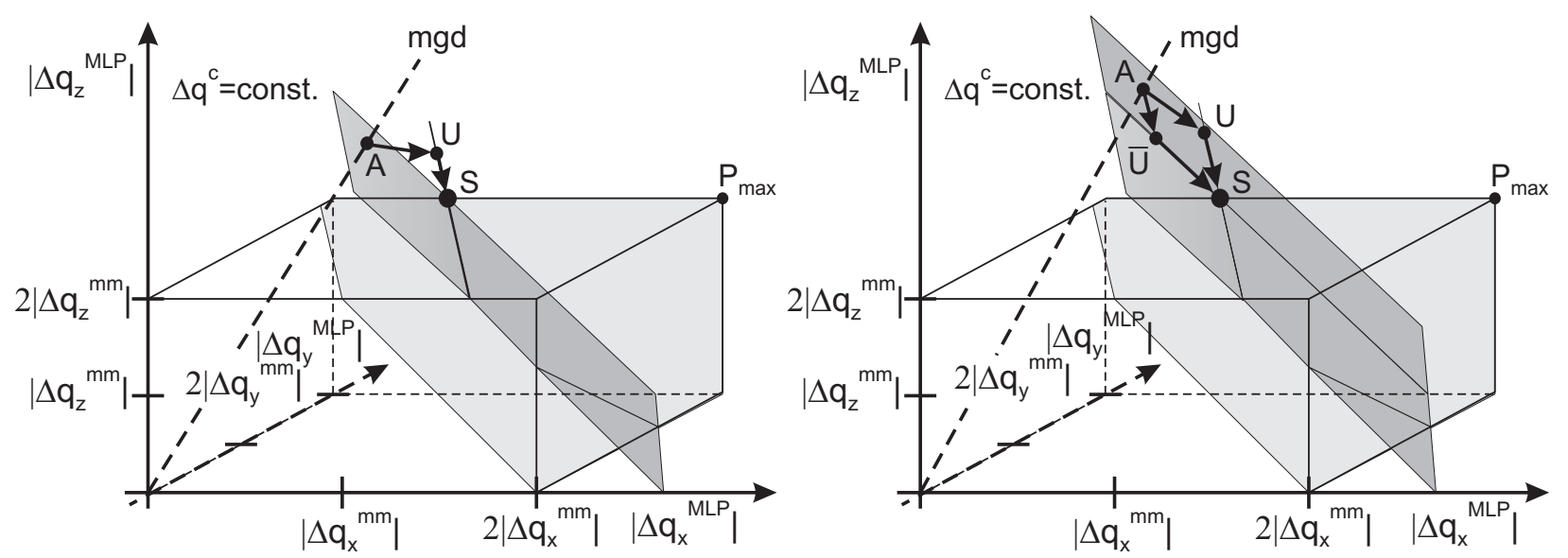

Figure 9: $\mathrm{MLP}^{l d}$ regions (lightly shaded volumes) in 3D. The dark shaded planes correspond to $\Delta q^{c}=$ constant. The point of intersection (A) of this plane with the mean gradient direction $(m g d)$ is located outside the MLP region in one coordinate direction only (left side), in two coordinate directions (right side). First shifts to $\mathbf{U}$ (or $\overline{\mathbf{U}}$ in the right figure) are performed. These points are still outside the MLP region and have to be shifted a second time to the final position $\mathbf{S}$.

The remaining coordinates of $\mathbf{U}$ are obtained from $U_{j}=A_{j}+h_{i} / 2$ for $j=x, y, z$ and $j \neq i$. Because one of the $A_{j}$ with $j \neq i$ already exceeds the outer MLP limit, this remains valid if $h_{i} / 2>0$ is added.

Thus two shifts are necessary, which are performed in the same way as described in $B$ and $B 2$. Because A exceeds the outer MLP boundary in two coordinate directions, these shifts may be performed in two ways, depending on which shift is performed first (A-U-S or $\mathbf{A}-\overline{\mathbf{U}}-\mathbf{S}$, as shown on the right side of Fig. 9). Irrespectively from which direction is taken first, the final solution points $\mathbf{S}$ are identical.

Proof: Given is point $\mathbf{A}=\mathbf{A}\left(A_{x}, A_{y}, A_{z}\right)$ with $\left|\Delta q_{i}\right|>2\left|\Delta q_{i}^{m m}\right|$ in two coordinate directions $i=x, y, z$. If $A_{i}$ exceeds the outer MLP boundary, point $\mathbf{U}$ is calculated as described above. A second shift in $j$-direction $(j \neq i)$ is performed and the final coordinates of the solution point $\mathbf{S}=\mathbf{S}\left(S_{x}, S_{y}, S_{z}\right)$ are $S_{i}=2\left|\Delta q_{i}^{m m}\right|, S_{j}=2\left|\Delta q_{j}^{m m}\right|$ and $S_{k}=A_{i}+A_{j}+A_{k}-2\left(\Delta q_{i}^{m m}\left|+\Delta q_{j}^{m m}\right|\right)$, with $i, j, k=x, y, z$ and $i \neq j \neq k \neq i$. The coordinates of $\mathbf{S}$ are independent 
from the order in which the shifts in $i$ - and $j$-direction are performed. Thus, the same result is obtained for $i$ being the first and $j$ the second shift, or reverse.

The last possibility, that the coordinates of the point of intersection $\mathbf{A}$ exceed the outer MLP boundary in all coordinate directions is of no importance.

Proof: If there is a point $\mathbf{A}$ with $\left|\Delta q_{i}\right|>2\left|\Delta q_{i}^{m m}\right|$ in all coordinate directions $i=x, y, z$, then $\Delta q_{\mathbf{A}}^{c}>\Delta q_{\max }^{c}$. In this case there is no interaction of the plane $\Delta q_{\mathbf{A}}^{c}=$ const. with the MLP region and the MLP criteria is not violated.

\section{$\operatorname{MLP}^{l d}$ procedure for the calculation of $\alpha_{x}, \alpha_{y}, \alpha_{z} \in[0,2]$ in three-} dimensional flows

For $i=x, y, z$ calculate $\left|\Delta q_{i}^{m m}\right|$ from Eq. (28), $\Delta q^{c}$ from the right part of Eq. (67), and the coordinates $A_{i}$ of point $\mathbf{A}$ from Eq. (75) and set $\alpha_{i}=2$.

$$
\begin{aligned}
& \text { if } \left.\left(2\left(\left|\Delta q_{x}^{m m}\right|+\left|\Delta q_{y}^{m m}\right|\right)+\left|\Delta q_{z}^{m m}\right|\right)>\Delta q^{c}\right) \text { then } \\
& \begin{aligned}
\text { do for } i & =x, y, z \\
h_{i} & =\max \left(A_{i}-2\left|\Delta q_{i}^{m m}\right|, 0\right) \\
U_{i} & =A_{i}-h_{i}, \quad U_{j}=A_{j}+h_{i} / 2 \text { for } j=x, y, z \text { and } j \neq i \\
f & =h_{i} /\left(h_{i}+\epsilon\right) \text { with } \epsilon \text { is a small number } \\
l & =f\left[\max \left(A_{j}-2\left|\Delta q_{j}^{m m}\right|, 0\right)-\max \left(A_{k}-2\left|\Delta q_{k}^{m m}\right|, 0\right)\right] \\
& j, k=x, y, z \text { and } j, k \neq i \text { and } j \neq k \\
S_{i} & =U_{i}, \quad S_{j}=U_{j}-l, \quad S_{k}=U_{k}+l \\
\alpha_{n} & =S_{n} /\left|\Delta q_{n}^{m m}\right|, A_{n}=S_{n} \text { for } n=x, y, z \\
\text { end do } &
\end{aligned} \\
& \text { end if }
\end{aligned}
$$

This formulation avoids numerous if-else constructions and is easy to program. The obtained $\alpha_{x}, \alpha_{y}$, and $\alpha_{z}$ values can be used in Eq. (23) in combination with any higher order approach for $\beta$. An advantage of MLP and MLP $l d$ is, that no free parameters are required. Moreover, the described MLP $^{l d}$ version for three-dimensional flows passes over in the two-dimensional version, if the 


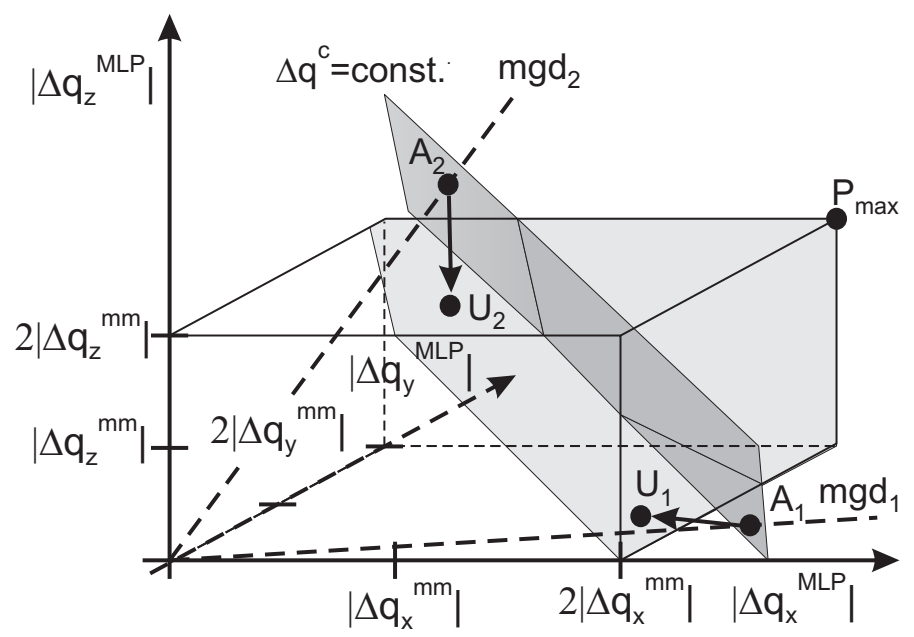

Figure 10: MLP region (lightly shaded volume) for the version of Kim et al. [1] using $\alpha_{x}, \alpha_{y}, \alpha_{z} \in[0,2]$ in 3D. The dark shaded plane corresponds to $\Delta q^{c}=$ constant. The point of intersection $\left(\mathbf{A}_{1}\right.$ or $\left.\mathbf{A}_{2}\right)$ of this area with the mean gradient directions is located outside the MLP region. It is shifted back to the outer MLP boundary $\left(\mathbf{U}_{1}\right.$ or $\mathbf{U}_{2}$ ) by moves in the corresponding coordinate directions.

gradient in the third direction becomes zero.

\subsection{MLP of Kim et al. [1] in 3D}

The 3D version of the MLP from Kim et al. [1] is nearly identical to the procedure in two-dimensional flow. The newly developed MLP ${ }^{l d}$ version and the original MLP are identical, as long as the point of intersection between the mean gradient direction and the gradient of the interface values $\mathbf{A}$ is within the possible MLP region. If this is not the case, point $\mathbf{A}$ has to be shifted back to the outer MLP boundary. In the version of Kim et al. this is done without keeping $\Delta q^{c}$ constant. Instead, simple shifts along the different coordinate directions are performed, as shown in Fig. 10. The points $\mathbf{A}_{1}$ and $\mathbf{A}_{2}$ are located outside the MLP region and are vertically or horizontally shifted back to the outer MLP boundary, respectively. As in $2 \mathrm{D}$, these measures are relatively easy to achieve. On the other hand, the problems explained for the 2D case remain valid in 3D. If the point of intersection $\mathbf{A}$ is outside the MLP region, $\Delta q^{c}$ is reduced beyond 
the limit required to avoid a local extremum. Additionally, MLP limitations may take place, where no limitation is needed.

\section{AUSM $^{+}$-up flux vector splitting}

For calculation of the inviscid fluxes through a cell interface the $\mathrm{AUSM}^{+}$-up flux vector splitting scheme of Liou [16] is used. The required primitive left $(L)$ and right $(R)$ interface variables are calculated as described in Sect. 3 to 5 with accuracies up to fifth order. According to Liou the inviscid flux vector $\mathbf{f}_{1 / 2}$ through an interface is splitted by

$$
\mathbf{f}_{1 / 2}=\dot{m}_{1 / 2} \boldsymbol{\Psi}_{L / R}+\mathbf{p}_{1 / 2}
$$

into a convective and a pressure flux. Based on the left and right interface values of the enthalpy $h_{L, R}$ and the ratio of integral specific heat capacities $\bar{\gamma}_{L, R}$ the critical speed of sound $a_{L, R}^{*}$ is calculated by

$$
a_{L, R}^{*}=\sqrt{2 \frac{\bar{\gamma}_{L, R}-1}{\bar{\gamma}_{L, R}+1} H_{L, R}^{n}} .
$$

Because multi-dimensional flows are treated, different critical speeds of sound are used for the different coordinate directions due to total enthalpies $H_{L, R}^{n}=$ $h_{L, R}+0.5 q_{L, R}^{2}$, which differ due to different interface velocities

$$
q_{L, R}=n_{x} u_{L, R}+n_{y} v_{L, R}+n_{z} w_{L, R} .
$$

In the last equation $\mathbf{n}=\left(n_{x}, n_{y}, n_{z}\right)$ is the unit normal vector for a cell interface. In this way a physically correct splitting between subsonic and supersonic flow is obtained at any cell interface. From these values an interface acoustic speed

$$
a_{1 / 2}=\min \left[\frac{a_{L}^{* 2}}{\max \left(a_{L}^{*}, q_{L}\right)}, \frac{a_{R}^{* 2}}{\max \left(a_{R}^{*},-q_{R}\right)}\right]
$$

is determined and subsequently the left and right state Mach numbers $M_{L, R}=$ $u_{L, R} / a_{1 / 2}$ as well as an averaged Mach number $\bar{M}^{2}=0.5\left(u_{L}^{2}+u_{R}^{2}\right) / a_{1 / 2}^{2}$. Next 
the split Mach numbers $M^{+}=M^{+}\left(M_{L}\right), M^{-}=M^{-}\left(M_{R}\right)$ and the split pressures $P^{+}=P^{+}\left(M_{L}\right), P^{-}=P^{-}\left(M_{R}\right)$ are calculated using

$$
\begin{aligned}
M^{ \pm}\left(M_{L}, M_{R}\right) & = \pm \frac{1}{4}(M \pm 1)^{2} \pm \frac{1}{8}\left(M^{2}-1\right)^{2} & |M|<1, & \\
& =\frac{1}{2}(M \pm|M|) & |M| \geq 1, & \text { (83) } \\
P^{ \pm}\left(M_{L}, M_{R}\right) & =\frac{1}{4}(M \pm 1)^{2}(2 \mp M) \pm \alpha M\left(M^{2}-1\right)^{2} & & |M|<1, \\
& =\frac{1}{2}(1 \pm \operatorname{sgn}(M) \cdot 1) & & |M| \geq 1(84)
\end{aligned}
$$

with $\alpha=3 / 16\left(-4+5 f_{a}^{2}\right)$. Finally the averaged Mach number and the averaged pressure

$$
\begin{aligned}
M_{1 / 2} & =M^{+}+M^{-}-K_{p} / f_{a} \max \left(1-\sigma \bar{M}^{2}, 0\right)\left(p_{R}-p_{L}\right) /\left(\rho_{1 / 2} a_{1 / 2}^{2}\right),(85) \\
p_{1 / 2} & =P^{+} p_{L}+P^{-} p_{R}-K_{u} P^{+} P^{-}\left(\rho_{L}+\rho_{R}\right) f_{a} a_{1 / 2}\left(u_{R}-u_{L}\right)
\end{aligned}
$$

are obtained with $f_{a}=M_{o}\left(2-M_{o}\right), M_{o}^{2}=\min \left(1, \max \left(\bar{M}^{2}, M_{\infty}^{2}\right)\right), \rho_{1 / 2}=$ $\left(\rho_{L}+\rho_{R}\right) / 2$ and the model constants $\sigma=1, K_{u}, K_{p} \in[0,1]$. Based on the averaged Mach number the mass fluxes follow from

$$
\begin{array}{ll}
\dot{m}_{1 / 2}=a_{1 / 2} M_{1 / 2} \rho_{L} & M_{1 / 2}>0, \\
\dot{m}_{1 / 2}=a_{1 / 2} M_{1 / 2} \rho_{R} & \text { otherwise, }
\end{array}
$$

and the total inviscid flux from

$$
\begin{array}{ll}
\mathbf{f}_{1 / 2}=\dot{m}_{1 / 2} \boldsymbol{\Psi}_{L}+\mathbf{p}_{1 / 2} & \dot{m}_{1 / 2}>0 \\
\mathbf{f}_{1 / 2}=\dot{m}_{1 / 2} \boldsymbol{\Psi}_{R}+\mathbf{p}_{1 / 2} & \text { otherwise }
\end{array}
$$

with $\boldsymbol{\Psi}=\left[1, u, v, w, H, q, \omega, Y_{1}, Y_{2}, \cdots, Y_{N_{k}-1}\right]$ and $\mathbf{p}_{1 / 2}=p_{1 / 2}\left[0, n_{x}, n_{y}, n_{z}, 0, \cdots, 0\right]$. For the supersonic test cases $M_{\infty}=1$ is chosen. The model constants $K_{u}$ and $K_{p}$ have an influence on the convergence behaviour and are chosen to be $K_{u}=K_{p}=0.25$.

\section{Results and Discussion}

To demonstrate the improvements in accuracy, stability, and convergence which are achieved by MLP and in particular the higher order MLP versions, a 
number of laminar and turbulent test cases are investigated. In addition to the limiter functions described above, the second order central discretization (with second and fourth order artificial viscosity) of Jameson et al. [26] is included in the study. With exception of the central difference scheme, all approaches use the $\mathrm{AUSM}^{+}$-up flux vector splitting of Liou [16] to calculate the inviscid fluxes at the cell interfaces.

\subsection{Two-dimensional Turbulent Flow Over a Backward Facing Step}

The first test case considered is a turbulent Mach 2 flow over a backward facing step, which has been investigated experimentally by McDaniels et al. [27]. The channel has a length of $45 \mathrm{~mm}$, a step height of $3.18 \mathrm{~mm}$, and, after the step, a channel height of $21.29 \mathrm{~mm}$. The computational grid uses two blocks with $112 \cdot 80$ and $144 \cdot 112$ volumes, respectively. It is extremely fine in the near wall regions and achieves $y^{+}$-values below one, required for the lowReynolds number $q-\omega$ turbulence model [17]. The cell aspect ratio is up to 850 near solid walls. Inlet profiles for the simulation have been calculated with the same code to match the experimentally measured boundary layer thickness of $\delta=1.45 \mathrm{~mm}$ at the $x=0$ location. Figure 11 shows the calculated pressure distribution using the fourth order $\mathrm{MLP}^{l d}$ scheme. A good agreement between simulation and experiment has been obtained using the second order central discretization with artificial viscosity [28]. The differences to the results of the present higher order flux vector splitting schemes with and without MLP are small. This is probably due to the very fine grid for a channel of $45 \mathrm{~mm}$ length and $21 \mathrm{~mm}$ height only. For steady state simulations, the improvements achieved by the fourth/fifth order discretization (in contrast to a low diffusive second order discretization) are limited to regions where the behaviour of the flow variables is non-linear. In the present case such a region is the recirculation zone downstream of the backward facing step. Figure 12 shows vertical profiles of the velocity component $u$ (left side) and of the turbulence variable $q(q=\sqrt{k}$, $k$ - turbulent kinetic energy, right side) at the position $x=5 \mathrm{~mm}$. Both figures show results using the 4 th order $\mathrm{MLP}^{l d}$ scheme and the second order van Leer 
MLP $^{l d}$ scheme on two grids: the standard grid (fine) and a coarse grid, where every second grid point in both coordinate directions is neglected. While in the smooth parts of the flow field the results are very close together, differences occur in the recirculation zone. The comparison with simulations using a coarse grid shows, that the fourth order coarse grid simulation is located right between the corresponding second order coarse and second order fine grid simulation. Thus a grid refinement by a factor of approximately 1.5 is required (in every coordinate direction) to achieve the same results with the second order van Leer $\mathrm{MLP}^{l d}$ scheme as with the fourth $\mathrm{MLP}^{l d}$ discretization (in strongly non-linear regions). Because the additional effort in $\mathrm{CPU}$ time for the fourth/fifth order scheme is negligible, the higher order schemes are recommended.

Convergence histories for this test case are plotted in Fig. 13 for a number of different discretizations. On the left side, averaged, normalized, absolute density residuals are plotted versus the number of iterations. On the right side, corresponding turbulence residuals (for $q$ ) are shown. As may be seen from both figures, convergence using the standard van Leer $(\mathrm{vL})$ limiter stalls after a residual reduction of two orders of magnitude. All other second order schemes converge at similar rates, as far as the density residual is concerned. Besides the van Leer MLP scheme of Kim et al. [1] with $\alpha_{x}, \alpha_{y} \in[0,2]$, this includes the newly proposed $\mathrm{MLP}^{l d}$ scheme with $\alpha_{x}, \alpha_{y} \in[1,2]$ and $\alpha_{x}, \alpha_{y} \in[0,2]$, a minmod limiter based scheme, and the central difference scheme. Concerning the turbulence residuals, there is a stall in convergence for the MLP ${ }^{l d}$ scheme using $\alpha_{x}, \alpha_{y} \in[1,2]$ after three orders of magnitude. This scheme is the least dissipative of the three MLP schemes investigated. Obviously more dissipation is needed, which enables the MLP scheme of Kim et al. and the MLP ${ }^{l d}$ scheme (both with $\alpha_{x}, \alpha_{y} \in[0,2]$ ) to converge. Concerning the quality of the obtained results, the differences between the three MLP schemes are minimal. In case of $\operatorname{MLP}^{l d}$ with $\alpha_{x}, \alpha_{y} \in[1,2]$ the stall of convergence is caused by a few volumes only, located directly at the oblique shock wave. Based on this experience it is recommended, to use the somewhat more dissipative version $\mathrm{MLP}^{l d}$ with $\alpha_{x}, \alpha_{y} \in[0,2]$. 
On the left side of Fig. 14 convergence histories for the higher order schemes are plotted (one second order result is shown for comparison). While the third and fourth order $\mathrm{MLP}^{l d}$ schemes converge at nearly the same rate as the corresponding second order scheme, the fifth order discretization causes a stall after a residual reduction of two orders of magnitude. The stronger upwind character of the fourth order scheme could be an explanation for its better behavior in this supersonic test case. Figure 14 (right side) shows normalized pressure profiles along the channel height at $x=40 \mathrm{~mm}$. This figure is an extension of the region $9.6 \mathrm{~mm} \leq y \leq 11.2 \mathrm{~mm}$ and thus a cut through the pressure increase caused by the reattachment shock (see Fig. 11). As expected, the shock is smeared the most by the minmod limiter, followed by the central discretization scheme. The good convergence properties of both schemes are paid for by an increased numerical dissipation. The remaining MLP schemes are relatively close together. Thus the higher accuracy of the MLP schemes is combined with convergence rates, which are comparable to more dissipative approaches.

Finally the newly proposed MLP ${ }^{l d}$ scheme is compared with the MLP approach of Kim et al. on a sequence of increasingly coarser grids (both versions are based on $\left.\alpha_{x}, \alpha_{y} \in[0,2]\right)$. Grid 1 is the original fine grid and the grids 2 to 4 are always obtained by neglecting any second grid point in both coordinate directions. The comparison is performed using the fourth order upwind biased discretization. Figure 15 shows extensions of vertical pressure profiles through the reattachment shock at the positions $x=20 \mathrm{~mm}$ (left side) and $x=40$ $\mathrm{mm}$ (right side), respectively. On the fine grids, the differences between both approaches are relatively small. However, they increase with decreasing grid size. As may be seen from both figures, MLP ${ }^{l d}$ is always closer to the solution on the next finer grid. Due to the smaller amount of added viscosity the shock resolution improves.

\subsection{Two-dimensional Turbulent Supersonic Hydrogen Mixing Channel}

This test case is based on experimental investigations performed at the University of Stuttgart [29]. Subject is a Mach 2 supersonic mixing channel, where 
hydrogen is injected through the blunt end of a strut injector, located at the channel axis. The simulated channel length is about $500 \mathrm{~mm}$, the channel height is $35.4 \mathrm{~mm}$. The simulation starts with sonic inlet conditions at the nozzle throat, where the supersonic flow is created in the experiment. The hydrogen nozzle inside the strut is included in the computational grid. For turbulence closure, a low Reynolds number $q-\omega$ turbulence model is used. To achieve $y^{+}$-values smaller or around one, very fine grids are required at all near wall regions. Distances from the first cell centers to the walls are smaller than $0.5 \cdot 10^{-6} \mathrm{~m}$. A three block grid with $472 \cdot 32,432 \cdot 56$ and $672 \cdot 96$ volumes is used in these simulations. The cell aspect ratios reached in the boundary layer are higher than 2000. More details concerning this test case as well as previous simulations (using the central difference scheme only) may be found in Ref. [29]. Figure 16 shows calculated pressure contours for the second order central difference scheme (upper figure) and the fourth order MLP ${ }^{l d}$ scheme (lower figure). Up to the end of the shown channel part, the shock waves induced by the strut are reflected approximately nine times. Moreover, the shocks cross large density gradients due to the injected hydrogen at the channel axis. While the central difference scheme performed well in the previous test case, here the shocks get smeared strongly due to the multiple wall reflections. This smearing is caused by the added second and fourth order artificial viscosity [26] which is required to avoid oscillations at shock waves. Using $\mathrm{AUSM}^{+}$-up and the fourth order $\mathrm{MLP}^{l d}$ scheme, a much better shock resolution is obtained. Figure 17 shows wall static pressures along the channel length for different discretization approaches. Large differences between the $\mathrm{MLP}^{l d}$ schemes and the central difference scheme are observed. Moreover, the pressure level of the central difference scheme is significantly higher. The differences between the second and the fourth and fifth order $\mathrm{MLP}^{l d}$ schemes are much smaller. Nevertheless there is a clearly visible improvement achieved by the fourth and fifth order $\mathrm{MLP}^{l d}$ schemes, when compared to the second order MLP ${ }^{l d}$ discretization.

Even for this relatively complex test case with multiple shock reflexion, good convergence properties are obtained (not shown). More than five orders of 
magnitude residual reduction (for both density and turbulence residuals) are obtained with the fifth order $\mathrm{MLP}^{l d}$ scheme. This is more than one order of magnitude more than with the standard MLP scheme of Kim et al.

\subsection{Laminar Supersonic Flow over Intersecting Wedges}

The first three-dimensional test case is a laminar supersonic flow over two intersecting wedges. The corresponding experiment has been performed by Charwat and Redekeopp [30]. Figure 18 shows a sketch of the geometrical setup. In the present investigation, the corner flow is symmetric and both wedge angles are identical $\left(\alpha=\beta=12.2^{\circ}\right)$. The inflow Mach number is 3.17 and the Reynolds number is $6.73 \cdot 10^{6} 1 / \mathrm{m}$, respectively. Displacement effects and viscous-inviscid interaction can have an influence on the entire inviscid flow structure further downstream [31]. This test case is well suited to investigate three-dimensional flow separation, its interaction with shock waves, as well as shock resolution in inviscid parts of the flow field. Figure 19 shows calculated density contours at $x=25.4 \mathrm{~mm}$, corresponding to a Reynolds number of $1.63 \cdot 10^{5}$. As can be seen from this figure, the bow shocks caused by the wedges do not intersect but are joined by a third shock [30]. The internal flow field includes two strong, curved, embedded (inner) shocks, which terminate at the wedges surfaces. There is a considerable extension of the shock disturbed boundary layer and the disturbances laterally spread far beyond the locations of the embedded shock waves. From the triple points two slip surfaces start in the direction of the corner. As will be seen later, a special feature of the laminar intersecting wedge flow is the curvature of the inner shocks, which cause expansion fans, starting from the triple points. Besides the numerically obtained density distribution, Fig. 19 additionally shows measured locations of the two wedge shocks, the corner shock, and the embedded shocks (indicated by circles) as well as the slip surfaces (shear layers, indicated by squares). The experimental structures are determined from Pitot pressure survey mappings. All experimental flow features are well predicted by the simulation.

The numerically simulated volume covers a region of $30 \mathrm{~mm} \cdot 60 \mathrm{~mm}$. 
$60 \mathrm{~mm}$ in $x$-, $y$ - and $z$-direction, respectively. At the position $x=25.4 \mathrm{~mm}$ experimental data are available and all results shown later refer to this axial position. The computational grid used in all simulations consists of $64 \cdot 128$ - 128 volumes in $x$-, $y$ - and $z$-direction, respectively. The density distribution given above has been obtained using the fourth order MLP ${ }^{l d}$ scheme. The corresponding wall surface pressure distribution at $x=25.4 \mathrm{~mm}$ is plotted in Fig. 20. Again, the overall agreement between simulation and experiment is quite good. Differences only occur in the outer region, where the inner shock reaches the wedge surface. There the experimental pressures are lower than in the simulation. In the experiment there is an expansion between $y / x=0.5$ and $y / x=0.9$ which is associated to turning the flow away from the corner. However, as shown in the paper of Charwat and Redekeopp [30], the appearance of such a large range for the expansion is strongly Mach number dependent. It does not appear in this way at Mach numbers lower $(M a=2.78)$ or higher $(M a$ $=3.64)$ than the one investigated. Thus, not too much importance is given to this discrepancy. Because only for the investigated Mach number detailed experimental data is available, this one has been chosen.

The increase in pressure at $0.2<y / x<0.4$ is not a "simple" shock [30], but exhibits considerable overshoots in both, up- and downstream direction. As noted by Charwat and Redekeopp [30], upstream of the shock the pressure is higher than the undisturbed two-dimensional pressure $\left(p / p_{0}>1\right)$ over a length more than twice the distance from the corner to the shock (from $y / x \approx 0.4$ up to $y / x>0.8)$. Downstream of the shock there is an overshoot followed by an expansion and a nearly constant pressure level very close to the wall $(y / x<0.18)[30]$. These features are well reproduced by the simulation. For more information concerning the physical details of this test case see Ref. [30].

In Fig. 21 convergence behaviours are compared for a number of different discretization techniques. On the left side density residuals are plotted for a selection of second order schemes. Again convergence stalls after a residual reduction by two orders of magnitude using the van Leer (vL) limiter. The most dissipative minmod limiter on the other hand, achieves the best results. 
MLP clearly improves the level of residual reduction: the version of Kim et al. $\left(\alpha_{x}, \alpha_{y}, \alpha_{z} \in[0,2]\right)$ achieves a reduction by more than four, the new MLP ${ }^{l d}$ version even by five and a half orders of magnitude. The level of convergence for $\mathrm{MLP}^{l d}$ is comparable to the much more dissipative central difference scheme with second and fourth order artificial viscosity. At the start of the simulations all schemes converge at nearly the same rate. On the right side of Fig. 21 convergence histories for the higher order MLP schemes are plotted. While Kim et al. $[9,1]$ reported better convergence levels for the high order schemes in many cases, this is not observed in the present study. As may be seen from the right figure, the level of residual reduction reduces from the second to the fourth, and from the fourth to the fifth order MLP ${ }^{l d}$ scheme. Nevertheless, the convergence behaviour of the fifth order scheme is still good and significantly better, than of the second order van Leer scheme without MLP. Moreover, the level of residual reduction achieved by the newly proposed MLP ${ }^{l d}$ scheme is somewhat better, than for the version of Kim et al. [1]. This is unexpected because $\Delta q^{M L P}=\Delta q^{c}$ is kept constant in case of $\mathrm{MLP}^{l d}$, independently from the mean gradient direction. Thus the scheme should be less dissipative. A possible explanation for this behaviour is, that, as described in Sect. 5.1, two corner points are checked for a maximum and two for a minimum in case of $\mathrm{MLP}^{l d}$, while only one point is checked in the version of Kim et al. [1].

To investigate influences of the grid resolution on the convergence behaviour, a second series of simulations has been performed, using a coarser grid with 64 . $64 \cdot 64$ volumes in $x$-, $y$ - and $z$-direction, respectively. Corresponding results are given in Fig. 22. There are only small differences caused by the grid. In case of the second order schemes (left side) the levels of residual reduction improve for the conventional van Leer limiter as well as for the MLP ${ }^{l d}$ van Leer scheme, compared to the finer grid. The version of Kim et al. achieves slightly worse results. In case of the higher order schemes (right side), convergence improves significantly for the fourth order $\mathrm{MLP}^{l d}$ discretization, while the level of convergence of the fifth order scheme gets a little bit worse. No general trend can be deduced from this study. 
The shock resolutions achieved by the different schemes are investigated next. Figure 23 (left side) shows normalized wall static pressures at $x=25.4$ $\mathrm{mm}$ as a function of $y / x$. Differences between the different approaches become visible in the (physically correct) overshoots in the up- and downstream region of the shock. The minmod limiter and the central difference scheme add the highest amount of numerical dissipation. The remaining discretization techniques are close together. On the right side of Fig. 23 results from higher order MLP ${ }^{l d}$ simulations are shown in an extension, which only covers the pressure increase by the shock wave. The circles correspond to cell centers of the computational grid. While the results from the fourth and fifth order schemes are very close together, their shock resolution is somewhat better than that of the second order $\mathrm{MLP}^{l d}$ scheme.

The pre- and post-shock overshoots observed in wall static pressure have to disappear, if the wedge shock is crossed in considerable distance from the corner. Figure 24 (left side) shows such a normalized static pressure profile at $x=25.4 \mathrm{~mm}$ and $z=58.1 \mathrm{~mm}$. Given is an extension of the region, where the wedge shock is located. No overshoots are observed in this figure from any of the second order discretization schemes. The higher order schemes (not shown in this figure) are very close to the second order MLP versions at this position.

Finally the newly proposed $\mathrm{MLP}^{l d}$ scheme is compared with the MLP approach of Kim et al. [1] on a sequence of increasingly coarser grids. A similar comparison has been given before for the 2D flow over a backward facing step. The fifth order discretization and $\alpha_{x}, \alpha_{y}, \alpha_{z} \in[0,2]$ is used for both MLP versions. Grid 1 is the original fine grid, for grid 2 any second grid point is neglected in the $y$ - and $z$ direction, and the grids 3 and 4 are always obtained from the next finer grid by neglecting any second grid point in all coordinate directions. Figure 24 (right side) shows an extension of the wall static pressure in the region where the shock wave reaches the wall. As in the two-dimensional case, on the fine grids the differences between both approaches are small. Reasons are the high order of the discretization and the relatively fine grids for the small physical domain. However, the differences increase with decreasing grid size. 
As before the $\mathrm{MLP}^{l d}$ solutions are closer to the solution on the next finer grid than the solution with the MLP version of Kim et al. [1].

\subsection{Turbulent Supersonic Flow over Intersecting Wedges}

Concerning the geometry and inflow conditions this test case is nearly identical to the previous one. Main difference is an increased Reynolds number of $6.85 \cdot 10^{7} 1 / \mathrm{m}$ which causes a turbulent flow field. The corresponding experiment has been performed by West and Korkegi [31]. Again, two symmetric intersecting wedges are the experimental basis as illustrated in Fig. 18. In this case the wedge angles are $\alpha=\beta=9.48^{\circ}$ and the inflow Mach number is 3. Figure 25 shows calculated density contours for the $x=87.6 \mathrm{~mm}$ position, corresponding to a Reynolds number of approximately $6 \cdot 10^{6}$. In accordance with the laminar case, two wedge shocks and a corner shock are obtained. In addition to the numerical results Fig. 25 also shows measured locations of the two wedge shocks, the corner shock, and the embedded shocks (indicated by circles) as well as the slip surfaces (shear layers, indicated by squares). The curved embedded shocks from the laminar case are straight in this turbulent flow field. As a consequence, the expansion fans, starting at the triple points, disappear. The shock structure now is essentially independent of viscous effects. Both shock structures and shear layers are well predicted by the simulation.

The numerical simulations of this test case cover a region of $100 \mathrm{~mm} \cdot 152.4$ $\mathrm{mm} \cdot 152.4 \mathrm{~mm}$ in $x$-, $y$ - and $z$-direction, respectively. At the position $x=$ $87.6 \mathrm{~mm}$ experimental data are available and all results shown later refer to this axial position. The computational grid used in all simulations consists of 100 . $164 \cdot 164$ volumes in $x$-, $y$ - and $z$-direction, respectively. The $q-\omega$ low-Reynolds number turbulence closure [17] is used, which requires very fine grids in the near wall region. With exception of the tip of the wedge, all $y^{+}$-values of near wall cell centers are below one. The density distribution shown above has been obtained using the fourth order MLP ${ }^{l d}$ scheme. The corresponding wall surface pressure distribution at $x=87.6 \mathrm{~mm}$ is plotted in Fig. 26. Again, the overall agreement between simulation and experiment is quite good. Because the 
shock structure is nearly independent from viscous effects, the differences between the numerical schemes becomes relatively small. In the vicinity of shock waves the discretization order is reduced and the behaviour of the fourth/fifth order schemes and the second order schemes approach each other. This becomes obvious on the left side of Fig. 27 where normalized wall static pressure distributions at the position $x=87.6 \mathrm{~mm}$ are plotted for a number of different discretization techniques. All results are very close together. On the right side of Fig. 27 an extension of the pressure profile at the position $x=87.6 \mathrm{~mm}$ and $z=148 \mathrm{~mm}$ is shown. These profiles confirm the small differences caused by the discretization techniques employed. Differences between a low dissipative second order scheme and the fourth/fifth order schemes are limited to regions, where the profiles of the flow variables have a non-linear behaviour. Figure 28 shows the velocity components $v$ (left side) and $w$ (right side) in dependence of the $y$-coordinate at $x=100 \mathrm{~mm}$ and $h=15 \mathrm{~mm}$ above the lower wall. Given are simulations using the second order van Leer $\mathrm{MLP}^{l d}$ scheme and the fifth order $\mathrm{MLP}^{l d}$ discretization on two grids. Fine is the standard grid and the coarse grid is obtained by neglecting any second grid point in all coordinate directions. The results are similar as for the backward facing step test case. In the non-linear parts the fifth order scheme achieves results on the coarse grid, which are right between the second order coarse grid and the second order fine grid simulation. Even if the improvements are small, there still is an improvement by using the higher order schemes for this steady state test case. Because the computational effort is nearly the same, the high order scheme is recommended.

Even if the pressure profiles are relatively close together, the convergence histories for the different schemes considerably differ. Figure 29 shows density (left side) and turbulence ( $q$ variable, right side) residuals over the number of iterations for the different discretization techniques. As before the standard van Leer limiter achieves a residual reduction by two orders of magnitude only. MLP significantly improves this behaviour. The second order van Leer MLP ${ }^{l d}$ scheme reduces the residual levels by four orders of magnitude. For the fifth order MLP ${ }^{l d}$ scheme, convergence is even better and nearly reaches that of 
the dissipative minmod limiter. The convergence behaviour for the turbulence residuals is comparable or even better than for the density. A very positive point for the higher order $\mathrm{MLP}^{l d}$ schemes is, that the turbulence equations, which often suffer from stability problems caused by stiffness, show no difference to the remaining flow variables. Thus $\mathrm{MLP}^{l d}$ has proved to work reliably even in cases with low-Reynolds number turbulence closure.

\subsection{Subsonic Model Rocket Combustor}

The final test case is an unsteady subsonic flow in a model rocket combustor. The corresponding experiment has been performed at the Pennsylvania State University [32]. It has been designed to characterize the wall heat transfer, which is an important issue in rocket combustor design. The combustor is axisymmetric and has a diameter of $38.1 \mathrm{~mm}$ and a length of $286 \mathrm{~mm}$. Two upstream preburners produce oxidizer-rich and fuel-rich gases, respectively. The combustor is operated at 5.42 $\mathrm{MPa}$ pressure. Details concerning geometry and operating conditions may be found in Ref. [32], details concerning the simulation in Ref. [33, 34]. In this paper, a comparison between second and fifth order discretizations will be given only.

As shown in Ref. [33], a steady RANS (Reynolds Averaged Navier-Stokes) simulation of this test case did not achieve satisfactory results. Thus, unsteady simulations (URANS) have been performed. Due to the unsteadiness of the test case, the differences between second order and the fourth/fifth order discretizations are much more pronounced. Combustion is described by finite-rate chemistry, based on the reaction mechanism of Ó Conaire [35]. This kinetic scheme is well suited for high pressure hydrogen combustion. For turbulence closure the $q-\omega$ low-Reynolds number turbulence model is used. In the experiment combustor wall temperatures have been measured. These temperatures are use in a least square fit to obtain the wall temperatures for the numerical simulation. The computational grid has about 215000 volumes and is strongly refined near the oxidizer post tip and at all near wall regions. The chosen timestep for the simulation is 0.1 microseconds. After 10 milliseconds, time 
averaging of the flow variables is started (one flow through time is about 8.3 milliseconds). Figure 30 shows averaged temperature fields for this combustor. The results shown in the upper figure are obtained with the $\mathrm{AUSM}^{+}$-up flux vector splitting and the second order van Albada limiter to reconstruct left and right cell interface states. Results shown in the lower figure are based on the same flux vector splitting, however, with interface states calculated with the fifth order $\mathrm{MLP}^{l d}$ reconstruction. As can be seen from the temperature plots (see Ref. [34] for other variables), there are large differences in the results caused by the different discretization techniques. Both location and size of the flame is strongly influenced by the chosen discretization technique despite the fact, that the computational grid already is extremely fine (for a axisymmetric two-dimensional simulation). This is in contrast to the steady state simulations shown before, where the improvements obtained using a fourth/fifth order scheme have been relatively small. The differences become visible in the calculated wall heat fluxes too, which are plotted in Fig. 31 together with the experimentally measured values. The increase in heat flux in the front part of the combustor as well as the maximum value are predicted very well by the fifth order simulation. Further downstream the decreasing heat flux is overpredicted by both discretization techniques compared to the experiment. However, the fifth order simulation achieves better results than the second order approach. Considering the complexity of this test case, the agreement between the fifth order $\mathrm{MLP}^{l d}$ simulation and the experiment is very good.

\section{Conclusions}

The multi-dimensional limiting process (MLP) of Kim et al. [1] in its threedimensional version has been analysed to understand, how the different flow directions interact during interface state reconstruction. To accomplish this, a different approach has been used, compared to the derivations in the original paper. It was found to be important, that the gradient of the limited interface values agrees well with the mean gradient direction of the corresponding 
variables. Based on these investigations, a modified MLP version (MLP ${ }^{l d}$ low diffusion) has been proposed, which achieves higher $\alpha$ values (and thus less diffusion) in cases, where strong differences between the mean gradient and the gradient of the limited interface values occur. This is the case in the vicinity of discontinuities. Diffusion is kept as low as possible while still satisfying the MLP condition, independently from the mean gradient direction. By this measure local extrema at the corners of a volume are avoided. For three-dimensional flow it was found, that two corners of a volume have to be checked for a maximum and two for a minimum. All described features are considered in MLP ${ }^{l d}$.

The original and the newly proposed MLP versions are used for the first time to simulate complex turbulent test cases with and without combustion. From these results it is concluded, that the convergence properties of the new version are at least comparable to the original MLP. In some cases it performed even better. It was found advantageous to use the range $\alpha_{x}, \alpha_{y}, \alpha_{z} \in[0,2]$ to improve the convergence behaviour while the impact on the numerical results is extremely small. If the computational grid becomes very fine, results from MLP ${ }^{l d}$ and the original MLP version of Kim et al. agree very well. On coarse grids however, $\mathrm{MLP}^{l d}$ is closer to the results obtained on a finer grid. Moreover, $\mathrm{MLP}^{l d}$ has proven to work in a stable manner with low-Reynolds number turbulence closures and combustion. As already observed by Kim et al. $[9,1]$, MLP stabilizes the numerical scheme. Thus it is an efficient and simple method to extend conventional second order schemes to higher accuracies and at the same time improve convergence. This is especially attractive for unsteady simulations.

\section{Acknowledgements}

Parts of this work have been performed within the framework of the research program SFB-TR 40, funded by the German Research Foundation (DFG). All simulations have been performed at the High Performance Computing Center Stuttgart (HLRS). The author wishs to thank the DFG and the HLRS for their 
support.

The author also wishs to thank M. Lempke for providing the numerical results of one test case.

\section{References}

[1] S.-H. Yoon, C. Kim, K.-H. Kim, Multi-dimensional limiting process for three-dimensional flow physics analyses, Journal of Computational Physics 227 (2008) 6001-6043.

[2] B. V. Leer, Towards the ultimate conservative difference scheme ii: Monotonicity and conservation combined in a second order scheme, Journal of Computational Physics 14 (1974) 361-370.

[3] A. Harten, High resolution schemes for hyperbolic conservation laws, Journal of Computational Physics 49 (1983) 357-393.

[4] M. Berger, M. J. Aftosmis, S. M. Murman, Analysis of slope limiters on irregular grids, Aiaa paper 2005-0490 (2005).

[5] M. Cada, M. Torrilhon, Compact third-order limiter functions for finite volume methods, Journal of Computational Physics 228 (2009) 4118-5145.

[6] P. Colella, Multidimensional upwind methods for hyperbolic copnservation laws, Journal of Computational Physics 87 (1990) 171-200.

[7] P. van Ransbeeck, C. Hirsch, New upwind dissipation models with a multidimensional approach, Aiaa paper 92-0436 (1992).

[8] D. Sidikover, Multidimensional upwinding and multigrid, Aiaa paper 951759 (1995).

[9] K. H. Kim, C. Kim, Accurate, efficient and monotonic numerical methods for multi-dimenional compressible flows, part ii: Multi-dimensional limiting process, Journal of Computational Physics 208 (2005) 570-615. 
[10] S. Kim, S. Lee, K.-H. Kim, Wavenumber-extended high-order oscillation control finite volume schemes for multi-dimensional aeroacoustic simulations, Journal of Computational Physics 227 (2008) 4089-4122.

[11] H.-M. Kang, K. H. Kim, D.-H. Lee, A new approach of a limiting process for mult-dimesnional flows, Journal of Computational Physics 229 (2010) $7102-7128$.

[12] A. Harten, B. Enquist, S. Osher, S. R. Chakravarthy, Uniform high order accurate essentially non-oscillatory schemes iii, Journal of Computational Physics 71 (1987) 231-303.

[13] C. W. Shu, S. Osher, Efficient implementation of esssentially non-oszillatory shock-capturing schemes, Journal of Computational Physics 77 (1988) 439471.

[14] X.-D. Liu, S. Osher, T. Chan, Weighted essentially non-oszillatory schemes, Journal of Computational Physics 115 (1994) 200-212.

[15] A. L. Scandaliato, M.-S. Liou, Ausm-based high-order solution for the euler equations, Aiaa paper 2010-719 (2010).

[16] M.-S. Liou, A sequel to ausm, part ii: Ausm ${ }^{+}$-up for all speeds, Journal of Computational Physics 214 (2006) 137-170.

[17] T. J. Coakley, P. G. Huang, Turbulence modeling for high speed flows, Aiaa paper 92-0436 (1992).

[18] A. Jameson, S. Yoon, Lower-upper implicit schemes with multiple grids for the euler equations, AIAA Journal 25 (7) (1987) 929-935.

[19] J. S. Shuen, Upwind differencing and lu factorization for chemical nonequilibrium navier stokes equations, Journal of Computational Physics 99 (1992) 233-250. 
[20] P. Gerlinger, P. Stoll, D. Brüggemann, An implicit multigrid method for the simulation of chemically reacting flows, Journal of Computational Physics 146 (1998) 322-345.

[21] J. S. Park, S. H. Yoon, C. Kim, Multi-dimensional limiting process for hyperbolic conservation laws on unstructured grids, Journal of Computational Physics 229 (2010) 788-812.

[22] N. W. Waterson, H. Deconinck, Design principles for bounded higher-order convection schemes - a unified approach, Journal of Computational Physics 224 (2007) 182-207.

[23] P. K. Sweby, High resolution schemes using flux limiters for hyperbolic conservation laws, SIAM Journal on Numerical Analysis 21 (5) (1984) 9951011.

[24] S. Spekreijse, Multigrid solution of monotone second-order discretizations of hyperbolic conservation laws, Mathematics of Computation 49 (179) (1987) 135-155.

[25] T. Barth, Numerical mothods for conservation laws on structured and unstructured meshes, Vki lecture series (2003).

[26] A. Jameson, W. Schmidt, E. Turkel, Numerical solution of the euler equations by finite volume methods using runge-kutta time stepping schemes, Aiaa paper 81-1259 (1981).

[27] J. McDaniels, D. Fletcher, R. H. Jr., S. Hallo, Staged transverse injection into mach 2 flow behind a rearward facing step: A 3d compressible test case for hypersonic combustion code validation, Aiaa paper 91-5071 (1991).

[28] P. Gerlinger, D. Brüggemann, An implicit multigrid scheme for the compressible navier-stokes equations with low-reynolds-number turbulence closure, Journal of Fluids Engineering 120 (1998) 257-262. 
[29] P. Gerlinger, D. Brüggemann, Numerical investigation of hydrogen strut injections into supersonic airflows, Journal of Propulsion and Power 16 (2000) 22-28.

[30] A. F. Charwat, L. G. Redekeopp, Supersonic interference flow along the corner of intersecting wedges, AIAA Journal 5 (1) (1967) 480-488.

[31] J. E. West, R. H. Korkegi, Supersonic interaction in the corner of intersecting wedges at high reynolds numbers, AIAA Journal 10 (5) (1972) 652-656.

[32] W. Marshall, S. Pal, R. Woodward, R. Santoro, Benchmark wall heat flux data for a $\mathrm{go}_{2} / \mathrm{gh}_{2}$ single element combustor, in: 41st AIAA/ASME/SAE/ASEE Joint Propulsion Conference \& Exhibit, no. 2005-3572, AIAA, 2005.

[33] M. Lempke, P. Gerlinger, M. Aigner, M. Rachner, Steady and unsteady rans simulations of cryogenic rocket combustors, Aiaa paper 2011-0101 (2011).

[34] M. Lempke, P. Gerlinger, M. Aigner, Assumed pdf modeling in rocket combustor simulations, in: Proceedings of the 4th European Conference for Aerospace Sciences, EUCASS, 2011.

[35] M.Ó Conaire, H. J. Curran, J. M. Simmie, W. J. Pitz, C. K. Westbrook, A comprehensive modeling study of hydrogen oxidation, International Journal of Chemical Kinetics 36 (11) (2004) 603-622. 


\section{Figures}

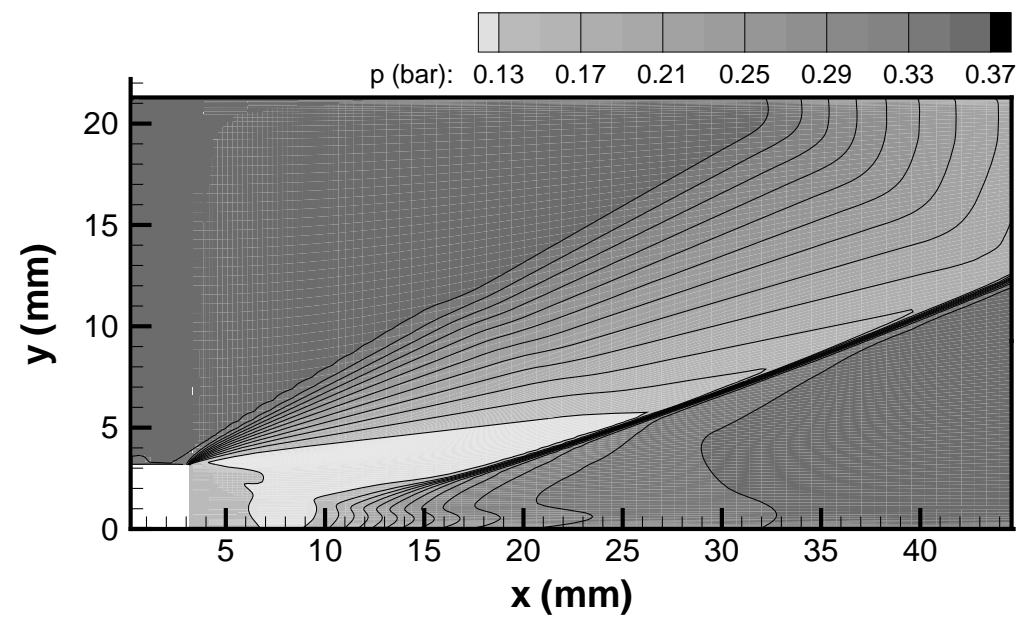

Figure 11: Calculated pressure contours for a supersonic flow over a backward facing step using the fourth order $\mathrm{MLP}^{l d}$ scheme. 

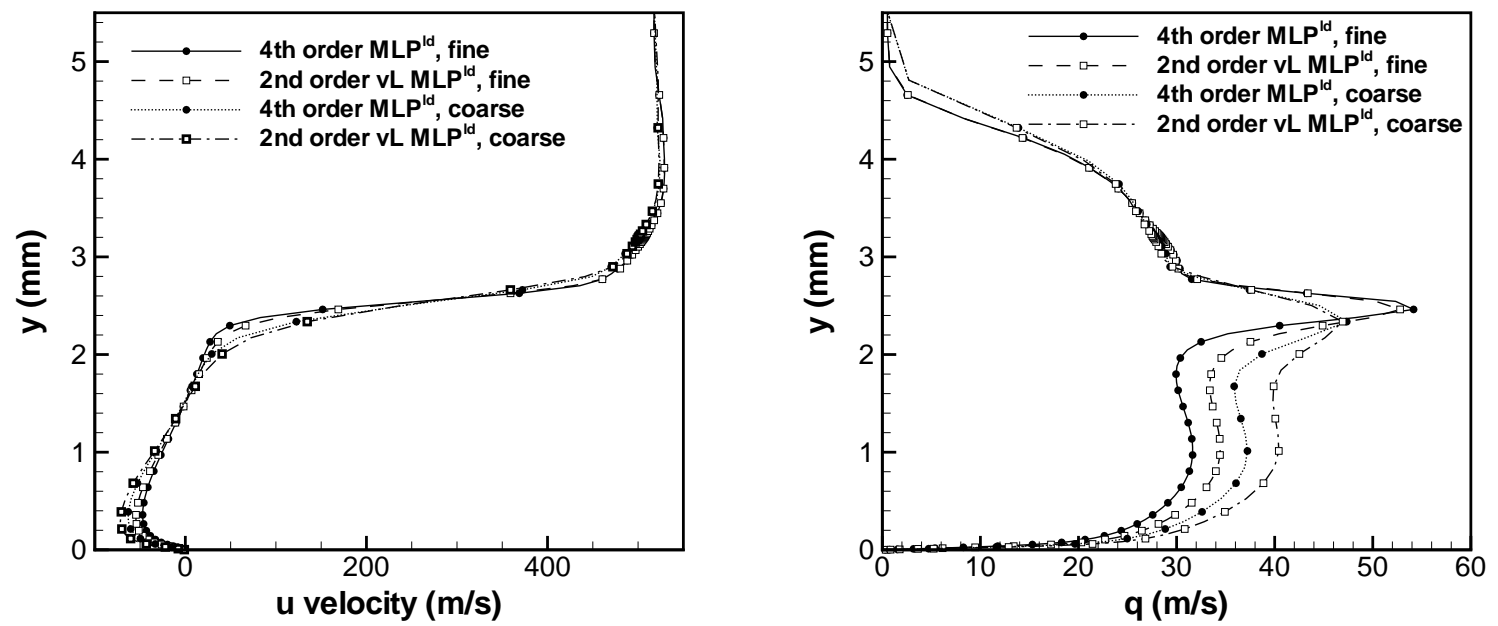

Figure 12: Vertical profiles of the velocity component $u$ (left side) and the turbulence variable $q$ (right side) at $x=5 \mathrm{~mm}$ using different discretization techniques (vL - van Leer limiter). Symbols are shown for every second grid point. 

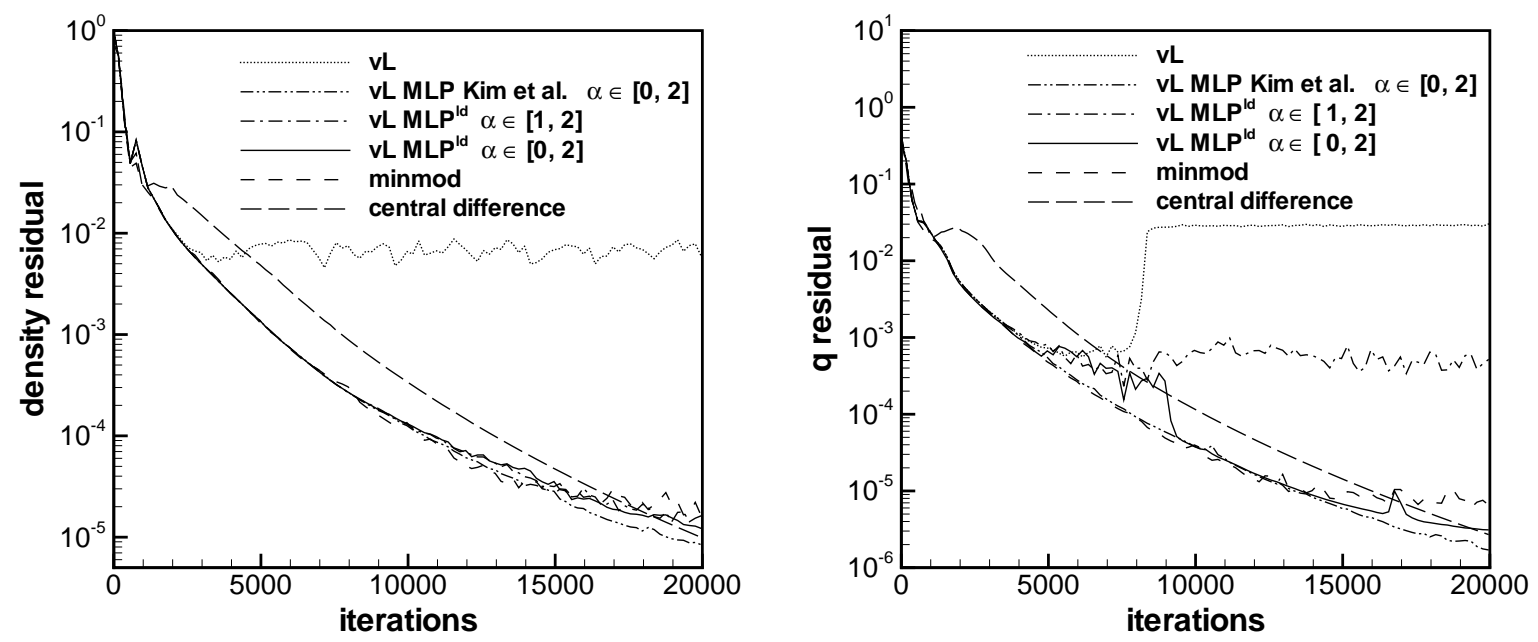

Figure 13: Convergence histories (left side density, right side turbulence residuals) for backward facing step simulations using different second order discretization techniques (vL - van Leer limiter).
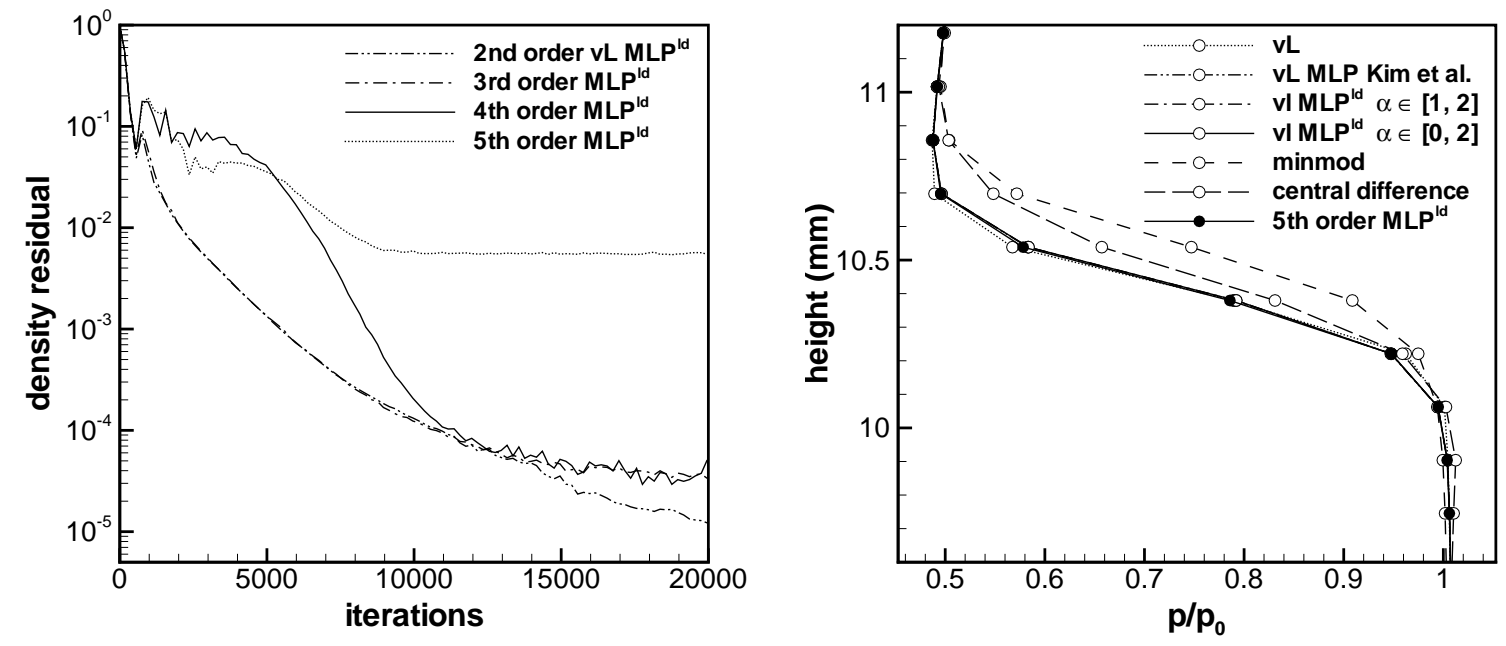

Figure 14: Convergence histories (left side) for backward facing step simulations using different higher order discretization techniques. Normalized pressure profiles (right side) across the shock wave at $x=40 \mathrm{~mm}$ versus channel height (vL - van Leer limiter). 

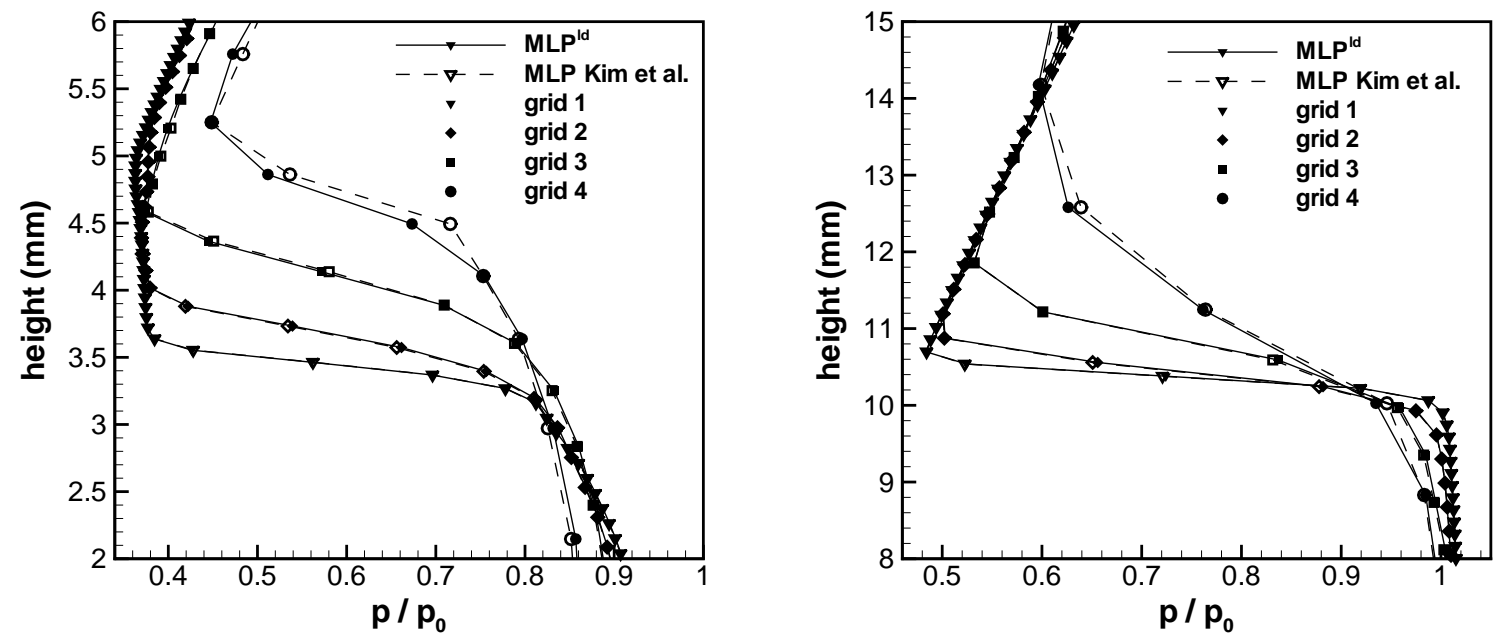

Figure 15: Normalized pressure profiles across the shock wave at $x=20 \mathrm{~mm}$ (left side) and $x=40 \mathrm{~mm}$ (right side) versus channel height. Simulations with fourth order MLP ${ }^{l d}$ and the MLP version of Kim et al. using different fine grids (grid 1 finest, grid 4 coarsest grid). 

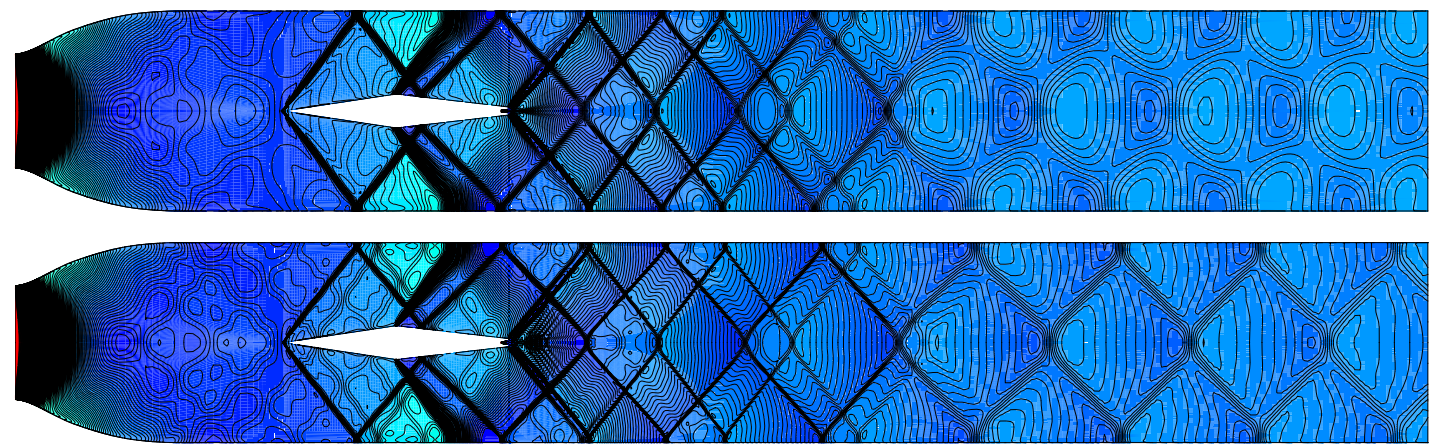

Figure 16: Pressure contours for a Mach 2 supersonic mixing channel with planar hydrogen strut injector. Results of simulations with second order central differences and artificial viscosity (top) and fourth order MLP ${ }^{l d}$ discretization (bottom). The $y$-coordinate is stretched by a factor of two.

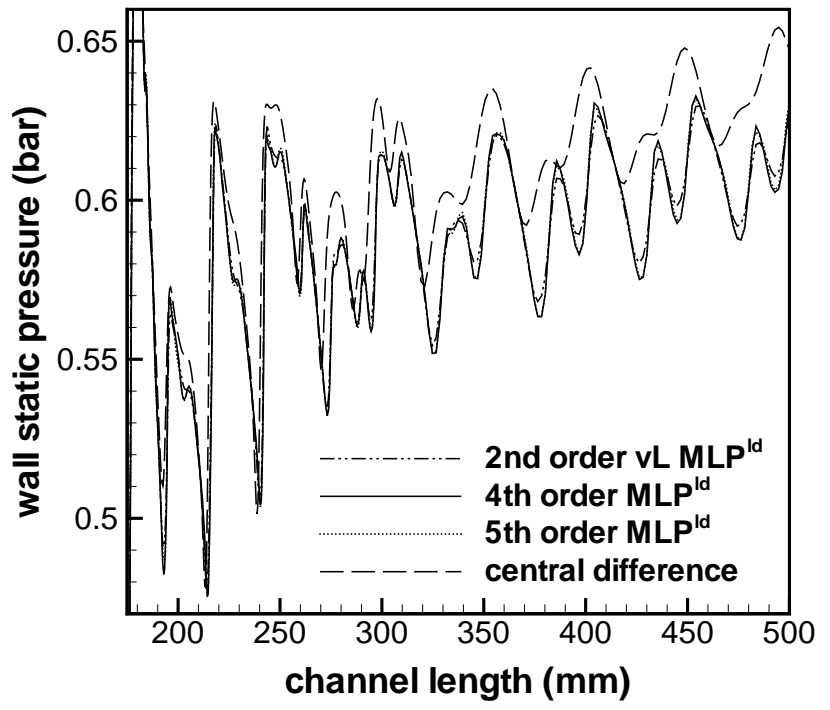

Figure 17: Wall static pressures along the channel length of a Mach 2 supersonic mixing channel. Results are compared for simulations using the second, fourth, and fifth order MLP ${ }^{l d}$ discretizations, and a second order central difference scheme. 


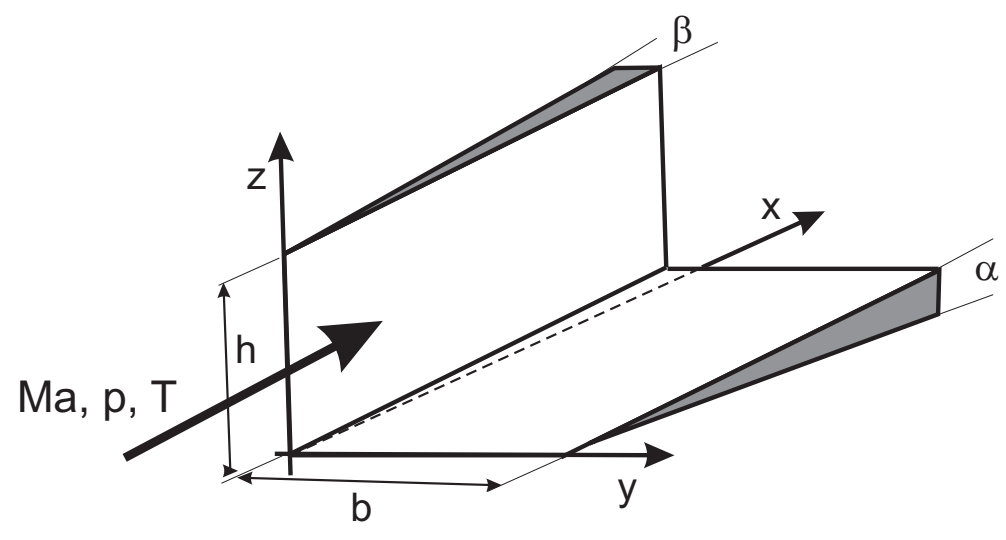

Figure 18: Sketch of the experimental setup to study the corner flow between two intersecting wedges.

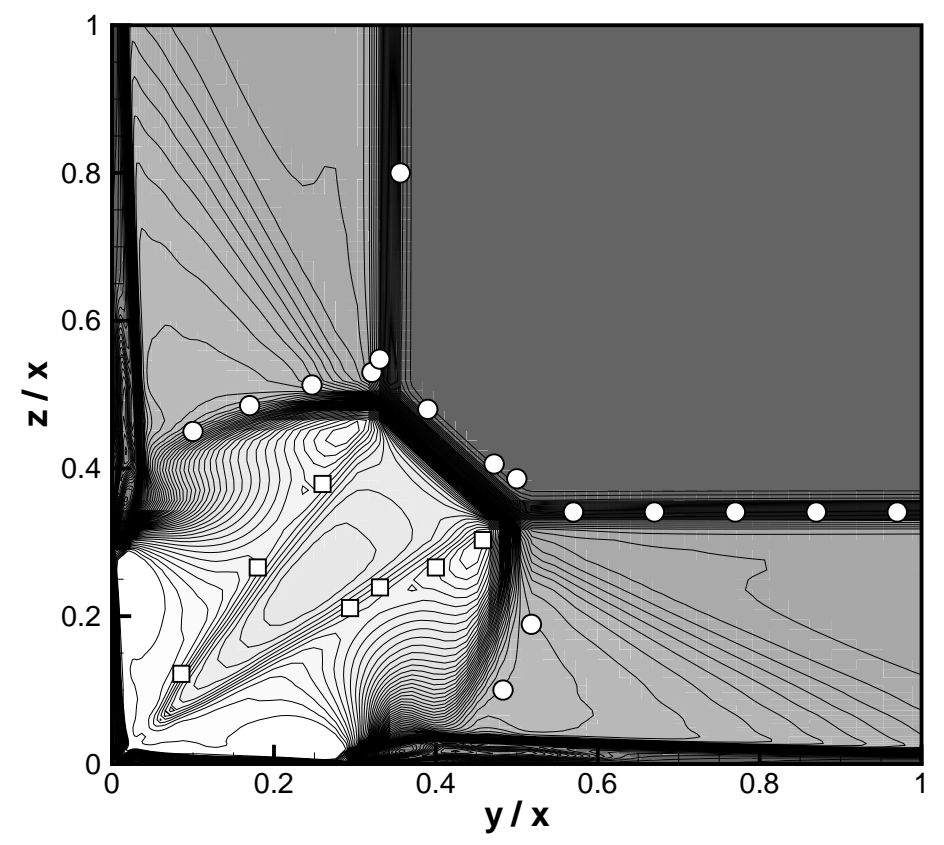

Figure 19: Calculated density contours at $x=25.4 \mathrm{~mm}$ for the laminar flow between two intersecting wedges. The simulation used the fourth order $\mathrm{MLP}^{l d}$ scheme. Symbols indicate experimentally obtained flow structures (circle 22 show the wedge shocks, the corner shock and the embedded shocks, squares slip surfaces). 


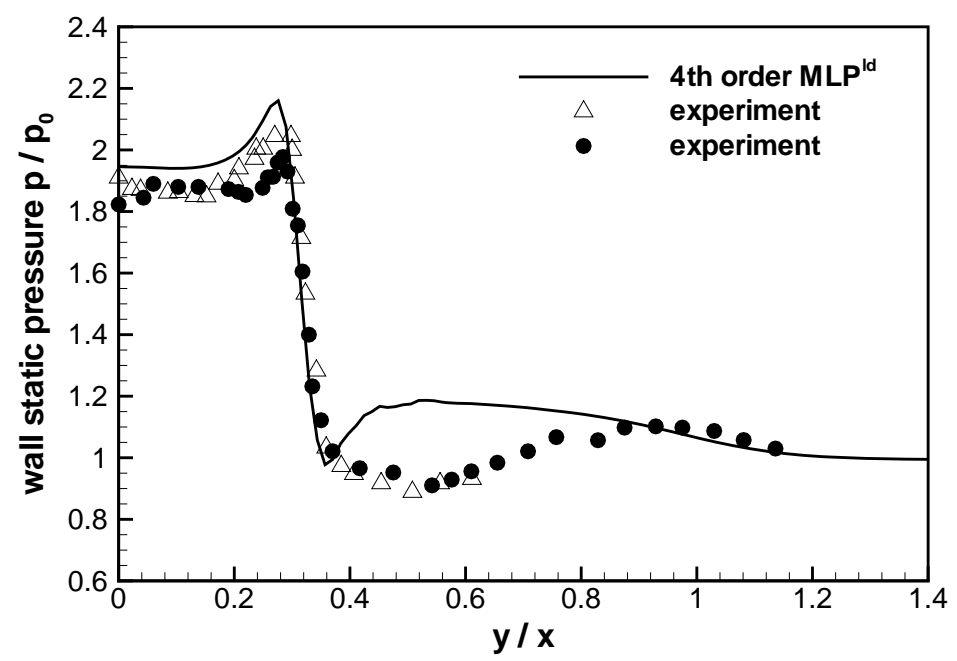

Figure 20: Experimental (two sets of measurements) and numerical wall static pressures at $x$ $=25.4 \mathrm{~mm}$ for the laminar flow between two intersecting wedges. The simulation used the fourth order $\mathrm{MLP}^{l d}$ scheme. 

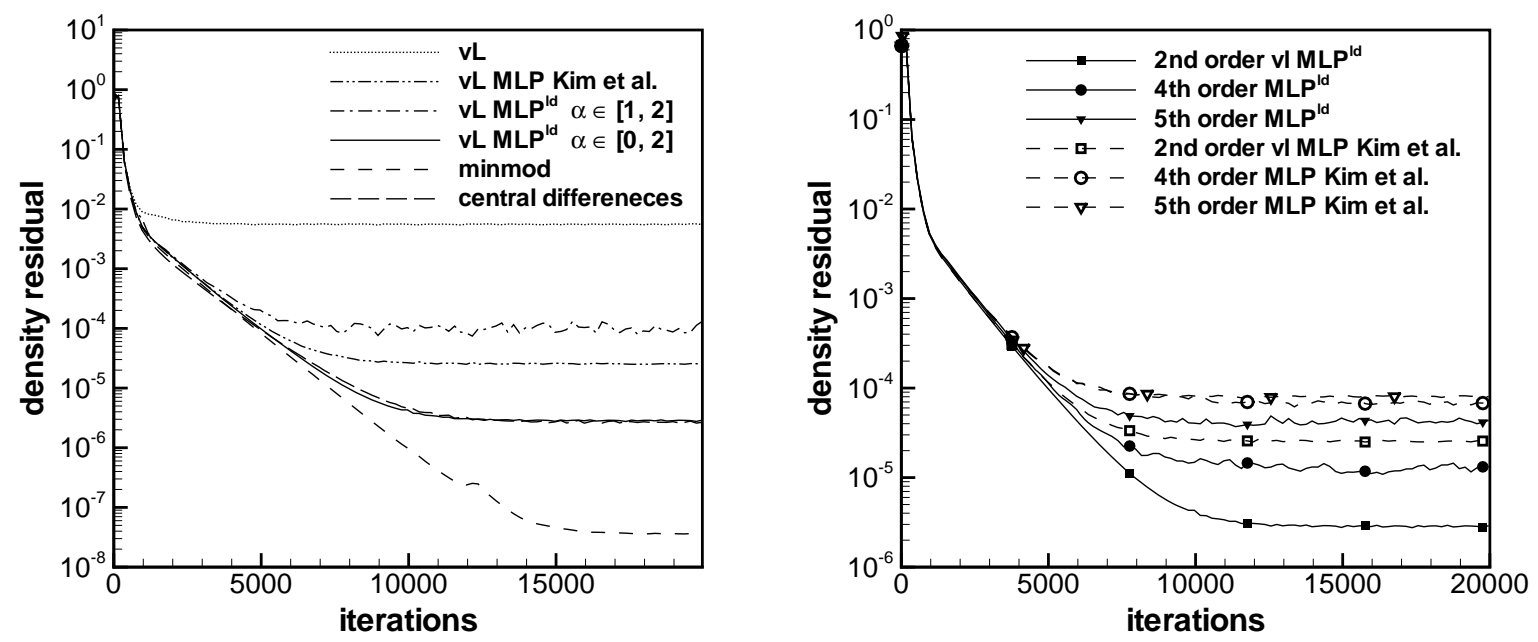

Figure 21: Convergence histories for a laminar supersonic flow over intersecting wedges. On the left side results of second order schemes, on the right side of higher order MLP schemes $\left(\mathrm{MLP}^{l d}\right.$ and MLP version of Kim et al. [1] with $\left.\alpha_{x}, \alpha_{y} \in[0,2]\right)$ are given.
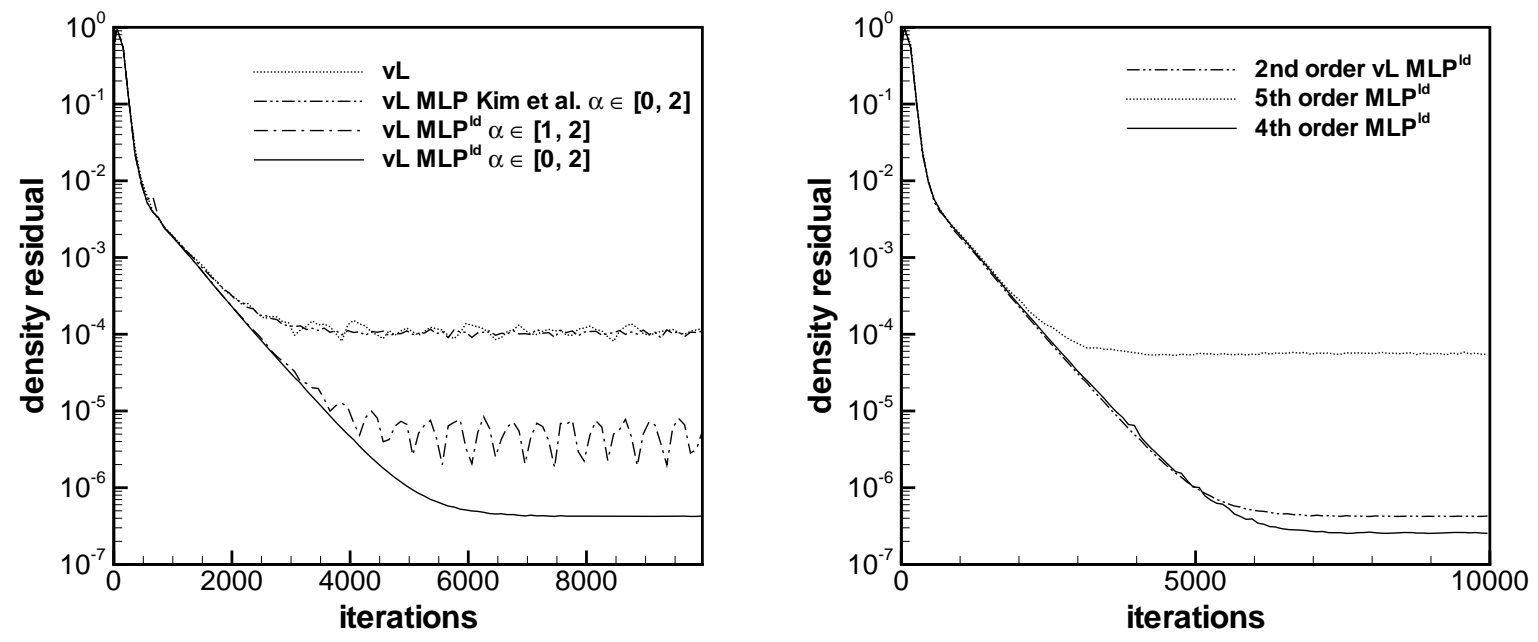

Figure 22: Convergence histories for a laminar supersonic flow over intersecting wedges using a coarse computational grid. On the left side results of second order schemes, on the right side of higher order $\mathrm{MLP}^{l d}$ schemes are given. 

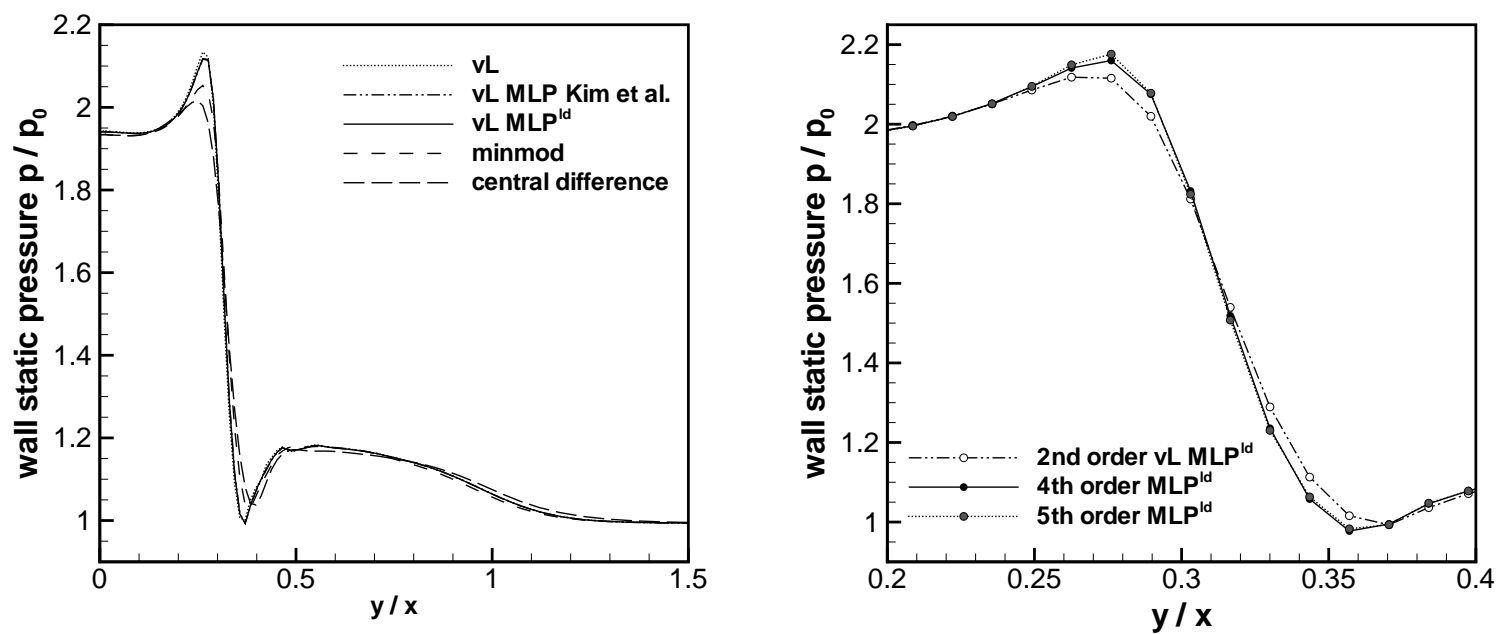

Figure 23: Wall static pressures at $x=25.4 \mathrm{~mm}$ for a laminar flow over two intersecting wedges. The simulations used different second order discretizations (left side) and higher order $\mathrm{MLP}^{l d}$ discretizations (right side). On the right side an extension of the shock region is shown.
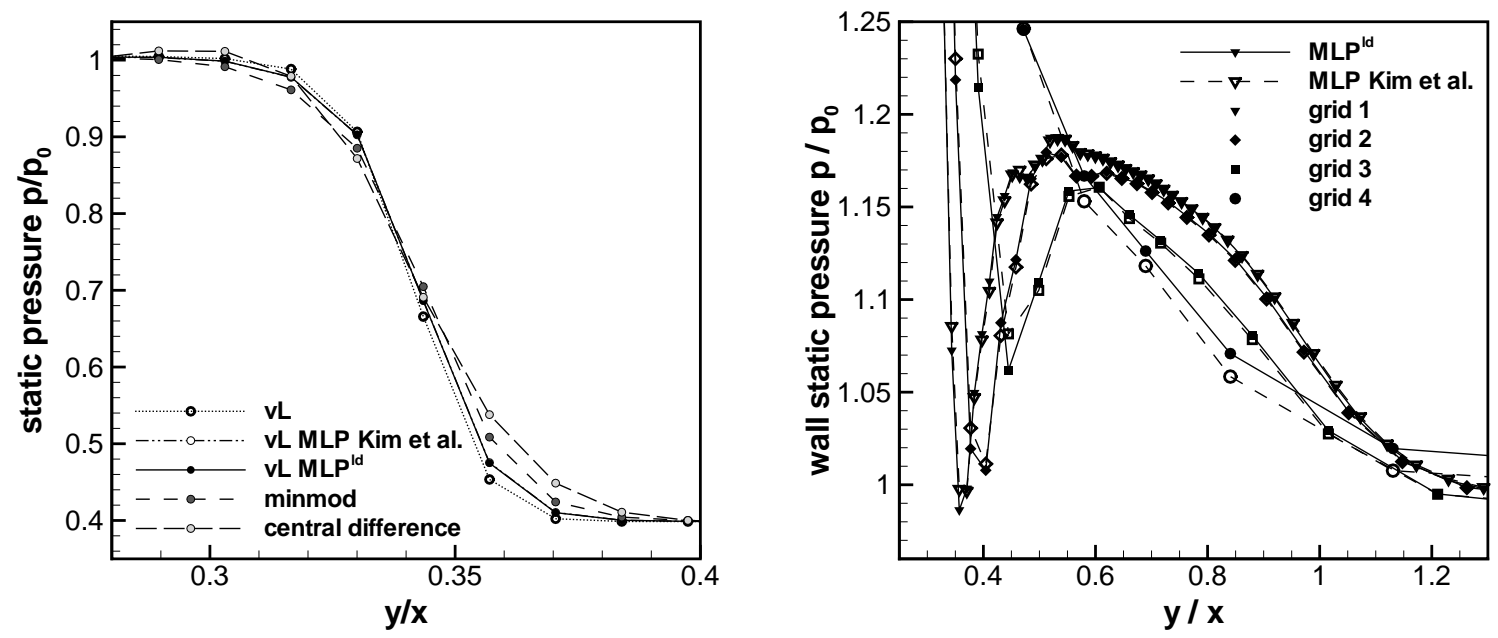

Figure 24: Laminar flow over two intersecting wedges. Left side: Static pressures at $x=25.4$ $\mathrm{mm}$ and $z=58.1 \mathrm{~mm}$. The simulations used different second order discretizations. Right side: Wall static pressures at $x=25.4 \mathrm{~mm}$ for simulations with the fifth order MLP ${ }^{l d}$ scheme and the MLP version of Kim et al. on different fine grids (grid 1 finest, grid 4 coarsest grid). 


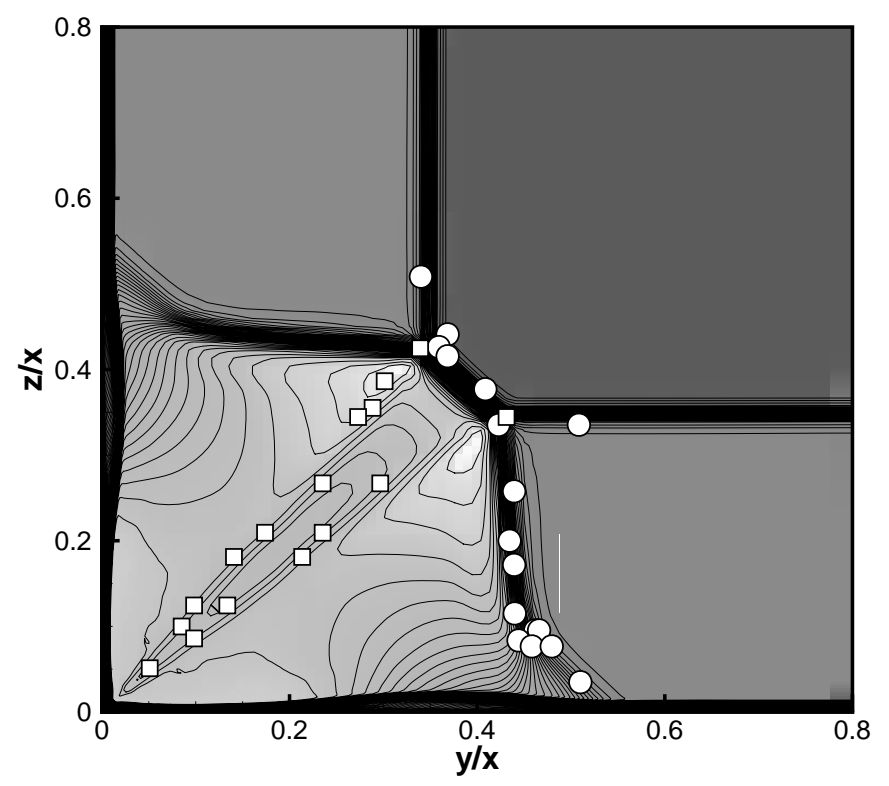

Figure 25: Calculated density contours at $x=87.6 \mathrm{~mm}$ for a turbulent flow over two intersecting wedges. The simulation used the fourth order MLP ${ }^{l d}$ scheme. Symbols indicate experimentally obtained flow structures (circles show the wedge shocks, corner shock, and the embedded shocks, squares slip surfaces).

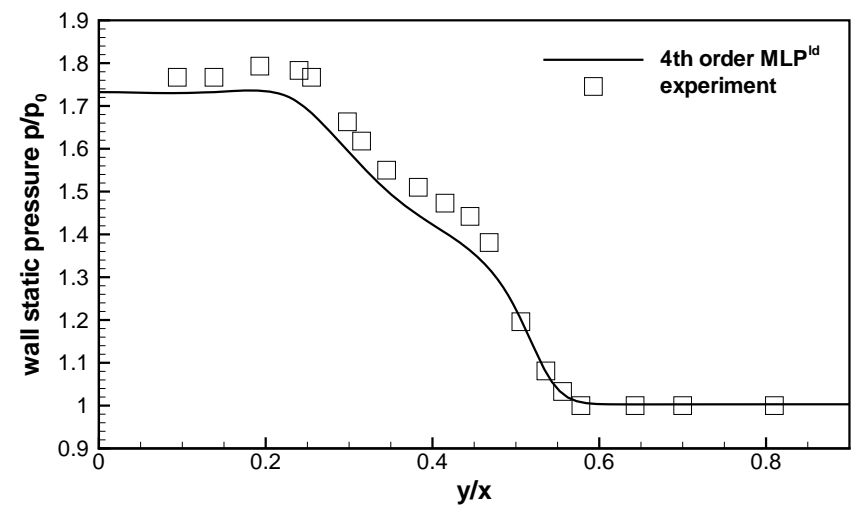

Figure 26: Experimental and numerical wall static pressures at $x=87.6 \mathrm{~mm}$ for a turbulent flow over two intersecting wedges. The simulation used the fourth order $\mathrm{MLP}^{l d}$ scheme. 

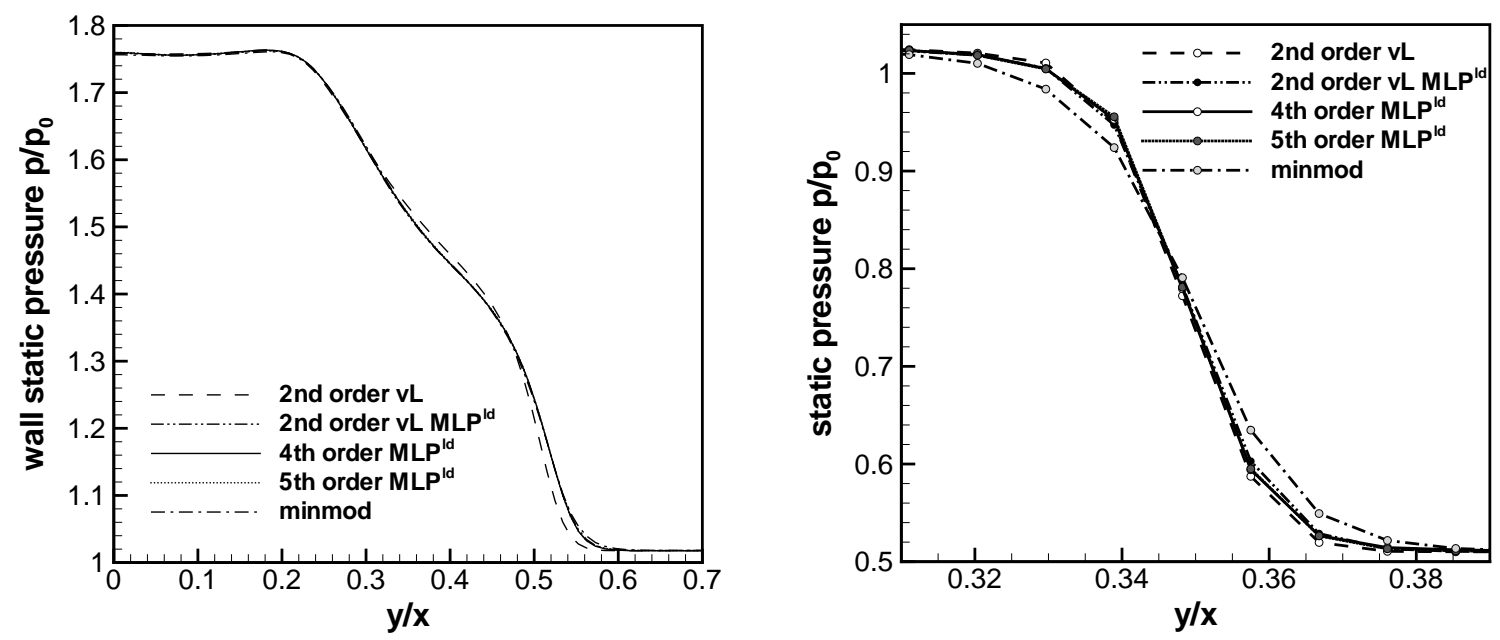

Figure 27: Wall static pressures (left side) at $x=87.6 \mathrm{~mm}$ for a turbulent flow over two intersecting wedges using different discretization techniques. Static pressures at $x=87.6 \mathrm{~mm}$ and $z=148 \mathrm{~mm}$ (right side) using different discretization techniques (extension of the shock region).
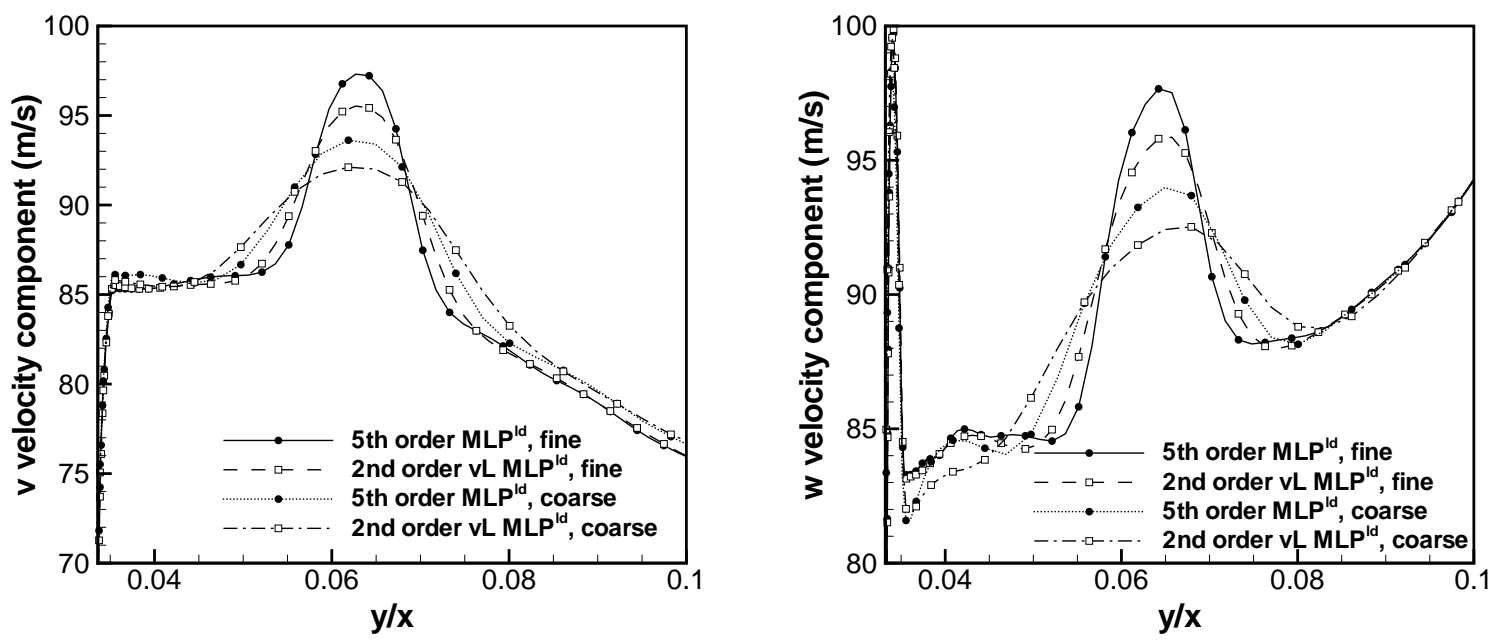

Figure 28: Profiles of the velocity components $v$ (left side) and $w$ (right side) in dependence of the $y$-coordinate at $x=100 \mathrm{~mm}$ and $h=15 \mathrm{~mm}$ above the lower wall. The simulations use different discretization techniques (vL - van Leer limiter). Symbols are shown for every second grid point. 

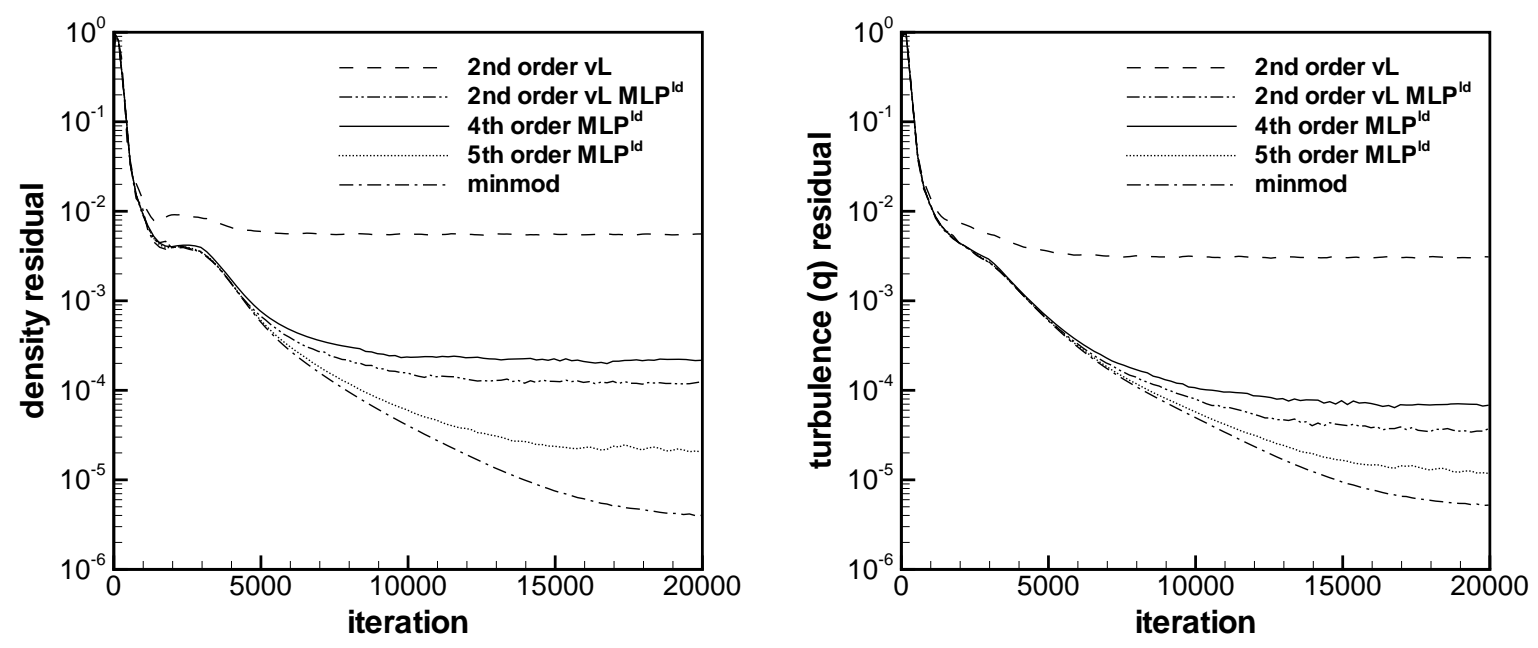

Figure 29: Convergence histories (left side density, right side turbulence $q$ residual) for a turbulent flow over two intersecting wedges.

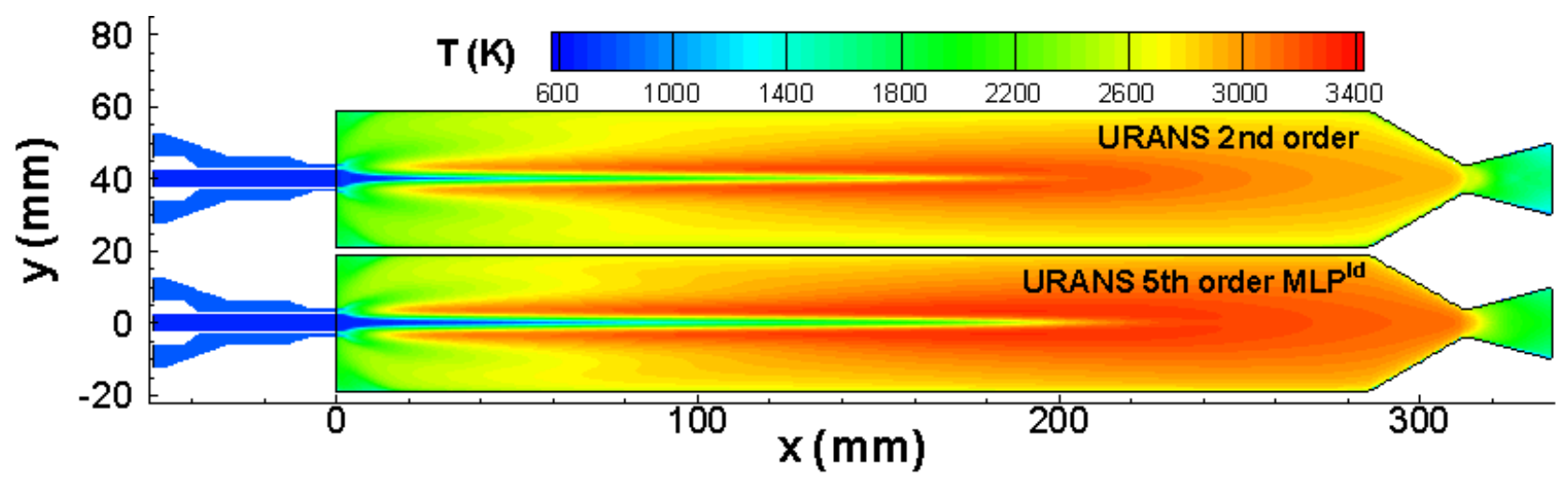

Figure 30: Calculated averaged temperature distributions for the PennState model rocket combustor [32]. In the upper figure a second order discretization with van Albada limiter is used, the lower figure is based on the fifth order $\operatorname{MLP}^{l d}$ scheme. 


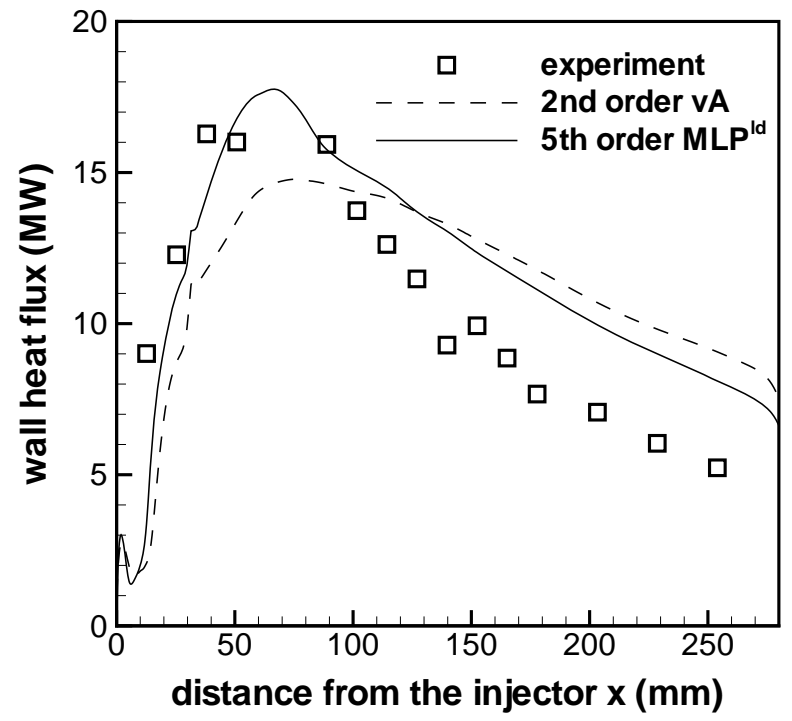

Figure 31: Experimentally measured [32] and simulated wall heat fluxes of the PennState model rocket combustor. Numerical results are for a second order scheme with van Albada limiter and a fifth order $\mathrm{MLP}^{l d}$ scheme. 